\title{
Catalytic Asymmetric Assembly of Stereodefined Propionate Units: An Enantioselective Total Synthesis of (-)-Pironetin
}

\author{
Supporting Information
}

\author{
Xiaoqiang Shen, Andrew S. Wasmuth, Junping Zhao, Cheng Zhu, and Scott G. Nelson* \\ Department of Chemistry, University of Pittsburgh, Pittsburgh, Pennsylvania 15260
}

TABLE OF CONTENTS

Compounds 8-11, 13a, 13b, 14a, 14c, 16, 18, 19-26, 28, 29

page S1-S10

Stereochemical proofs (X-ray data) for compounds $14 \mathrm{c}$ and 16

page $\mathrm{S} 11-\mathrm{S} 12$

${ }^{1} \mathrm{H}$ and ${ }^{13} \mathrm{C}$ Spectra for compounds 8, 9-11, 13a, 13b, 14a, 14c, 16, 18, 19-26, 28, 29

page S13-S60

General Information: Unless otherwise stated, all reactions were carried out in dry glassware under a nitrogen atmosphere using standard inert atmosphere $\left(\mathrm{N}_{2}\right)$ techniques for the manipulation of solvents and reagents. Proton NMR chemical shifts are reported relative to residual $\mathrm{CHCl}_{3}(7.27 \mathrm{ppm})$ for ${ }^{1} \mathrm{H}$ and $\mathrm{CDCl}_{3}(77.00 \mathrm{ppm})$ for ${ }^{13} \mathrm{C}$ NMR spectra. Analytical high performance liquid chromatography (HPLC) was performed using a variable wavelength UV detector (deuterium lamp, 190-600 nm) using a Daicel Chiracel ${ }^{\mathrm{TM}}$ OD-H column $(250$ $\mathrm{x} 4.6 \mathrm{~mm}$ ) (Daicel Inc.) and HPLC-grade isopropanol and hexanes as the eluting solvents. Anhydrous solvents $\left(\mathrm{CH}_{2} \mathrm{Cl}_{2}\right.$, THF, diethyl ether, pentane, $N, N$-dimethylformamide and toluene) were obtained by passage through successive alumina- and Q5 reactant-packed columns on a solvent purification system. N, NDiisopropylethylamine and triethylamine were distilled under nitrogen from $\mathrm{CaH}_{2}$. The enantioenriched aldehydes 12a-c and 17 were prepared from the corresponding $\beta$-lactones using the three-step sequence described for converting $\beta$-lactone 8 to aldehyde 9. ${ }^{1,2}$ Aldehydes 12a-c, 15, and $\mathbf{1 7}$ were prepared with the indicated stereochemical purities: 12a (99\% ee), 12b (99\% ee), 12c $\left(99 \%\right.$ ee), $15\left(95 \%\right.$ ee), $17\left(>97 \%\right.$ de).$^{3}$ Catalyst 27 was prepared according to the literature procedure. ${ }^{4}$ Anhydrous $\mathrm{LiClO}_{4}$ and LiI were purchased from Aldrich Chemical Co. and weighed out in a $\mathrm{N}_{2}$-filled glovebox. O-Trimethylsilylquinidine (3a, TMSQd) ${ }^{5}$ and $O$-trimethylsilylquinine (4a, TMSQn), ${ }^{5}\left[\left({ }^{c} \mathrm{C}_{8} \mathrm{H}_{14}\right)_{2} \mathrm{IrCl}\right]_{2}{ }^{6}$ and 3 -p-methoxybenzyloxypropanal ${ }^{7}$ were prepared according to literature procedures. Commercially available propionyl chloride was redistilled under $\mathrm{N}_{2}$. Butyryl bromide was prepared according to literature procedure and was distilled from $\mathrm{P}_{2} \mathrm{O}_{5}{ }^{8}$ Flash chromatography was performed as previously described on EM silica gel 60 (230-240 mesh). ${ }^{9}$

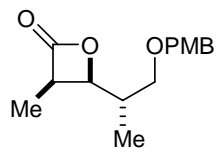

$(3 R, 4 S)-4-[(S)-1-(4-M e t h o x y b e n z y l o x y) p r o p a n-2-y l]-3-m e t h y l o x e t a n-2-o n e ~(8):$ To a -78 ${ }^{\circ} \mathrm{C}$ solution of $O$-trimehtylsilylquinine (4a) $(40 \mathrm{mg}, 0.1 \mathrm{mmol})$ and $\mathrm{LiClO}_{4}(108 \mathrm{mg}, 1.0 \mathrm{mmol})$ in $2: 1 \mathrm{CH}_{2} \mathrm{Cl}_{2}: \mathrm{Et}_{2} \mathrm{O}(3 \mathrm{~mL})$ was added $N, N$-diisopropylethylamine $(0.44 \mathrm{~mL}, 2.5 \mathrm{mmol})$ and (S)-3-(4-methoxybenzyloxy)-2-methylpropanal $(210 \mathrm{mg}, 1.0 \mathrm{mmol})$. To the resulting mixture

\footnotetext{
${ }^{1}$ Nelson, S. G.; Cheung, W. S.; Kassick, A. J.; Hilfiker, M. A. J. Am. Chem. Soc. 2002, 124, 13654.

${ }^{2}$ For aldehyde 12c, the $p$-methoxybenzyl ether was introduced according to the published procedure: Clark, D. L.; Heathcock, C. H. J. Org. Chem. 1993, 58, 5878.

${ }^{3}$ Optical purities for aldehydes $\mathbf{1 2 a}$-c and $\mathbf{1 5}$ are based on those for the $\beta$-lactone precursors assayed by chiral GC, see: Zhu, C.; Shen, X.; Nelson, S. G. J. Am. Chem. Soc. 2004, 126, 5352. The stereochemical purity of aldehyde 17 was assayed by integrating the appropriate resonances in the $500 \mathrm{MHz}{ }^{1} \mathrm{H}$ NMR.

${ }^{4}$ Nelson, S. G.; Zhu, C.; Shen, X. J. Am. Chem. Soc. 2004, 126, 14.

${ }^{5}$ Calter, M. A. J. Org. Chem. 1996, 61, 8006.

${ }^{6}$ Onderdelinden, A. L.; Van der Ent, A. Inorg. Chim. Acta 1972, 6, 420.

${ }^{7}$ Zampella, A.; Sepe, V.; D’Orsi, R.; Bifulco, G.; Bassarello, C.; D'Auria, V. Tetrahedron Asymm. 2003, $14,1787$.

${ }^{8}$ Burton, T. M.; Degering, E. F. J. Am. Chem. Soc. 1940, 62, 227.

${ }^{9}$ Still, W. C.; Kahn, M.; Mitra, A. J. Org. Chem. 1978, 43, 2923.
} 
was added a solution of propionyl chloride $(0.17 \mathrm{~mL}, 2.0 \mathrm{mmol})$ in $0.5 \mathrm{~mL} \mathrm{CH}_{2} \mathrm{Cl}_{2}$ over $2 \mathrm{~h}$ by syringe pump. The reaction was stirred $16 \mathrm{~h}$ at $-78{ }^{\circ} \mathrm{C}$ whereupon $\mathrm{Et}_{2} \mathrm{O}(10 \mathrm{~mL})$ was added and the reaction was warmed to ambient temperature. The resulting mixture was filtered through a silica gel plug eluting with $\mathrm{Et}_{2} \mathrm{O}(100 \mathrm{~mL})$. ${ }^{1} \mathrm{H}$ NMR analysis of the crude product revealed the diastereomer ratio to be $92: 8$ syn, anti: syn:syn. The filtrate was concentrated in vacuo and the crude product mixture was purified by flash chromatography $\left(\mathrm{SiO}_{2}, 21 \%\right.$ ethyl acetate in hexanes) giving $195 \mathrm{mg}(74 \%)$ of the title compound as light yellow oil. $[\alpha]_{\mathrm{D}}-29(c 0.31$, $\mathrm{CHCl}_{3}$ ); IR (thin film): 1823, 1613, 1513, 1463, 1247, 1117, $986 \mathrm{~cm}^{-1} ;{ }^{1} \mathrm{H}$ NMR $\left(300 \mathrm{MHz}, \mathrm{CDCl}_{3}\right): \delta 1.09(\mathrm{~d}$, $\mathrm{J}=6.7 \mathrm{~Hz}, 3 \mathrm{H}), 1.42(\mathrm{~d}, \mathrm{~J}=7.7 \mathrm{~Hz}, 3 \mathrm{H}), 2.13-2.21(\mathrm{~m}, 1 \mathrm{H}), 3.56(\mathrm{dd}, \mathrm{J}=9.0,5.7 \mathrm{~Hz}, 1 \mathrm{H}), 3.64$ (dd, J=9.0, 3.1 Hz, 1H), 3.80-3.89 (m, 1H), 3.89 (s, 3H), 4.51 (dd, J=11.0, $6.3 \mathrm{~Hz}, 1 \mathrm{H}), 4.54(\mathrm{~s}, 2 \mathrm{H}), 6.96(\mathrm{~d}, \mathrm{~J}=8.5 \mathrm{~Hz}, 2 \mathrm{H}), 7.53$ $(\mathrm{d}, \mathrm{J}=8.4 \mathrm{~Hz}, 2 \mathrm{H}) ;{ }^{13} \mathrm{C} \mathrm{NMR}\left(75 \mathrm{MHz}, \mathrm{CDCl}_{3}\right): \delta 8.4,13.0,34.2,46.9,55.2,70.8,72.9,76.2,113.7,129.1$, 130.3, 159.1, 172.4; MS (EI, $70 \mathrm{eV}): \mathrm{m} / \mathrm{z}$ 264, 152, 137, 121, 78; HRMS m/z calcd for $\mathrm{C}_{15} \mathrm{H}_{20} \mathrm{O}_{4}\left(\mathrm{M}^{+}\right): 264.1362$; found: 264.1360 .

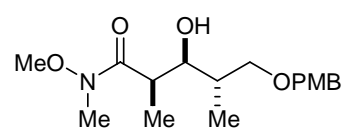

(2R, 3S, 4S)- $N$-Methoxy- $N$-methyl-3-hydroxy-5-(4-methoxybenzyloxy)- 2, 4-dimethylpentanamide (8'): To a $0{ }^{\circ} \mathrm{C}$ solution of $N$, $O$-dimethylhydroxylaamine hydrochloride $(1.64 \mathrm{~g}, 16.8 \mathrm{mmol})$ in $30 \mathrm{~mL}$ of $\mathrm{CH}_{2} \mathrm{Cl}_{2}$ was added $\mathrm{Me}_{2} \mathrm{AlCl}(1.0 \mathrm{M}$ in hexane, $16.8 \mathrm{~mL}, 16.8 \mathrm{mmol}$ ) added dropwise. Once addition was complete, the reaction was warmed to ambient temperature and stirred for $60 \mathrm{~min}$. The reaction ws cooled to $-30{ }^{\circ} \mathrm{C}$ and a solution of lactone $7(2.23$ $\mathrm{g}, 8.40 \mathrm{mmol}$ ) in $10 \mathrm{~mL}$ of $\mathrm{CH}_{2} \mathrm{Cl}_{2}$ was added over $60 \mathrm{~min}$ via syringe pump. Once addition was complete, the reaction was stirred for $60 \mathrm{~min}$ then $100 \mathrm{~mL}$ of saturated aqueous sodium tartrate was added. The resulting gel like mixture was warmed to ambient temperature and stirred vigorously until two homogeneous phases resulted. The mixture was diluted with $100 \mathrm{~mL}$ of $\mathrm{CH}_{2} \mathrm{Cl}_{2}$ and the layers were shaken and separated. The aqueous portion was extracted with $\mathrm{CH}_{2} \mathrm{Cl}_{2}(3 \times 50 \mathrm{~mL})$ and the combined organic extracts were dried $\left(\mathrm{Na}_{2} \mathrm{SO}_{4}\right)$ and concentrated. The crude product mixture was purified by column chromatography $\left(\mathrm{SiO}_{2}\right.$, gradient $10 \rightarrow 50 \%$ EtOAc in hexanes) to afford $2.66 \mathrm{~g}(80 \%)$ of the title compound as colorless oil. $[\alpha]_{\mathrm{D}}=-13\left(c 1.8, \mathrm{CHCl}_{3}\right)$; IR $v$ (thin film) $3458,1636,1586,1513,1463,1247,1032,820 \mathrm{~cm}^{-1} ;{ }^{1} \mathrm{H}$ NMR $\left(300 \mathrm{MHz}, \mathrm{CDCl}_{3}\right): \delta 0.98(\mathrm{~d}, \mathrm{~J}=6.9,3 \mathrm{H})$, $1.18(\mathrm{~d}, \mathrm{~J}=7.0 \mathrm{~Hz}, 3 \mathrm{H}), 1.82-1.94(\mathrm{~m}, 1 \mathrm{H}), 3.04($ broad s, 1H), $3.19(\mathrm{~s}, 3 \mathrm{H}), 3.53(\mathrm{dd}, \mathrm{J}=9.0,5.8 \mathrm{~Hz}, 1 \mathrm{H}), 3.63$ (dd, J=9.1, $4.0 \mathrm{~Hz}, 1 \mathrm{H}), 3.67$ (s, 3H), 3.68-3.73(m, 2H), $3.80(\mathrm{~S}, 3 \mathrm{H}), 4.45(\mathrm{~s}, 2 \mathrm{H}), 6.87(\mathrm{~d}, \mathrm{~J}=8.4 \mathrm{~Hz}, 2 \mathrm{H}), 7.26$ $(\mathrm{d}, \mathrm{J}=8.4 \mathrm{~Hz}, 2 \mathrm{H}) ;{ }^{13} \mathrm{C} \mathrm{NMR}\left(75 \mathrm{MHz}, \mathrm{CDCl}_{3}\right): \delta 10.4,14.2,32.0,36.0,36.4,55.2,61.4,72.7,72.9,73.8,113.7$, 129.2, 130.6, 159.0, 176.6; MS (ESI): $\mathrm{m} / \mathrm{z} 348\left(\mathrm{M}^{+}+\mathrm{Na}\right)$; HRMS $\mathrm{m} / \mathrm{z}$ calcd for $\mathrm{C}_{17} \mathrm{H}_{27} \mathrm{NO}_{5} \mathrm{Na}\left(\mathrm{M}^{+}+\mathrm{Na}\right)$ : 348.1787; found: 348.1777 .

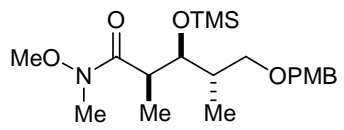

(0.47 mL, $2.6 \mathrm{mmol})$.
(2R, 3S, 4S)-N-Methoxy- $N$-methyl-5-(4-methoxybenzyloxy)-3-trimethylsiloxy-2, 4dimethylpentanamide (8"). To a $-78{ }^{\circ} \mathrm{C}$ solution of alcohol $\mathbf{8}^{\prime}(665 \mathrm{mg}, 2.00 \mathrm{mmol})$ in $18 \mathrm{~mL}$ of $\mathrm{CH}_{2} \mathrm{Cl}_{2}$ containing 2,6-lutidine $(0.35 \mathrm{~mL}, 3.0 \mathrm{mmol})$ was added TMSOTf additional $2.5 \mathrm{~h}$. The reaction was quenched by adding aqueous $1 \mathrm{~N} \mathrm{NaHSO}_{4}(40 \mathrm{~mL})$ and the resulting mixture was extracted with $\mathrm{CH}_{2} \mathrm{Cl}_{2}(50 \mathrm{~mL})$. The aqueous portion was extracted with $\mathrm{CH}_{2} \mathrm{Cl}_{2} .(2 \times 30 \mathrm{~mL})$ and the combined organic extracts dried $\left(\mathrm{Na}_{2} \mathrm{SO}_{4}\right)$ and concentrated. The crude product mixture was purified by column chromatography $\left(\mathrm{SiO}_{2}, 20 \%\right.$ EtOAc in hexanes) to afford $689 \mathrm{mg}(86 \%)$ of the title compound as a colorless oil. $[\alpha]_{\mathrm{D}}-11\left(c\right.$ 1.2, $\left.\mathrm{CHCl}_{3}\right)$; IR v (thin film) 1660, 1613, 1513, 1461, 1249, 1082, $839 \mathrm{~cm}^{-1} ;{ }^{1} \mathrm{H}$ NMR $(300 \mathrm{MHz}$, $\left.\mathrm{CDCl}_{3}\right): \delta 0.07(\mathrm{~s}, 9 \mathrm{H}), 0.95(\mathrm{~d}, \mathrm{~J}=6.9 \mathrm{~Hz}, 3 \mathrm{H}), 1.06(\mathrm{~d}, \mathrm{~J}=6.9 \mathrm{~Hz}, 3 \mathrm{H}), 1.79-1.90(\mathrm{~m}, 1 \mathrm{H}), 3.08(\mathrm{~s}, 3 \mathrm{H}), 3.08-$ $3.18(\mathrm{~m}, 2 \mathrm{H}), 3.53(\mathrm{~s}, 3 \mathrm{H}), 3.53-3.57(\mathrm{~m}, 1 \mathrm{H}), 3.84(\mathrm{dd}, \mathrm{J}=7.8,3.5 \mathrm{~Hz}, 1 \mathrm{H}), 4.35(\mathrm{~s}, 2 \mathrm{H}), 6.81(\mathrm{~d}, \mathrm{~J}=8.6 \mathrm{~Hz}, 2 \mathrm{H})$, $7.19(\mathrm{~d}, \mathrm{~J}=8.6 \mathrm{~Hz}, 2 \mathrm{H}) ;{ }^{13} \mathrm{C} \mathrm{NMR}\left(75 \mathrm{MHz}, \mathrm{CDCl}_{3}\right): \delta 0.7,14.3,16.0,32.2,37.8,39.4,55.3,61.3,71.8,72.6$, 77.1, 113.7, 129.2, 131.0, 159.0, 176.6; MS (ESI): $\mathrm{m} / \mathrm{z} 420\left(\mathrm{M}^{+}+\mathrm{Na}\right)$; HRMS m/z calcd for $\mathrm{C}_{20} \mathrm{H}_{35} \mathrm{NO}_{5} \mathrm{NaSi}$ $\left(\mathrm{M}^{+}+\mathrm{Na}\right)$ : 420.2182; found: 420.2172 .

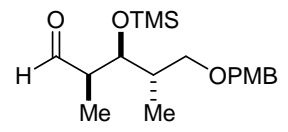

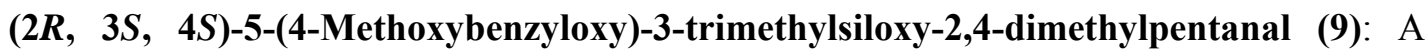
hexanes solution of ${ }^{i} \mathrm{Bu}_{2} \mathrm{AlH}(1.0 \mathrm{M}, 2 \mathrm{~mL}, 2.0 \mathrm{mmol})$ was added to a $-78{ }^{\circ} \mathrm{C}$ solution of Weinreb amide 8" (588 mg, $1.5 \mathrm{mmol})$ in $10 \mathrm{~mL}$ THF. After being stirred $3 \mathrm{~h}$, the reaction was quenched at $-78{ }^{\circ} \mathrm{C}$ by adding $40 \mathrm{~mL}$ of an aqueous, saturated sodium tartrate solution. The reaction was warmed to ambient temperature affording a gel-like mixture that was stirred until two homogeneous layers resulted $(\sim 30 \mathrm{~min})$. The mixture was diluted with $\mathrm{Et}_{2} \mathrm{O}(50 \mathrm{~mL})$ and the layers were separated. The aqueous 
portion was extracted with $\mathrm{Et}_{2} \mathrm{O}(3 \times 20 \mathrm{~mL})$ and the combined organic portions were dried and concentrated. The crude product mixture was purified by flash chromatography $\left(\mathrm{SiO}_{2}, 10 \%\right.$ ethyl acetate in hexane) giving $438 \mathrm{mg}(80 \%)$ of the title compound as colorless oil. $[\alpha]_{\mathrm{D}}-32\left(c 0.34, \mathrm{CHCl}_{3}\right)$; IR (thin film): 1725, 1613, 1513, 1463, 1249, 1032, $841 \mathrm{~cm}^{-1}$; ${ }^{1} \mathrm{H}$ NMR $\left(300 \mathrm{MHz}, \mathrm{CDCl}_{3}\right): \delta 0.07(\mathrm{~s}, 9 \mathrm{H}), 0.96(\mathrm{~d}, \mathrm{~J}=6.9 \mathrm{~Hz}, 3 \mathrm{H}), 1.08(\mathrm{~d}, \mathrm{~J}=7.0$ $\mathrm{Hz}, 3 \mathrm{H}), 1.87-1.95(\mathrm{~m}, 1 \mathrm{H}), 2.52(\mathrm{qd}, \mathrm{J}=6.9,3.4 \mathrm{~Hz}, 1 \mathrm{H}), 3.37(\mathrm{dd}, \mathrm{J}=9.0,5.7 \mathrm{~Hz}, 1 \mathrm{H}), 3.46(\mathrm{dd}, \mathrm{J}=9.0,4.5 \mathrm{~Hz}$, $1 \mathrm{H}), 3.81(\mathrm{~s}, 3 \mathrm{H}), 4.16(\mathrm{dd}, \mathrm{J}=7.6,3.4 \mathrm{~Hz}, 1 \mathrm{H}), 4.38(\mathrm{~d}, \mathrm{~J}=11.5 \mathrm{~Hz}, 1 \mathrm{H}), 4.45(\mathrm{~d}, \mathrm{~J}=11.5 \mathrm{~Hz}, 1 \mathrm{H}), 6.88(\mathrm{~d}, \mathrm{~J}=8.5$ $\mathrm{Hz}, 2 \mathrm{H}), 7.25(\mathrm{~d}, \mathrm{~J}=8.5 \mathrm{~Hz}, 2 \mathrm{H}), 9.69(\mathrm{~s}, 1 \mathrm{H}) ;{ }^{13} \mathrm{C} \mathrm{NMR}\left(75 \mathrm{MHz}, \mathrm{CDCl}_{3}\right): \delta 0.5,7.5,14.6,37.6,49.8,55.2$, 71.6, 72.7, 72.8, 113.7, 129.2, 130.6, 159.1, 205.0; MS (ESI): $\mathrm{m} / \mathrm{z} 361\left(\mathrm{M}^{+}+\mathrm{Na}\right)$; HRMS (ESI) $\mathrm{m} / \mathrm{z}$ calcd for $\mathrm{C}_{18} \mathrm{H}_{30} \mathrm{O}_{4} \mathrm{NaSi}\left(\mathrm{M}^{+}+\mathrm{Na}\right): 361.1811$; found: 361.1823

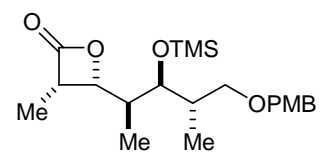

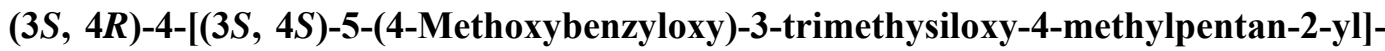
3-methyloxetan-2-one (10): To a solution of $O$-trimethylsilyquinidine (3a) (20 mg, 0.05 $\mathrm{mmol})$ and $\mathrm{LiI}(134 \mathrm{mg}, 1.0 \mathrm{mmol})$ in $0.13 \mathrm{~mL}$ of $\mathrm{Et}_{2} \mathrm{O}$ and $1.0 \mathrm{~mL} \mathrm{CH}_{2} \mathrm{Cl}_{2}$ was added $\mathrm{N}$, $N$-diisopropylethylamine $(0.22 \mathrm{~mL}, 1.25 \mathrm{mmol})$ and the solution was cooled to $-40^{\circ} \mathrm{C}$. A solution of aldehyde $9(170 \mathrm{mg}, 0.5 \mathrm{mmol})$ in $0.3 \mathrm{~mL}$ of $\mathrm{CH}_{2} \mathrm{Cl}_{2}$ was added to the reaction whereupon a solution of propionyl chloride $(0.09 \mathrm{~mL}, 1.0 \mathrm{mmol})$ in $0.4 \mathrm{~mL} \mathrm{CH}_{2} \mathrm{Cl}_{2}$ was added via syringe pump over 90 min. Once addition was complete, the reaction was stirred at $-40{ }^{\circ} \mathrm{C}$ for $16 \mathrm{~h}$ at which time the reaction was diluted with 5 $\mathrm{mL}$ of $\mathrm{Et}_{2} \mathrm{O}$. The resulting mixture was diluted with $0.1 \mathrm{~N} \mathrm{HCl}(20 \mathrm{~mL})$ and $\mathrm{CH}_{2} \mathrm{Cl}_{2}(20 \mathrm{~mL})$ and the layers were shaken and separated. The aqueous portion was extracted with $\mathrm{CH}_{2} \mathrm{Cl}_{2}(2 \times 15 \mathrm{~mL})$ and the combined organic portions were washed with saturated $\mathrm{NaHCO}_{3}$ and brine, dried and concentrated. The crude product mixture was purified by flash chromatography $\left(\mathrm{SiO}_{2}, 12 \%\right.$ ethyl acetate in hexane) to afford $166 \mathrm{mg}(84 \%)$ of the title compound as colorless oil. ${ }^{1} \mathrm{H}$ NMR analysis of the crude product revealed the diastereomer ratio to be $97: 3$ syn, anti, syn, anti: anti, anti, syn, anti. $[\alpha]_{\mathrm{D}}+10\left(c 0.84, \mathrm{CHCl}_{3}\right)$; IR (thin film): 1827, 1613, 1513, 1463, 1249, 1048, $734 \mathrm{~cm}^{-1} ;{ }^{1} \mathrm{H}$ NMR $\left(300 \mathrm{MHz}, \mathrm{CDCl}_{3}\right): \delta 0.10(\mathrm{~s}, 9 \mathrm{H}), 0.80(\mathrm{~d}, \mathrm{~J}=6.7 \mathrm{~Hz}, 3 \mathrm{H}), 0.94(\mathrm{~d}, \mathrm{~J}=6.8 \mathrm{~Hz}, 3 \mathrm{H})$, $1.31(\mathrm{~d}, \mathrm{~J}=7.7 \mathrm{~Hz}, 3 \mathrm{H}), 1.83-1.91(\mathrm{~m}, 1 \mathrm{H}), 1.95-2.00(\mathrm{~m}, 1 \mathrm{H}), 3.31$ (dd, J=8.6, 6.9 Hz, 1H), 3.50 (dd, J=8.7, 3.5 $\mathrm{Hz}, 1 \mathrm{H}), 3.80-3.74(\mathrm{~m}, 2 \mathrm{H}), 3.80(\mathrm{~s}, 3 \mathrm{H}), 4.35$ (dd, J=11.1, $6.3 \mathrm{~Hz}, 1 \mathrm{H}), 4.38(\mathrm{~d}, \mathrm{~J}=11.5 \mathrm{~Hz}, 1 \mathrm{H}), 4.48(\mathrm{~d}, \mathrm{~J}=11.5$ $\mathrm{Hz}, 1 \mathrm{H}), 6.88(\mathrm{~d}, \mathrm{~J}=8.5 \mathrm{~Hz}, 2 \mathrm{H}), 7.26(\mathrm{~d}, \mathrm{~J}=8.5 \mathrm{~Hz}, 2 \mathrm{H}) ;{ }^{13} \mathrm{C} \mathrm{NMR}\left(75 \mathrm{MHz}, \mathrm{CDCl}_{3}\right): \delta 0.7,7.8,8.5,14.4,35.3$, 37.6, 46.6, 55.3, 72.2, 72.6, 72.8, 76.2, 113.8, 129.3, 130.8, 159.2, 172.7; MS (ESI): $\mathrm{m} / \mathrm{z} 417\left(\mathrm{M}^{+}+\mathrm{Na}\right)$; HRMS (ESI) $\mathrm{m} / \mathrm{z}$ calcd for $\mathrm{C}_{21} \mathrm{H}_{34} \mathrm{O}_{5} \mathrm{NaSi}\left(\mathrm{M}^{+}+\mathrm{Na}\right)$ : 417.2073; found: 417.2058

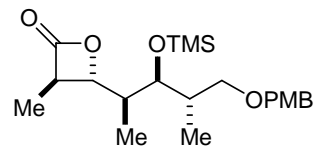

$(3 R, 4 R)-4-[(3 S, 4 S)-5-(4-M e t h o x y b e n z y l o x y)-3-t r i m e t h y s i l o x y-4-m e t h y l p e n t a n-2-y l]-$ 3-methyloxetan-2-one (11): To a solution of $O$-methylquinine (4b) $(17 \mathrm{mg}, 0.05 \mathrm{mmol})$ and LiI (167 mg, $1.25 \mathrm{mmol})$ in $0.13 \mathrm{~mL}$ of $\mathrm{Et}_{2} \mathrm{O}$ and $1.0 \mathrm{~mL} \mathrm{CH}_{2} \mathrm{Cl}_{2}$ was added $N, N$ diisopropylethylamine $(0.22 \mathrm{~mL}, 1.25 \mathrm{mmol})$ and the solution was cooled to $-40{ }^{\circ} \mathrm{C}$. A solution of aldehyde $9(170 \mathrm{mg}, 0.5 \mathrm{mmol})$ in $0.3 \mathrm{~mL}$ of $\mathrm{CH}_{2} \mathrm{Cl}_{2}$ was added to the reaction whereupon a solution of propionyl chloride $(0.9 \mathrm{~mL}, 1.0 \mathrm{mmol})$ in $0.4 \mathrm{~mL} \mathrm{CH}_{2} \mathrm{Cl}_{2}$ was added via syringe pump over 90 min. Once addition was complete, the reaction was stirred at $-40{ }^{\circ} \mathrm{C}$ for $16 \mathrm{~h}$ at which time the reaction was diluted with 5 $\mathrm{mL}$ of $\mathrm{Et}_{2} \mathrm{O}$. The resulting mixture was diluted with $0.1 \mathrm{~N} \mathrm{HCl}(20 \mathrm{~mL})$ and $\mathrm{CH}_{2} \mathrm{Cl}_{2}(20 \mathrm{~mL})$ and the layers were shaken and separated. The aqueous portion was extracted with $\mathrm{CH}_{2} \mathrm{Cl}_{2}(2 \times 15 \mathrm{~mL})$ and the combined organic portions were washed with saturated $\mathrm{NaHCO}_{3}$ and brine, dried and concentrated. The crude product mixture was purified by flash chromatography $\left(\mathrm{SiO}_{2}, 12 \%\right.$ ethyl acetate in hexane) to afford $142 \mathrm{mg}(72 \%)$ of the title compound as colorless oil. ${ }^{1} \mathrm{H}$ NMR analysis of the crude product revealed the diastereomer ratio to be 96:4 anti, anti, syn, anti:syn, anti, syn, anti. $[\alpha]_{\mathrm{D}}+28$ (c 0.73, $\left.\mathrm{CHCl}_{3}\right)$; IR (thin film): 1827, 1613, 1513, 1463, 1249, 1048, $734 \mathrm{~cm}^{-1}$; ${ }^{1} \mathrm{H}$ NMR (300 MHz, $\left.\mathrm{CDCl}_{3}\right): \delta 0.12(\mathrm{~s}, 9 \mathrm{H}), 0.84(\mathrm{~d}, \mathrm{~J}=6.9 \mathrm{~Hz}, 3 \mathrm{H}), 0.93(\mathrm{~d}, \mathrm{~J}=6.9 \mathrm{~Hz}$, $3 \mathrm{H}), 1.41(\mathrm{~d}, \mathrm{~J}=7.5 \mathrm{~Hz}, 3 \mathrm{H}), 1.79-1.90(\mathrm{~m}, 2 \mathrm{H}), 3.22$ (qd, J=7.5, 4.0 Hz, 1H), 3.33 (dd, J=8.7, 6.1 Hz, 1H), 3.49 $(\mathrm{dd}, \mathrm{J}=8.7,3.6 \mathrm{~Hz}, 1 \mathrm{H}), 3.79-3.81(\mathrm{~m}, 1 \mathrm{H}), 3.81(\mathrm{~s}, 3 \mathrm{H}), 3.97$ (dd, J=10.3, $4.0 \mathrm{~Hz}, 1 \mathrm{H}), 4.39$ (d, J=11.6 Hz, 1H), $4.49(\mathrm{~d}, \mathrm{~J}=11.6 \mathrm{~Hz}, 1 \mathrm{H}), 6.88(\mathrm{~d}, \mathrm{~J}=8.6 \mathrm{~Hz}, 2 \mathrm{H}), 7.26(\mathrm{~d}, \mathrm{~J}=8.6 \mathrm{~Hz}, 2 \mathrm{H}) ;{ }^{13} \mathrm{C} \mathrm{NMR}\left(75 \mathrm{MHz}, \mathrm{CDCl}_{3}\right): \delta$ 0.7, 7.4, 13.1, 14.4, 37.6, 40.6, 50.3, 55.3, 72.1, 72.5, 72.8, 80.3, 113.8, 129.2, 130.8, 159.2, 172.0; MS (ESI): $\mathrm{m} / \mathrm{z} 417$ $\left(\mathrm{M}^{+}+\mathrm{Na}\right)$; HRMS (ESI) $\mathrm{m} / \mathrm{z}$ calcd for $\mathrm{C}_{21} \mathrm{H}_{34} \mathrm{O}_{5} \mathrm{NaSi}\left(\mathrm{M}^{+}+\mathrm{Na}\right)$ : 417.2073; found: 417.2063.

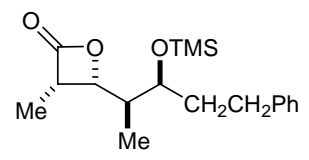

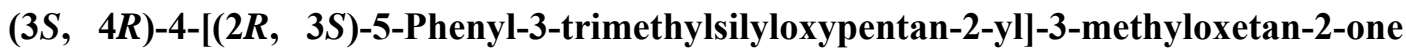
(13a): A flame-dried $25 \mathrm{~mL}$ round bottom flask was charged with $O$ trimethylsilylquinidine $(3 \mathbf{a})(45 \mathrm{mg}, 0.11 \mathrm{mmol})$ and LiI $(0.456 \mathrm{~g}, 3.41 \mathrm{mmol})$. To the 
mixture was added $\mathrm{CH}_{2} \mathrm{Cl}_{2}(3.3 \mathrm{~mL})$, DMF $(0.3 \mathrm{~mL})$ and ${ }^{i} \operatorname{Pr}_{2} \mathrm{NEt}(0.50 \mathrm{~mL}, 2.84 \mathrm{mmol})$. The resulting mixture was stirred at ambient temperature for $5 \mathrm{~min}$ then cooled to $-50{ }^{\circ} \mathrm{C}$. To the reaction mixture was added aldehyde 12a (300 mg, $1.14 \mathrm{mmol})$ whereupon a solution of propionyl chloride $(0.20 \mathrm{~mL}, 2.27 \mathrm{mmol})$ in $\mathrm{CH}_{2} \mathrm{Cl}_{2}$ $(0.76 \mathrm{~mL})$ was added via syringe pump over $2 \mathrm{~h}$. The heterogeneous reaction mixture was stirred vigorously overnight at $-50{ }^{\circ} \mathrm{C}$. The resulting mixture was partitioned between ether $(15 \mathrm{~mL})$ and $\mathrm{H}_{2} \mathrm{O}(15 \mathrm{~mL})$ and the layers were shaken and separated. The aqueous portion was extracted with ether $(2 \times)$ and the combined organic portions were washed with water $(2 \times)$ and brine. The combined organics were dried $\left(\mathrm{Na}_{2} \mathrm{SO}_{4}\right)$, filtered and concentrated and the crude residue was purified by column chromatography $\left(\mathrm{SiO}_{2}, 4 \%\right.$ ethyl acetate in hexanes) to yield $0.300 \mathrm{~g}(83 \%)$ of $\beta$-lactone 13a. ${ }^{1} \mathrm{H}$ NMR $(300 \mathrm{MHz})$ analysis of the crude product revealed 13a to be a single diastereomer (syn, anti, syn) within the limits of detection. $[\alpha]_{\mathrm{D}}=+28\left(\right.$ c 1.17, $\left.\mathrm{CHCl}_{3}\right)$; IR (thin film) 2952, 1827, 1250, 1148, 1118, 1044, $1001 \mathrm{~cm}^{-1} ;{ }^{1} \mathrm{H}$ NMR (300 MHz, $\left.\mathrm{CDCl}_{3}\right): \delta 7.33-7.18(\mathrm{~m}, 5 \mathrm{H}), 4.40(\mathrm{dd}, J=$ 11.0, $6.3 \mathrm{~Hz}, 1 \mathrm{H}), 4.01(\mathrm{ddd}, J=6.6,6.6,0.9 \mathrm{~Hz}, 1 \mathrm{H}), 3.77$ (dddd, $J=7.6,7.6,7.6,7.6 \mathrm{~Hz}, 1 \mathrm{H}), 2.67(\mathrm{ddd}, J=$ $13.5,10.8,6.0 \mathrm{~Hz}, 1 \mathrm{H}), 2.54(\mathrm{ddd}, J=13.5,10.7,6.0 \mathrm{~Hz}, 1 \mathrm{H}), 1.91-1.70(\mathrm{~m}, 3 \mathrm{H}), 1.33(\mathrm{~d}, J=7.7 \mathrm{~Hz}, 3 \mathrm{H}), 0.85$ $(\mathrm{d}, J=6.6 \mathrm{~Hz}, 3 \mathrm{H}), 0.14(\mathrm{~s}, 9 \mathrm{H}) ;{ }^{13} \mathrm{C} \mathrm{NMR}\left(75 \mathrm{MHz}, \mathrm{CDCl}_{3}\right): \delta 172.4,141.7,128.3(2 \mathrm{C}), 128.1(2 \mathrm{C}), 125.7$, 75.9, 70.1, 46.3, 37.2, 37.0, 32.5, 8.3, 7.4, 0.3 (3C); EI-MS m/z $320\left(\mathrm{M}^{+}\right), 305,249,230,207$; HRMS m/z calcd for $\mathrm{C}_{18} \mathrm{H}_{28} \mathrm{O}_{3} \mathrm{Si}: 320.1808$, found: 320.1804 .

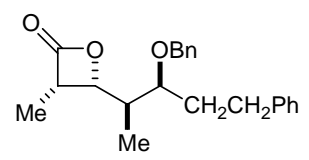

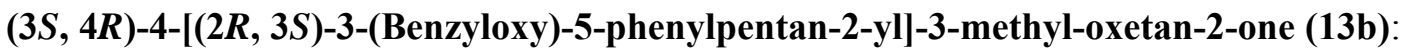
A flame-dried $25 \mathrm{~mL}$ round bottom flask was charged with $O$-trimethylsilylquinidine (3a) $(41 \mathrm{mg}, 0.10 \mathrm{mmol})$ and $\mathrm{LiClO}_{4}(0.320 \mathrm{~g}, 3.00 \mathrm{mmol})$. To the mixture was added $\mathrm{CH}_{2} \mathrm{Cl}_{2}$ $(2.8 \mathrm{~mL})$, THF $(0.3 \mathrm{~mL})$ and ${ }^{i} \operatorname{Pr}_{2} \mathrm{NEt}(0.44 \mathrm{~mL}, 2.5 \mathrm{mmol})$. The resulting mixture was stirred at ambient temperature for $5 \mathrm{~min}$ then cooled to $-40{ }^{\circ} \mathrm{C}$. To the reaction mixture was added aldehyde $12 \mathbf{b}(282 \mathrm{mg}, 1.00 \mathrm{mmol})$ whereupon a solution of propionyl chloride $(0.176 \mathrm{~mL}, 2.00 \mathrm{mmol})$ in $\mathrm{CH}_{2} \mathrm{Cl}_{2}(0.70$ $\mathrm{mL}$ ) was added via syringe pump over $4 \mathrm{~h}$. The heterogeneous reaction mixture was stirred vigorously overnight at $-40{ }^{\circ} \mathrm{C}$. The reaction was diluted ether $(20 \mathrm{~mL})$ and the resulting mixture was filtered through a silica gel plug eluting with additional ether. The filtrate was concentrated and the crude residue was purified by column chromatography $\left(\mathrm{SiO}_{2}, 5: 1\right.$ hexanes:ethyl acetate) to yield $0.257 \mathrm{~g}(76 \%)$ of $\beta$-lactone $\mathbf{1 3 b}$ as a colorless oil. IR (thin film): 2943, 1824, 1496, 1454, 1382, $1120 \mathrm{~cm}^{-1} ;{ }^{1} \mathrm{H}$ NMR $\left(300 \mathrm{MHz}, \mathrm{CDCl}_{3}\right) \delta$ 7.46-7.25 (m, 10 H), $4.74(\mathrm{~d}, \mathrm{~J}=11.5 \mathrm{~Hz}, 1 \mathrm{H}), 4.66-4.60(\mathrm{~m}, 2 \mathrm{H}), 3.90-3.75(\mathrm{~m}, 2 \mathrm{H}), 2.87$ (m, $2 \mathrm{H}), 2.23-2.03$ (m, $2 \mathrm{H}), 1.94-$ $1.82(\mathrm{~m}, 1 \mathrm{H}), 1.39(\mathrm{~d}, \mathrm{~J}=7.8 \mathrm{~Hz}, 3 \mathrm{H}), 1.01(\mathrm{~d}, \mathrm{~J}=6.8 \mathrm{~Hz}, 3 \mathrm{H}) ;{ }^{13} \mathrm{C}$ NMR $\left(75 \mathrm{MHz}, \mathrm{CDCl}_{3}\right) \delta 172.2,141.6$, 138.6, 128.2, 128.1, 128.0, 127.3, 127.2, 125.7, 77.2, 76.0, 72.7, 46.3, 36.7, 33.7, 32.2, 8.1 (note: one carbon missing; most likely hidden under $\mathrm{CDCl}_{3}$ ); HRMS m/z calcd for $\mathrm{C}_{22} \mathrm{H}_{26} \mathrm{O}_{3}: 338.1882$; found: 338.1882 .

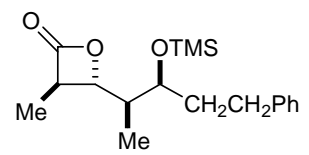

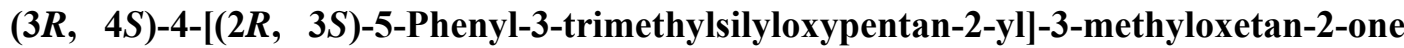
(14a): To a $-78^{\circ} \mathrm{C}$ mixture of $40 \mathrm{mg}(0.10 \mathrm{mmol}, 0.1$ equiv) of $O$-trimethylsilylquinine (4a) and $201 \mathrm{mg}\left(1.5 \mathrm{mmol}, 1.5\right.$ equiv) of LiI in $2.0 \mathrm{~mL}$ of $\mathrm{CH}_{2} \mathrm{Cl}_{2}$ and $0.25 \mathrm{~mL}$ of $\mathrm{Et}_{2} \mathrm{O}$ was added $0.44 \mathrm{~mL}$ (2.5 mmol, 2.5equiv) of $N, N$-diisopropylethylamine followed by a solution of $264 \mathrm{mg}(1.0 \mathrm{mmol})$ of aldehyde 12a in $0.75 \mathrm{~mL}$ of $\mathrm{CH}_{2} \mathrm{Cl}_{2}$. A solution of $0.175 \mathrm{~mL}(2.0 \mathrm{mmol}, 2.0$ eq) of propionyl chloride in $0.5 \mathrm{~mL}$ of $\mathrm{CH}_{2} \mathrm{Cl}_{2}$ was added to the reaction mixture over $1 \mathrm{~h}$ via syringe pump. The reaction mixture was stirred at $-78^{\circ} \mathrm{C}$ for $16 \mathrm{~h}$ and then diluted with $10 \mathrm{~mL}$ of ether. The resulting mixture was filtered through a silica gel plug eluting with $\mathrm{Et}_{2} \mathrm{O}(3 \times 20 \mathrm{~mL})$ and the filtrate was concentrated. ${ }^{1} \mathrm{H}$ NMR (300 MHz) analysis of the crude product to be a 95.5:4.5 anti, anti, syn:syn, anti, syn mixture of diastereomers. Purifying the crude residue by flash chromatography $\left(\mathrm{SiO}_{2}, 20 \%\right.$ ethyl acetate in hexane) afforded $0.259 \mathrm{~g}$ $(81 \%)$ of the title compound as colorless oil. $[\alpha]_{\mathrm{D}}+42.9$ (c 1.06, $\left.\mathrm{CHCl}_{3}\right)$; IR (thin film): 2954, 1828, 1455, $1251,1125,1067,1023 \mathrm{~cm}^{-1}$; ${ }^{1} \mathrm{H}$ NMR $\left(300 \mathrm{MHz}, \mathrm{CDCl}_{3}\right) \delta 7.35-7.20(\mathrm{~m}, 5 \mathrm{H}), 4.07(\mathrm{dd}, \mathrm{J}=9.7,4.1 \mathrm{~Hz}, 1 \mathrm{H})$, 4.00 (apparent dt, J = 6.6, $2.2 \mathrm{~Hz}, 1 \mathrm{H}), 3.28(\mathrm{dq}, \mathrm{J}=7.5,4.1 \mathrm{~Hz}, 1 \mathrm{H}), 2.73-2.50(\mathrm{~m}, 2 \mathrm{H}), 1.93-1.75(\mathrm{~m}, 3 \mathrm{H})$, $1.43(\mathrm{~d}, \mathrm{~J}=7.5 \mathrm{~Hz}, 3 \mathrm{H}), 0.92(\mathrm{~d}, \mathrm{~J}=6.9 \mathrm{~Hz}, 3 \mathrm{H}), 0.18(\mathrm{~s}, 9 \mathrm{H}) ;{ }^{13} \mathrm{C} \mathrm{NMR}\left(75 \mathrm{MHz}, \mathrm{CDCl}_{3}\right) \delta 171.8,141.7$, 128.3, 128.1, 125.8, 80.0, 70.3, 49.7, 42.1, 36.8, 32.3, 12.8, 7.3, 0.4; HRMS m/z calcd for $\mathrm{C}_{18} \mathrm{H}_{28} \mathrm{O}_{3} \mathrm{Si}: 320.1808$; found: 320.1797 .

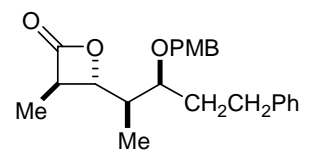

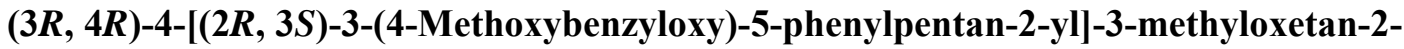
one (14c): $\mathrm{To}$ a $-78{ }^{\circ} \mathrm{C}$ mixture of $40 \mathrm{mg}$ of $O$-trimethylsilyquinine (4a) $(0.10 \mathrm{mmol}, 0.1$ 
eq)and $201 \mathrm{mg}$ ( $1.5 \mathrm{mmol}, 1.5 \mathrm{eq})$ of $\mathrm{LiI}$ in $2.0 \mathrm{~mL}$ of $\mathrm{CH}_{2} \mathrm{Cl}_{2}$ and $0.25 \mathrm{~mL}$ of $\mathrm{Et}_{2} \mathrm{O}$ was added $0.44 \mathrm{~mL}(2.5$ mmol, 2.5eq) of $N, N$-diisopropylethylamine followed by a solution of $312 \mathrm{mg}(1.0 \mathrm{mmol})$ of aldehyde $12 \mathrm{c}$ in $0.75 \mathrm{~mL}$ of $\mathrm{CH}_{2} \mathrm{Cl}_{2}$. A solution of $0.175 \mathrm{~mL}(2.0 \mathrm{mmol}, 2.0 \mathrm{eq})$ of propionyl chloride in $0.5 \mathrm{~mL}$ of $\mathrm{CH}_{2} \mathrm{Cl}_{2}$ was added to the reaction mixture over $1 \mathrm{~h}$ via syringe pump. The reaction mixture was stirred at $-78{ }^{\circ} \mathrm{C}$ for $16 \mathrm{~h}$ and then diluted with $10 \mathrm{~mL}$ of ether. The resulting mixture was filtered through a silica gel plug eluting with $\mathrm{Et}_{2} \mathrm{O}(3 \times 20 \mathrm{~mL})$ and the filtrate was concentrated. Separation of the diasteromers by HPLC [Zorbex column, flow rate $1.0 \mathrm{~mL} / \mathrm{min}, 20 \%$ EtOAc, $80 \%$ hexane, $\mathrm{T}_{\mathrm{r}} 6.4$ (trans) and 7.2 (cis) $\mathrm{min}$ ] provided the diastereomer ratio: (trans): $($ cis $)=23: 1$. Purification by flash chromatography $(20 \%$ ethyl acetate in hexane) afforded 0.300 $\mathrm{g}(81 \%)$ of the title compound as colorless oil: $[\alpha]_{\mathrm{D}}+53.1$ (c 1.68, $\left.\mathrm{CHCl}_{3}\right)$; IR (thin film): 1824, 1612, 1514, 1456, $1248 \mathrm{~cm}^{-1} ;{ }^{1} \mathrm{H}$ NMR (300 MHz, $\left.\mathrm{CDCl}_{3}\right) \delta 7.44-7.30(\mathrm{~m}, 7 \mathrm{H}), 7.03$ (app. d, J = 8.7 Hz, 2H), $4.69(\mathrm{~d}, \mathrm{~J}=$ $11.1 \mathrm{~Hz}, 1 \mathrm{H}), 4.57(\mathrm{~d}, \mathrm{~J}=11.1 \mathrm{~Hz}, 1 \mathrm{H}), 4.31(\mathrm{dd}, \mathrm{J}=9.2,4.1 \mathrm{~Hz}, 1 \mathrm{H}), 3.96(\mathrm{~s}, 3 \mathrm{H}), 3.81(\mathrm{td}, \mathrm{J}=6.6,2.6 \mathrm{~Hz}$, $1 \mathrm{H}), 3.41(\mathrm{qd}, \mathrm{J}=7.5,4.1 \mathrm{~Hz}, 1 \mathrm{H}), 2.83(\mathrm{ddd}, \mathrm{J}=13.7,10.5,5.9 \mathrm{~Hz}, 1 \mathrm{H}), 2.73(\mathrm{ddd}, \mathrm{J}=13.3,10.3,6.0 \mathrm{~Hz}, 1 \mathrm{H})$, 2.18-2.06 (m, 2H), 1.96-1.84 (m, 1H), $1.53(\mathrm{~d}, \mathrm{~J}=7.5 \mathrm{~Hz}, 3 \mathrm{H}), 1.10(\mathrm{~d}, \mathrm{~J}=7.0 \mathrm{~Hz}, 3 \mathrm{H}) ;{ }^{13} \mathrm{C} \mathrm{NMR}(75 \mathrm{MHz}$, $\left.\mathrm{CDCl}_{3}\right) \delta 171.9,159.3,141.8,130.8,129.3,128.5,128.3,126.0,113.9,80.3,77.4,72.3,55.3,49.7,41.1,33.5$, 32.3, 12.9, 8.1; HRMS m/z calcd for $\mathrm{C}_{23} \mathrm{H}_{28} \mathrm{O}_{4}$ : 368.1988; found: 368.1972 .

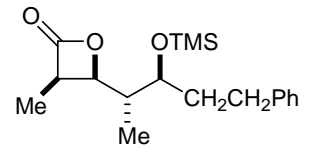

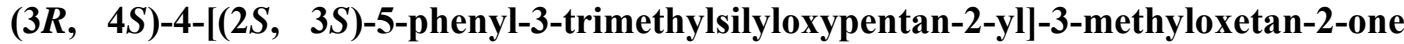
(16): A flame-dried $25 \mathrm{~mL}$ round bottom flask was charged with $O$-trimethylsilylquinine (4a) $(30 \mathrm{mg}, 0.076 \mathrm{mmol})$ and LiI $(304 \mathrm{mg}, 2.27 \mathrm{mmol})$. To the mixture was added $\mathrm{CH}_{2} \mathrm{Cl}_{2}(2.1 \mathrm{~mL})$, THF $(0.20 \mathrm{~mL})$ and ${ }^{i} \mathrm{Pr}_{2} \mathrm{NEt}(0.330 \mathrm{~mL}, 1.90 \mathrm{mmol})$. The resulting mixture was stirred at ambient temperature for $5 \mathrm{~min}$ then cooled to $-78{ }^{\circ} \mathrm{C}$. To the reaction mixture was added aldehyde 15 (200 mg, $0.76 \mathrm{mmol})$ whereupon a solution of propionyl chloride $(0.130 \mathrm{~mL}, 1.51 \mathrm{mmol})$ in $\mathrm{CH}_{2} \mathrm{Cl}_{2}$ $(0.5 \mathrm{~mL})$ was added via syringe pump over $4 \mathrm{~h}$. The heterogeneous reaction mixture was stirred vigorously overnight at $-78{ }^{\circ} \mathrm{C}$. The reaction mixture was diluted with ether $(4 \mathrm{~mL})$ and the resulting white precipitate was removed by filtration through a silica gel plug eluting with ether $(80 \mathrm{~mL})$. The filtrate was concentrated and the crude residue was purified column chromatography $\left(\mathrm{SiO}_{2}, 24: 1 /\right.$ hexanes:ethyl acetate) to yield $197 \mathrm{mg}(81 \%)$ of $\beta$-lactone 16 as a colorless solid. ${ }^{1} \mathrm{H}$ NMR $(300 \mathrm{MHz})$ analysis of the crude product revealed the product lactone to be a single diastereomer (syn, anti, anti) within the limits of detection. m.p. $=76-77^{\circ} \mathrm{C} ;[\alpha]_{\mathrm{D}}=-49(\mathrm{C}$ 1.3, $\left.\mathrm{CHCl}_{3}\right)$; IR $\left(\mathrm{CH}_{2} \mathrm{Cl}_{2}\right) 2954,1826,1250,1118,1087,1066 \mathrm{~cm}^{-1} ;{ }^{1} \mathrm{H}$ NMR $\left(300 \mathrm{MHz}, \mathrm{CDCl}_{3}\right): \delta$ 7.32-7.17 (m, 5H), 4.36 (dd, $J=11.3,6.3 \mathrm{~Hz}, 1 \mathrm{H}), 3.97-3.92(\mathrm{~m}, 1 \mathrm{H}), 3.78$ (dddd, $J=7.7,7.7,7.7,7.7 \mathrm{~Hz}, 1 \mathrm{H}), 2.80$ (ddd, $J=13.6,10.0,6.6 \mathrm{~Hz}, 1 \mathrm{H}), 2.52(\mathrm{ddd}, J=13.5,9.6,7.0 \mathrm{~Hz}, 1 \mathrm{H}), 2.10$ (ddddd, $J=10.3,6.8,6.8,6.8,3.3 \mathrm{~Hz}$, $1 \mathrm{H}), 1.80-1.70(\mathrm{~m}, 2 \mathrm{H}), 1.35(\mathrm{~d}, J=7.7 \mathrm{~Hz}, 3 \mathrm{H}), 0.89(\mathrm{~d}, J=6.8 \mathrm{~Hz}, 3 \mathrm{H}), 0.16(\mathrm{~s}, 9 \mathrm{H}) ;{ }^{13} \mathrm{C} \mathrm{NMR}(75 \mathrm{MHz}$, $\left.\mathrm{CDCl}_{3}\right): \delta 171.7,141.9,128.0$ (4C), 125.4, 75.8, 72.0, 47.1, 38.5, 33.5, 32.6, 9.5, 8.2, 0.1 (3C) ; EI-MS m/z 320 $(M)^{+}, 261,249,230,207,174$; HRMS m/z calcd for $\mathrm{C}_{18} \mathrm{H}_{28} \mathrm{O}_{3} \mathrm{Si}: 320.1808$, found 320.1825 .

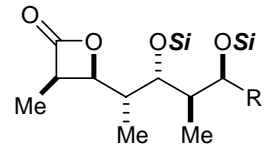

$(3 R, 4 S)-4-[(2 S, 3 R, 4 R, 5 S)-3,5-B i s(t r i m e t h y l s i l y l o x y)-4-m e t h y l-7-p h e n y l h e p t a n-2-y l]-3-$ methyloxetan-2-one (18): A flame-dried $25 \mathrm{~mL}$ round bottom flask was charged with $O$ trimethylsilylquinine (4a) $(29 \mathrm{mg}, 72.0 \mu \mathrm{mol})$ and LiI (289 mg, $2.16 \mathrm{mmol})$. To the mixture of solids was added $\mathrm{CH}_{2} \mathrm{Cl}_{2}(2.1 \mathrm{~mL}), \mathrm{Et}_{2} \mathrm{O}(0.20 \mathrm{~mL})$ and ${ }^{i} \operatorname{Pr}_{2} \mathrm{NEt}(0.31 \mathrm{~mL}, 1.80 \mathrm{mmol})$. The resulting mixture was stirred at ambient temperature for $5 \mathrm{~min}$ then cooled to $-50{ }^{\circ} \mathrm{C}$. To the reaction mixture was added aldehyde $17(284 \mathrm{mg}, 0.72 \mathrm{mmol})$ whereupon a solution of propionyl chloride $(0.130 \mathrm{~mL}, 1.44 \mathrm{mmol})$ in $\mathrm{CH}_{2} \mathrm{Cl}_{2}(0.48 \mathrm{~mL})$ was added via syringe pump over $2 \mathrm{~h}$. The heterogeneous reaction mixture was stirred vigorously overnight at $-50{ }^{\circ} \mathrm{C}$. The reaction mixture was diluted with ether and filtered through a silica gel plug eluting with ether. The filtrate was concentrated and the crude residue was purified by column chromatography $\left(\mathrm{SiO}_{2}, 24: 1 /\right.$ hexanes:ethyl acetate) to yield $258 \mathrm{mg}$ (79\%) of $\beta$-lactone 18: ${ }^{1} \mathrm{H}$ NMR $(300 \mathrm{MHz})$ analysis of the crude product revealed $\mathbf{1 8}$ to be a single diastereomer (syn, anti, syn, anti, syn) within the limits of detection. $[\alpha]_{\mathrm{D}}=-4.0\left(\right.$ c 1.0, $\left.\mathrm{CHCl}_{3}\right)$; IR (thin film) 2954, 1828, 1250, 1148, 1117, 1080, 1050, $1002 \mathrm{~cm}^{-1}$; ${ }^{1} \mathrm{H} \mathrm{NMR}\left(300 \mathrm{MHz}, \mathrm{CDCl}_{3}\right): \delta 7.32-7.18(\mathrm{~m}, 5 \mathrm{H}), 4.37(\mathrm{dd}, J=11.1,6.3 \mathrm{~Hz}, 1 \mathrm{H}), 3.95-$ $3.91(\mathrm{~m}, 2 \mathrm{H}), 3.77$ (dddd, $J=14.1,7.7,7.7,7.7 \mathrm{~Hz}, 1 \mathrm{H}), 2.59(\mathrm{dd}, J=10.1,6.4 \mathrm{~Hz}, 2 \mathrm{H}), 2.00-1.82(\mathrm{~m}, 3 \mathrm{H})$, $1.74-1.65(\mathrm{~m}, 1 \mathrm{H}), 1.34(\mathrm{~d}, J=7.8 \mathrm{~Hz}, 3 \mathrm{H}), 0.83(\mathrm{~d}, J=7.0 \mathrm{~Hz}, 3 \mathrm{H}), 0.82(\mathrm{~d}, J=6.6 \mathrm{~Hz}, 3 \mathrm{H}), 0.14(\mathrm{~s}, 18 \mathrm{H}) ;{ }^{13} \mathrm{C}$ $\operatorname{NMR}\left(75 \mathrm{MHz}, \mathrm{CDCl}_{3}\right): \oint 172.1,141.8,128.2$ (2C), 128.0 (2C), 125.6, 75.9, 72.3, 71.7, 46.3, 41.2, 37.3, 35.2, 
32.0, 9.6, 8.2, 7.9, $1.2(3 \mathrm{C}), 0.6(3 \mathrm{C})$; ESI-MS m/z $473(\mathrm{M}+\mathrm{Na})^{+}$; HRMS $\mathrm{m} / \mathrm{z}$ calcd for $\mathrm{C}_{24} \mathrm{H}_{42} \mathrm{O}_{4} \mathrm{NaSi}_{2}(\mathrm{M}+\mathrm{Na})^{+}$: 473.2519 , found 473.2532 .

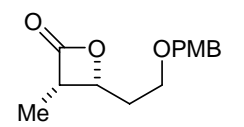

(3S, 4R)-4-[2-(4-Methoxybenzyloxy)ethyl]-3-methyloxetan-2-one (20): To a solution of $O$ trimethylsilylquinidine (3a) $(0.926 \mathrm{~g}, 2.32 \mathrm{mmol})$ and $\mathrm{LiClO}_{4}(0.737 \mathrm{~g}, 6.95 \mathrm{mmol})$ in $23 \mathrm{~mL}$ of diethyl ether was added $46 \mathrm{~mL}$ of $\mathrm{CH}_{2} \mathrm{Cl}_{2}$ and the reaction mixture was cooled to $-78{ }^{\circ} \mathrm{C}$. To the resulting mixture was added $N, N$-diisopropylethylamine $(10.0 \mathrm{~mL}, 57.9 \mathrm{mmol})$ followed by $3-(4-$ methoxybenzyloxy)propionaldehyde $(4.49 \mathrm{~g}, 23.2 \mathrm{mmol})$. A solution of propionyl chloride $(4.0 \mathrm{~mL}, 46.4$ mmol) in $6.0 \mathrm{~mL} \mathrm{CH}_{2} \mathrm{Cl}_{2}$ was then added over $1 \mathrm{~h}$ by syringe pump. The reaction mixture was stirred for $18 \mathrm{~h}$ then quenched at $-78{ }^{\circ} \mathrm{C}$ by adding $100 \mathrm{~mL}$ of $\mathrm{Et}_{2} \mathrm{O}$ and $100 \mathrm{~mL}$ saturated aqueous $\mathrm{NH}_{4} \mathrm{Cl}$. The layers were shaken and separated and the aqueous portion was extracted with $\mathrm{Et}_{2} \mathrm{O}(3 \times 100 \mathrm{~mL})$. The combined organic portions were washed with brine, dried $\left(\mathrm{Na}_{2} \mathrm{SO}_{4}\right)$ and concentrated. The crude product mixture was purified by flash chromatography $\left(\mathrm{SiO}_{2}, 15 \%\right.$ ethyl acetate in hexane) to give $4.06 \mathrm{~g}(70 \%)$ of the title compound as a colorless oil (syn: anti $=89: 11$ ). Separating the enantiomers by chiral HPLC [Daicel Chiracel ${ }^{\mathrm{TM}}$ OD-H column, flow rate $1.0 \mathrm{~mL} / \mathrm{min}, 10 \%{ }^{i} \mathrm{PrOH}, 90 \%$ hexane, $\left.\mathrm{T}_{\mathrm{r}}: 13.3 \mathrm{~min}(3 R, 4 S), 18.7 \mathrm{~min}(3 \mathrm{~S}, 4 R)\right]$ provided only the $(3 S$, $4 R$ ) enantiomer (99\% ee). $[\alpha]_{\mathrm{D}}+38.8$ (c 1.81, $\left.\mathrm{CHCl}_{3}\right)$; IR (thin film): $1817,1613,1514,1248 \mathrm{~cm}^{-1} ;{ }^{1} \mathrm{H}$ NMR $\left(300 \mathrm{MHz}, \mathrm{CDCl}_{3}\right) \delta 7.26(\mathrm{~d}, \mathrm{~J}=8.6 \mathrm{~Hz}, 2 \mathrm{H}), 6.89(\mathrm{~d}, \mathrm{~J}=8.7 \mathrm{~Hz}, 2 \mathrm{H}), 4.79$ (ddd, J = 8.2, 6.5, 5.2 Hz, 1H), 4.46 $(\mathrm{s}, 2 \mathrm{H}), 3.82(\mathrm{~s}, 3 \mathrm{H}), 3.77(\mathrm{dq}, \mathrm{J}=7.7,6.5 \mathrm{~Hz}, 1 \mathrm{H}), 3.67-3.56(\mathrm{~m}, 2 \mathrm{H}), 2.05-1.96(\mathrm{~m}, 2 \mathrm{H}), 1.28(\mathrm{~d}, \mathrm{~J}=7.8 \mathrm{~Hz}, 3$ $\mathrm{H}) ;{ }^{13} \mathrm{C}$ NMR $\left(75 \mathrm{MHz}, \mathrm{CDCl}_{3}\right) \delta 172.1,158.8,129.7,128.8,113.3,72.5,72.3,65.2,54.7,46.8,30.0,7.7$; HRMS $m / z$ calcd for $\mathrm{C}_{14} \mathrm{H}_{18} \mathrm{O}_{4}: 250.1205$; found: 250.1203 .

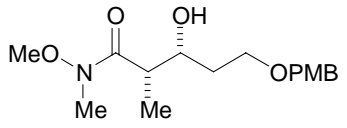

(2S, 3R)- $N$-Methoxy- $N$-methyl-3-hydroxy-5-(4-methoxybenzyloxy)-2-methylpentamide $\left(\mathbf{2 0}^{\prime}\right)$ : To a suspension of $N$, $O$-dimethylhydroxylamine hydrochloride $(2.26 \mathrm{~g}$, $23.2 \mathrm{mmol})$ in $40 \mathrm{~mL} \mathrm{CH} \mathrm{Cl}_{2}$ at $0{ }^{\circ} \mathrm{C}$ was added dimethylaluminium chloride $(1.0 \mathrm{M}$ solution in hexanes, $23.2 \mathrm{~mL}, 23.2 \mathrm{mmol}$ ) dropwise. The resulting mixture was warmed to ambient temperature and stirred for $2 \mathrm{~h}$, then cooled to $-25{ }^{\circ} \mathrm{C}$ and a solution of $\mathbf{2 0}(2.90 \mathrm{~g}, 11.6 \mathrm{mmol})$ in $30 \mathrm{~mL} \mathrm{CH}_{2} \mathrm{Cl}_{2}$ was added dropwise. The reaction mixture was stirred $2 \mathrm{~h}$ then was quenched at $-25{ }^{\circ} \mathrm{C}$ by adding $50 \mathrm{~mL}$ of aqueous Rochelle's salt solution. The resulting mixture was extracted with $\mathrm{CH}_{2} \mathrm{Cl}_{2}(3 \times 100 \mathrm{~mL})$. The combined organic extracts were washed with brine, dried $\left(\mathrm{Na}_{2} \mathrm{SO}_{4}\right)$ and concentrated. The crude product was used without further purification. $[\alpha]_{\mathrm{D}}+10.1$ (c 1.92, $\mathrm{CHCl}_{3}$ ); IR (thin film): 3459 (br), 1655, 1248 $\mathrm{cm}^{-1} ;{ }^{1} \mathrm{H} \mathrm{NMR}\left(300 \mathrm{MHz}, \mathrm{CDCl}_{3}\right)$ $\delta 7.26(\mathrm{~d}, \mathrm{~J}=8.6 \mathrm{~Hz}, 2 \mathrm{H}), 6.87(\mathrm{~d}, \mathrm{~J}=8.7 \mathrm{~Hz}, 2 \mathrm{H}), 4.45(\mathrm{~s}, 2 \mathrm{H}), 4.05(\mathrm{dt}, \mathrm{J}=9.1,3.7 \mathrm{~Hz}, 1 \mathrm{H}), 3.93(\mathrm{~s}, 1 \mathrm{H}), 3.81$ $(\mathrm{s}, 3 \mathrm{H}), 3.67(\mathrm{~s}, 3 \mathrm{H}), 3.67-3.63(\mathrm{~m}, 2 \mathrm{H}), 3.19(\mathrm{~s}, 3 \mathrm{H}), 2.92(\mathrm{br}, 1 \mathrm{H}), 1.89-1.78(\mathrm{~m}, 1 \mathrm{H}), 1.73-1.63(\mathrm{~m}, 1 \mathrm{H}), 1.20$ $(\mathrm{d}, \mathrm{J}=7.0 \mathrm{~Hz}, 3 \mathrm{H}) ;{ }^{13} \mathrm{C} \mathrm{NMR}\left(75 \mathrm{MHz}, \mathrm{CDCl}_{3}\right) \delta 177.1,158.8,130.0,128.8,113.3,72.4,70.1,67.6,61.1,54.8$, 39.3, 33.7, 31.5, 11.1; HRMS m/z calcd for $\mathrm{C}_{16} \mathrm{H}_{25} \mathrm{NO}_{5}$ : 311.1733; found: 311.1733 .

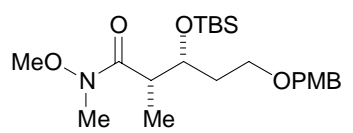

(2S, 3R)- $N$-Methoxy- $N$-methyl-3-(tert-butyldimethylsilanyloxy)-5-(4-methoxybenzyloxy)-2-methylpentamide (20"): To a solution of $\mathbf{2 0}^{\prime}$ (11.6 mmol crude) in 35 $\mathrm{mL}$ of DMF at ambient temperature was added imidazole $(1.71 \mathrm{~g}, 25.1 \mathrm{mmol})$ followed by TBSCl $(3.15 \mathrm{~g}, 20.9 \mathrm{mmol})$ and DMAP $(142 \mathrm{mg}, 1.16 \mathrm{mmol})$. The reaction mixture was stirred for $20 \mathrm{~h}$ then diluted with $100 \mathrm{~mL}$ saturated aqueous $\mathrm{NH}_{4} \mathrm{Cl}$. The mixture was extracted with $\mathrm{CH}_{2} \mathrm{Cl}_{2}(3 \times 150 \mathrm{~mL})$. The combined organic extracts were washed with brine, dried $\left(\mathrm{Na}_{2} \mathrm{SO}_{4}\right)$ and concentrated. The crude product mixture was purified by flash chromatography $\left(\mathrm{SiO}_{2}, 10 \%\right.$ ethyl acetate in hexane) to give $4.80 \mathrm{~g}(97 \%$ over 2 steps) of the title compound as a colorless oil. $[\alpha]_{\mathrm{D}}+0.30\left(c \mathrm{1.76}, \mathrm{CHCl}_{3}\right)$; IR (thin film): 1661, 1514, 1250, 837, $776 \mathrm{~cm}^{-1}$; ${ }^{1} \mathrm{H}$ NMR $\left(300 \mathrm{MHz}, \mathrm{CDCl}_{3}\right) \delta 7.25(\mathrm{~d}, \mathrm{~J}=8.8 \mathrm{~Hz}, 2 \mathrm{H}), 6.87(\mathrm{~d}, \mathrm{~J}=8.7 \mathrm{~Hz}, 2 \mathrm{H}), 4.41(\mathrm{~s}, 2 \mathrm{H})$, $4.03(\mathrm{dt}, \mathrm{J}=7.9,4.9 \mathrm{~Hz}, 1 \mathrm{H}), 3.81(\mathrm{~s}, 3 \mathrm{H}), 3.61(\mathrm{~s}, 3 \mathrm{H}), 3.58-3.49(\mathrm{~m}, 2 \mathrm{H}), 3.15(\mathrm{~s}, 3 \mathrm{H}), 3.04-2.96(\mathrm{~m}, 1 \mathrm{H})$, 1.86-1.80 (m, 2H), $1.14(\mathrm{~d}, \mathrm{~J}=6.9,3 \mathrm{H}), 0.89(\mathrm{~s}, 9 \mathrm{H}), 0.062(\mathrm{~s}, 3 \mathrm{H}), 0.050(\mathrm{~s}, 3 \mathrm{H}) ;{ }^{13} \mathrm{C}$ NMR $\left(75 \mathrm{MHz}, \mathrm{CDCl}_{3}\right)$ $\delta 176.1,159.1,130.6,129.2,113.6,72.4,71.3,66.2,61.1,55.2,41.2,35.4,32.1,25.9,18.0,14.3,-4.4,-4.5$; HRMS $\mathrm{m} / \mathrm{z}$ calcd for $\left(\mathrm{M}^{+}+\mathrm{Na}\right) \mathrm{C}_{22} \mathrm{H}_{39} \mathrm{NO}_{5} \mathrm{NaSi}$ : 448.2495; found: 448.2452.

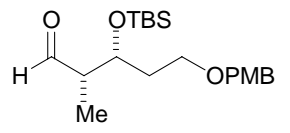

(2S, 3R)-3-(tert-Butyldimethylsilanyloxy)-5-(4-methoxybenzyloxy)-2-methylpentanal (21): To a solution of 20" $(4.45 \mathrm{~g}, 10.45 \mathrm{mmol})$ in $50 \mathrm{~mL}$ of $\mathrm{THF}$ at $-50{ }^{\circ} \mathrm{C}$ was added ${ }^{i} \mathrm{Bu}_{2} \mathrm{AlH}(1.0 \mathrm{M}$ in hexanes, $13.0 \mathrm{~mL}, 13.0 \mathrm{mmol})$ over $30 \mathrm{~min}$ by syringe pump. The reaction mixture was stirred for $30 \mathrm{~min}$ then was quenched by adding $100 \mathrm{~mL}$ aqueous Rochelle's salt solution. 
The mixture was extracted with $\mathrm{Et}_{2} \mathrm{O}(3 \times 100 \mathrm{~mL})$. The combined organic extracts were washed with brine, dried $\left(\mathrm{Na}_{2} \mathrm{SO}_{4}\right)$ and concentrated. The crude product mixture was purified by flash chromatography $\left(\mathrm{SiO}_{2}, 11 \%\right.$ ethyl acetate in hexane) to give $3.68 \mathrm{~g}(96 \%)$ of the title compound as a colorless oil. $[\alpha]_{\mathrm{D}}+47.7$ (c 1.46, $\mathrm{CHCl}_{3}$ ); IR (thin film): 1726, 1613, 1514, 1250, 837, $776 \mathrm{~cm}^{-1} ;{ }^{1} \mathrm{H}$ NMR $\left(300 \mathrm{MHz}, \mathrm{CDCl}_{3}\right) \delta 9.78(\mathrm{~s}, 1 \mathrm{H}), 7.26$ $(\mathrm{d}, \mathrm{J}=8.7 \mathrm{~Hz}, 2 \mathrm{H}), 6.89(\mathrm{~d}, \mathrm{~J}=8.6 \mathrm{~Hz}, 2 \mathrm{H}), 4.45(\mathrm{~d}, \mathrm{~J}=11.5 \mathrm{~Hz}, 1 \mathrm{H}), 4.39(\mathrm{~d}, \mathrm{~J}=11.5 \mathrm{~Hz}, 1 \mathrm{H}), 4.31$ (ddd, J = 7.2, 5.7, 3.7 Hz, 1H), $3.82(\mathrm{~s}, 3 \mathrm{H}), 3.49(\mathrm{t}, \mathrm{J}=5.7 \mathrm{~Hz}, 2 \mathrm{H}), 2.53-2.44(\mathrm{~m}, 1 \mathrm{H}), 1.82-1.77(\mathrm{~m}, 2 \mathrm{H}), 1.06(\mathrm{~d}, \mathrm{~J}=$ $7.0 \mathrm{~Hz}, 3 \mathrm{H}), 0.87(\mathrm{~s}, 9 \mathrm{H}), 0.072(\mathrm{~s}, 3 \mathrm{H}), 0.047(\mathrm{~s}, 3 \mathrm{H}) ;{ }^{13} \mathrm{C} \mathrm{NMR}\left(75 \mathrm{MHz}, \mathrm{CDCl}_{3}\right) \delta 204.3,159.0,130.1,129.0$, $113.5,72.4,69.0,65.9,54.8,51.3,34.4,25.5,17.7,7.5,-4.7,-4.9$; HRMS m/z calcd for $\left(\mathrm{M}^{+}+\mathrm{Na}\right)$ $\mathrm{C}_{20} \mathrm{H}_{34} \mathrm{O}_{4} \mathrm{SiNa}: 389.2124$; found: 389.2114 .

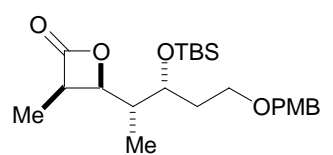

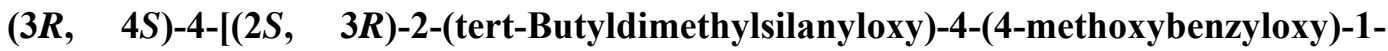
methylbutyl]-3-methyloxetan-2-one (22): To a suspension of $O$-trimethylsilylquinine (4a) $(397 \mathrm{mg}, 0.99 \mathrm{mmol})$ and LiI $(6.64 \mathrm{~g}, 49.59 \mathrm{mmol})$ in $4.5 \mathrm{~mL}$ of diethyl ether was added $40.0 \mathrm{~mL}$ of $\mathrm{CH}_{2} \mathrm{Cl}_{2}$ and the reaction mixture was cooled to $-78{ }^{\circ} \mathrm{C}$. To the resulting mixture was added $N, N$-diisopropylethylamine $(4.3 \mathrm{~mL}, 24.9 \mathrm{mmol})$ followed by a solution of 21 (3.43 g, $9.92 \mathrm{mmol})$ in $8.0 \mathrm{~mL} \mathrm{CH}_{2} \mathrm{Cl}_{2}$. A solution of propionyl chloride $(1.73 \mathrm{~mL}, 20.0 \mathrm{mmol})$ in $6.0 \mathrm{~mL}$ $\mathrm{CH}_{2} \mathrm{Cl}_{2}$ was then added over $3 \mathrm{~h}$ by syringe pump. After the addition of propionyl chloride was complete, the reaction was quenched at the $-78{ }^{\circ} \mathrm{C}$ by adding $100 \mathrm{~mL}$ of $\mathrm{Et}_{2} \mathrm{O}$ and $100 \mathrm{~mL}$ saturated aqueous $\mathrm{NH}_{4} \mathrm{Cl}$. The resulting mixture was extracted with $\mathrm{Et}_{2} \mathrm{O}(3 \times 100 \mathrm{~mL})$ and the combined organic extracts were washed with brine, dried $\left(\mathrm{Na}_{2} \mathrm{SO}_{4}\right)$ and concentrated. The crude product mixture was purified by flash chromatography $\left(\mathrm{SiO}_{2}, 9 \%\right.$ ethyl acetate in hexane) to give $3.60 \mathrm{~g}(91 \%)$ of the title compound as a single diastereomer. $[\alpha]_{\mathrm{D}}$ -20.3 (c 2.28, $\mathrm{CHCl}_{3}$ ); IR (thin film): $1824,1613,1250 \mathrm{~cm}^{-1} ;{ }^{1} \mathrm{H}$ NMR $\left(300 \mathrm{MHz}, \mathrm{CDCl}_{3}\right) \delta 7.25$ (d, J $=8.7 \mathrm{~Hz}$, $2 \mathrm{H}), 6.88(\mathrm{~d}, \mathrm{~J}=8.6 \mathrm{~Hz}, 2 \mathrm{H}), 4.47(\mathrm{~d}, \mathrm{~J}=11.6 \mathrm{~Hz}, 1 \mathrm{H}), 4.41(\mathrm{dd}, \mathrm{J}=11.1,6.3 \mathrm{~Hz}, 1 \mathrm{H}), 4.36(\mathrm{~d}, \mathrm{~J}=11.4 \mathrm{~Hz}$, $1 \mathrm{H}), 4.10(\mathrm{t}, \mathrm{J}=6.2 \mathrm{~Hz}, 1 \mathrm{H}), 3.82(\mathrm{~s}, 3 \mathrm{H}), 3.75(\mathrm{~m}, 1 \mathrm{H}), 3.43(\mathrm{t}, \mathrm{J}=6.4 \mathrm{~Hz}, 2 \mathrm{H}), 1.88-1.78(\mathrm{~m}, 3 \mathrm{H}), 1.26(\mathrm{~d}, \mathrm{~J}=$ $7.7 \mathrm{~Hz}, 3 \mathrm{H}), 0.89(\mathrm{~s}, 9 \mathrm{H}), 0.81(\mathrm{~d}, \mathrm{~J}=6.7 \mathrm{~Hz}, 3 \mathrm{H}), 0.065(\mathrm{~s}, 6 \mathrm{H}) ;{ }^{13} \mathrm{C} \mathrm{NMR}\left(75 \mathrm{MHz}, \mathrm{CDCl}_{3}\right) \delta 172.6,159.0$, $130.2,129.1,113.7,75.9,72.5,67.5,66.4,55.1,46.3,37.0,35.0,25.8,18.0,8.2,7.3,-4.5,-5.0$; HRMS $\mathrm{m} / \mathrm{z}$ calcd for $\left(\mathrm{M}^{+}-{ }^{t} \mathrm{Bu}\right) \mathrm{C}_{19} \mathrm{H}_{29} \mathrm{O}_{5} \mathrm{Si}: 365.1784$; found: 365.1786 .

OPMB

$(2 S, 3 R, 4 S, 5 R)-5-(t e r t-B u t y l d i m e t h y l s i l a n y l o x y)-7-(4-m e t h o x y b e n z y l o x y)-2,4-$ dimethylheptane-1,3-diol (22'): To a solution of $22(3.60 \mathrm{~g}, 8.52 \mathrm{mmol})$ in $60 \mathrm{~mL}$ of THF at $-50{ }^{\circ} \mathrm{C}$ was added ${ }^{i} \mathrm{Bu}_{2} \mathrm{AlH}(1.0 \mathrm{M}$ in hexanes, $25.6 \mathrm{~mL}, 25.6 \mathrm{mmol})$ over 30 min by syringe pump. The reaction mixture was stirred for 10 min then $50 \mathrm{~mL}$ of $\mathrm{Et}_{2} \mathrm{O}$ and $50 \mathrm{~mL}$ aqueous Rochelle's salt solution were added. The reaction mixture was extracted with $\mathrm{Et}_{2} \mathrm{O}(3 \times 100 \mathrm{~mL})$ and the combined organic extracts were washed with brine, dried $\left(\mathrm{Na}_{2} \mathrm{SO}_{4}\right)$ and concentrated. The crude product was used without further purification. $[\alpha]_{\mathrm{D}}+33.0$ (c 1.99, $\mathrm{CHCl}_{3}$ ); IR (thin film): 3465 (br), 1514, 1250, 837, 778 $\mathrm{cm}^{-1} ;{ }^{1} \mathrm{H}$ NMR $\left(300 \mathrm{MHz}, \mathrm{CDCl}_{3}\right) \delta 7.26,(\mathrm{~d}, \mathrm{~J}=8.6 \mathrm{~Hz}, 2 \mathrm{H}), 6.89(\mathrm{~d}, \mathrm{~J}=8.7 \mathrm{~Hz}, 2 \mathrm{H}), 4.46(\mathrm{~d}, \mathrm{~J}=11.5 \mathrm{~Hz}$, $1 \mathrm{H}), 4.40(\mathrm{~d}, \mathrm{~J}=11.4 \mathrm{~Hz}, 1 \mathrm{H}), 4.00-3.96(\mathrm{~m}, 1 \mathrm{H}), 3.94(\mathrm{dd}, \mathrm{J}=10.3,2.0 \mathrm{~Hz}, 1 \mathrm{H}), 3.82(\mathrm{~s}, 3 \mathrm{H}), 3.80(\mathrm{dd}, \mathrm{J}=$ $10.5,3.6 \mathrm{~Hz}, 1 \mathrm{H}), 3.68(\mathrm{dd}, \mathrm{J}=10.6,5.4 \mathrm{~Hz}, 1 \mathrm{H}), 3.60-3.48(\mathrm{~m}, 2 \mathrm{H}), 1.94-1.83(\mathrm{~m}, 3 \mathrm{H}), 1.66-1.63(\mathrm{~m}, 1 \mathrm{H})$, $0.96(\mathrm{~d}, \mathrm{~J}=7.0 \mathrm{~Hz}, 3 \mathrm{H}), 0.90(\mathrm{~s}, 9 \mathrm{H}), 0.75(\mathrm{~d}, \mathrm{~J}=7.1 \mathrm{~Hz}, 3 \mathrm{H}), 0.133(\mathrm{~s}, 3 \mathrm{H}), 0.074(\mathrm{~s}, 3 \mathrm{H}) ;{ }^{13} \mathrm{C} \mathrm{NMR}(75 \mathrm{MHz}$, $\left.\mathrm{CDCl}_{3}\right) \delta 159.1,130.4,129.2,113.7,76.0,74.9,72.5,67.8,66.6,55.2,39.7,36.2,31.1,25.6,17.8,13.4,8.3$, $-4.5,-5.1$.

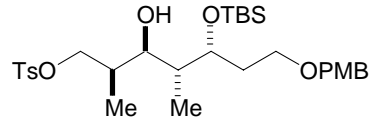

$(2 S, 3 R, 4 S, 5 R)-5$-(tert-Butyldimethylsilanyloxy)-3-hydroxy-7-(4-methoxybenzyloxy)-2, 4-dimethylheptyl 4-methylbenzenesulfonate (22"): To a solution of 22' (1.81 g, $4.24 \mathrm{mmol}$ ) in $40 \mathrm{~mL}$ of $\mathrm{CH}_{2} \mathrm{Cl}_{2}$ at ambient temperature was added pyridine (840 mg, $10.6 \mathrm{mmol})$ followed by DMAP $(104 \mathrm{mg}, 0.85 \mathrm{mmol})$ and $\mathrm{TsCl}(1.21 \mathrm{~g}, 6.37 \mathrm{mmol})$. The reaction

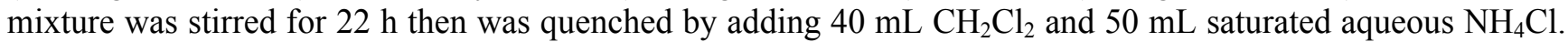
The mixture was extracted with $\mathrm{CH}_{2} \mathrm{Cl}_{2}(3 \times 80 \mathrm{~mL})$ and the combined organic extracts were washed with brine, dried $\left(\mathrm{Na}_{2} \mathrm{SO}_{4}\right)$ and concentrated. The crude product mixture was purified by flash chromatography $\left(\mathrm{SiO}_{2}, 7 \%\right.$ ethyl acetate in hexane) to give $2.05 \mathrm{~g}(83 \%$ over 2 steps $)$ of the title compound as a colorless oil. $[\alpha]_{\mathrm{D}}+18.6(\mathrm{c}$ 1.86, $\mathrm{CHCl}_{3}$ ); IR (thin film): 3469 (br), 1613, 1514, 1361, 1249, 964, $838 \mathrm{~cm}^{-1} ;{ }^{1} \mathrm{H}$ NMR $\left(300 \mathrm{MHz}, \mathrm{CDCl}_{3}\right)$ $\delta 7.80(\mathrm{~d}, \mathrm{~J}=8.3 \mathrm{~Hz}, 2 \mathrm{H}), 7.33(\mathrm{~d}, \mathrm{~J}=8.0 \mathrm{~Hz}, 2 \mathrm{H}), 7.26(\mathrm{~d}, \mathrm{~J}=8.7 \mathrm{~Hz}, 2 \mathrm{H}), 6.89(\mathrm{~d}, \mathrm{~J}=8.7 \mathrm{~Hz}, 2 \mathrm{H}), 4.45(\mathrm{~d}, \mathrm{~J}$ $=11.5 \mathrm{~Hz}, 1 \mathrm{H}), 4.39(\mathrm{~d}, \mathrm{~J}=11.5 \mathrm{~Hz}, 1 \mathrm{H}), 4.12(\mathrm{~d}, \mathrm{~J}=7.3 \mathrm{~Hz}, 1 \mathrm{H}), 4.09(\mathrm{~d}, \mathrm{~J}=7.2 \mathrm{~Hz}, 1 \mathrm{H}), 3.98-3.91(\mathrm{~m}, 2 \mathrm{H})$, 
$3.82(\mathrm{~s}, 3 \mathrm{H}), 3.70(\mathrm{dd}, \mathrm{J}=10.2,1.7 \mathrm{~Hz}, 1 \mathrm{H}), 3.56-3.47(\mathrm{~m}, 2 \mathrm{H}), 2.44(\mathrm{~s}, 3 \mathrm{H}), 1.92-1.70(\mathrm{~m}, 4 \mathrm{H}), 0.87(\mathrm{~s}, 9 \mathrm{H})$, $0.84(\mathrm{~d}, \mathrm{~J}=6.8 \mathrm{~Hz}, 3 \mathrm{H}), 0.69(\mathrm{~d}, \mathrm{~J}=7.1 \mathrm{~Hz}, 3 \mathrm{H}), 0.094(\mathrm{~s}, 3 \mathrm{H}), 0.057(\mathrm{~s}, 3 \mathrm{H}) ;{ }^{13} \mathrm{C} \mathrm{NMR}\left(75 \mathrm{MHz}, \mathrm{CDCl}_{3}\right)$ $\delta 159.0,144.3,133.2,130.3,129.6,129.1,127.7,113.6,74.6,73.5,72.4,71.7,66.4,55.1,39.3,35.2,31.0,25.6$, 21.4, 17.7, 13.0, 8.1, -4.6, -5.2; HRMS $m / z$ calcd for $\left(\mathrm{M}^{+}+\mathrm{Na}\right) \mathrm{C}_{30} \mathrm{H}_{48} \mathrm{O}_{7} \mathrm{SSiNa}$ : 603.2788; found: 603.2778 .

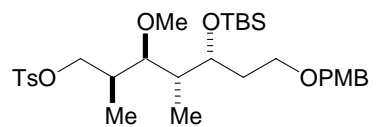

$(3.20 \mathrm{~g}, 21.6 \mathrm{mmol})$ followed by $\mathrm{Me}_{3} \mathrm{OBF}_{4}(4.626 \mathrm{~g}, 21.6 \mathrm{mmol})$. The reaction flask was covered with aluminum foil and the reaction stirred for $8 \mathrm{~h}$. The reaction was quenched by adding $100 \mathrm{~mL}$ of $\mathrm{CH}_{2} \mathrm{Cl}_{2}$ and 150 $\mathrm{mL}$ of saturated aqueous $\mathrm{NH}_{4} \mathrm{Cl}$. The mixture was extracted with $\mathrm{CH}_{2} \mathrm{Cl}_{2}(3 \times 100 \mathrm{~mL})$ and the combined organic extracts were washed with brine, dried $\left(\mathrm{Na}_{2} \mathrm{SO}_{4}\right)$ and concentrated. The crude product mixture was purified by flash chromatography $\left(\mathrm{SiO}_{2}, 8 \%\right.$ ethyl acetate in hexane) to give $1.73 \mathrm{~g}(81 \%)$ of the title compound as a colorless oil. $[\alpha]_{\mathrm{D}}-0.67$ (c 1.79, $\mathrm{CHCl}_{3}$ ); IR (thin film): 1514, 1365, 1249, 1189, 1178, $835 \mathrm{~cm}^{-1} ;{ }^{1} \mathrm{H}$ NMR $\left(300 \mathrm{MHz}, \mathrm{CDCl}_{3}\right) \delta 7.81(\mathrm{~d}, \mathrm{~J}=8.3 \mathrm{~Hz}, 2 \mathrm{H}), 7.35(\mathrm{~d}, \mathrm{~J}=8.0 \mathrm{~Hz}, 2 \mathrm{H}), 7.25(\mathrm{~d}, \mathrm{~J}=8.7 \mathrm{~Hz}, 2 \mathrm{H}), 6.87(\mathrm{~d}, \mathrm{~J}=8.7$ $\mathrm{Hz}, 2 \mathrm{H}), 4.44(\mathrm{~d}, \mathrm{~J}=11.6 \mathrm{~Hz}, 1 \mathrm{H}), 4.38(\mathrm{~d}, \mathrm{~J}=11.6 \mathrm{~Hz}, 1 \mathrm{H}), 4.09-4.04(\mathrm{~m}, 2 \mathrm{H}), 4.02-3.94(\mathrm{~m}, 1 \mathrm{H}), 3.81(\mathrm{~s}$, $3 \mathrm{H}), 3.41$ (td, J = 6.8, $1.0 \mathrm{~Hz}, 2 \mathrm{H}), 3.33(\mathrm{~s}, 3 \mathrm{H}), 3.26(\mathrm{dd}, \mathrm{J}=9.4,1.7 \mathrm{~Hz}, 1 \mathrm{H}), 2.46(\mathrm{~s}, 3 \mathrm{H}), 2.02-1.95(\mathrm{~m}, 1 \mathrm{H})$, 1.86-1.75 (m, 2H), 1.56-1.50 (m, 1H), $0.87(\mathrm{~s}, 9 \mathrm{H}), 0.77(\mathrm{~d}, \mathrm{~J}=6.8 \mathrm{~Hz}, 3 \mathrm{H}), 0.67(\mathrm{~d}, \mathrm{~J}=6.9 \mathrm{~Hz}, 3 \mathrm{H}), 0.068(\mathrm{~s}$, $3 \mathrm{H}), 0.062(\mathrm{~s}, 3 \mathrm{H}) ;{ }^{13} \mathrm{C}$ NMR $\left(75 \mathrm{MHz}, \mathrm{CDCl}_{3}\right) \delta 158.7,144.3,133.0,130.2,129.4,128.6,127.5,113.3,80.0$, $72.3,72.2,69.0,66.5,60.3,54.7,39.8,35.4,34.7,25.7,21.1,17.9,9.0,8.8,-3.7,-4.6$; HRMS $\mathrm{m} / \mathrm{z}$ calcd for $\left(\mathrm{M}^{+}{ }^{t} \mathrm{Bu}\right) \mathrm{C}_{27} \mathrm{H}_{41} \mathrm{O}_{7} \mathrm{SSi}: 537.2342$; found: 537.2316.

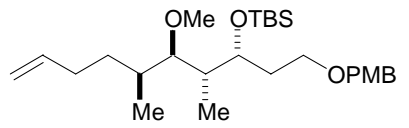

$(3 R, 4 S, 5 R, 6 S)-3-(t e r t-B u t y l d i m e t h y l s i l a n y l o x y)-5-m e t h o x y-1-(4-m e t h o x y-$ benzyloxy)-4, 6-dimethyl-9-decene (24): To a suspension of $\mathrm{CuBr}(825 \mathrm{mg}, 5.75$ mmol) in $2.5 \mathrm{~mL}$ of $\mathrm{Et}_{2} \mathrm{O}$ at $-20{ }^{\circ} \mathrm{C}$ was added allylmagnesium bromide $(1.0 \mathrm{M}$ in $\mathrm{Et}_{2} \mathrm{O}, 11.5 \mathrm{~mL}, 11.5 \mathrm{mmol}$ ) dropwise and the reaction mixture was stirred for $30 \mathrm{~min}$. To the resulting mixture was added a solution of $23(683 \mathrm{mg}, 1.15 \mathrm{mmol})$ in $4.0 \mathrm{~mL} \mathrm{Et}{ }_{2} \mathrm{O}$ and the reaction was stirred $10 \mathrm{~min}$ at $-20{ }^{\circ} \mathrm{C}$ then warmed to ambient temperature and stirred for $4.5 \mathrm{~h}$. The reaction was quenched by adding $20 \mathrm{~mL}$ of saturated aqueous $\mathrm{NH}_{4} \mathrm{Cl}$. The mixture was extracted by $\mathrm{Et}_{2} \mathrm{O}(3 \times 40 \mathrm{~mL})$ and the combined organic extracts were washed with brine, dried $\left(\mathrm{Na}_{2} \mathrm{SO}_{4}\right)$ and concentrated. The crude product mixture was purified by flash chromatography $\left(\mathrm{SiO}_{2}, 6 \%\right.$ ethyl acetate in hexane) to give $454 \mathrm{mg}(85 \%)$ of the title compound as an oil. $[\alpha]_{\mathrm{D}}$ -12.4 (c 1.65, $\mathrm{CHCl}_{3}$ ); IR (thin film): 1640, 1614, 1514, 1249, 1097, $835 \mathrm{~cm}^{-1} ;{ }^{1} \mathrm{H}$ NMR (300 MHz, $\left.\mathrm{CDCl}_{3}\right)$ $\delta 7.27(\mathrm{~d}, \mathrm{~J}=8.7 \mathrm{~Hz}, 2 \mathrm{H}), 6.88(\mathrm{~d}, \mathrm{~J}=8.7 \mathrm{~Hz}, 2 \mathrm{H}), 5.83(\mathrm{ddt}, 17.0,10.2,6.7 \mathrm{~Hz}, 1 \mathrm{H}), 5.0(\mathrm{~m}, 2 \mathrm{H}), 4.45(\mathrm{~d}, \mathrm{~J}=$ $11.5 \mathrm{~Hz}, 1 \mathrm{H}), 4.40(\mathrm{~d}, \mathrm{~J}=11.5 \mathrm{~Hz}, 1 \mathrm{H}), 4.13(\mathrm{td}, \mathrm{J}=6.9,1.4 \mathrm{~Hz}, 1 \mathrm{H}), 3.81(\mathrm{~s}, 3 \mathrm{H}), 3.47(\mathrm{~s}, 3 \mathrm{H}), 3.43(\mathrm{td}, \mathrm{J}=$ 6.8, $1.7 \mathrm{~Hz}, 2 \mathrm{H}), 3.13(\mathrm{dd}, \mathrm{J}=9.4,1.7 \mathrm{~Hz}, 1 \mathrm{H}), 2.23-2.02(\mathrm{~m}, 2 \mathrm{H}), 1.89-1.82(\mathrm{~m}, 2 \mathrm{H}), 1.69-1.40(\mathrm{~m}, 4 \mathrm{H}), 0.89$ $(\mathrm{s}, 9 \mathrm{H}), 0.83(\mathrm{~d}, \mathrm{~J}=6.7 \mathrm{~Hz}, 3 \mathrm{H}), 0.73(\mathrm{~d}, \mathrm{~J}=6.9 \mathrm{~Hz}, 3 \mathrm{H}), 0.091(\mathrm{~s}, 3 \mathrm{H}), 0.083(\mathrm{~s}, 3 \mathrm{H}) ;{ }^{13} \mathrm{C} \mathrm{NMR}(75 \mathrm{MHz}$, $\left.\mathrm{CDCl}_{3}\right) \delta 159.0,139.0,130.6,129.1,114.3,113.7,84.7,72.6,69.3,67.2,60.8,55.2,40.7,35.9,34.4,32.0,26.0$, 18.3, 12.8, 9.5, -3.4,-4.3; HRMS m/z calcd for $\mathrm{C}_{27} \mathrm{H}_{48} \mathrm{O}_{4} \mathrm{Si}$ : 464.3322 ; found: 464.3304 .

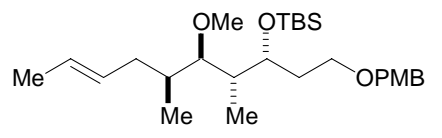

$(E, \quad 3 R, \quad 4 S, \quad 5 R, \quad 6 S)-3$-(tert-Butyldimethylsilanyloxy)-5-methoxy-1-(4methoxybenzyloxy)-4, 6-dimethyl-8-decene (25): To a solution of $\mathrm{NaBPh}_{4}(7.7$ $\mathrm{mg}, 0.0225 \mathrm{mmol})$ in $0.2 \mathrm{~mL}$ of acetone was added a solution of $\left[\left({ }^{c} \mathrm{C}_{8} \mathrm{H}_{14}\right)_{2} \mathrm{IrCl}\right]_{2}$ (10.2 $\mathrm{mg}, 0.0113 \mathrm{mmol}$ ) and $\mathrm{PCy}_{3}(19.0 \mathrm{mg}, 0.0678 \mathrm{mmol})$ in $10.8 \mathrm{~mL}$ of $\mathrm{CH}_{2} \mathrm{Cl}_{2}$. The reaction mixture was stirred for $5 \mathrm{~min}$, then this catalyst solution was added to $24(550 \mathrm{mg}, 1.185 \mathrm{mmol})$. The reaction mixture was stirred for $3 \mathrm{~h}$ and then filtered through silica gel eluting with $120 \mathrm{~mL}$ of $\mathrm{Et}_{2} \mathrm{O}$. The filtrate was concentrated and the crude product mixture was purified by flash chromatography $\left(\mathrm{SiO}_{2}, 6 \%\right.$ ethyl acetate in hexane) to give $539 \mathrm{mg}(98 \%)$ of the title compound as a colorless oil. $[\alpha]_{\mathrm{D}}-8.5$ (c 1.02, $\left.\mathrm{CHCl}_{3}\right)$; IR (thin film): 1614, 1249, 1097, $835 \mathrm{~cm}^{-1} ;{ }^{1} \mathrm{H}$ NMR $\left(300 \mathrm{MHz}, \mathrm{CDCl}_{3}\right) \delta 7.27(\mathrm{~d}, \mathrm{~J}=8.7 \mathrm{~Hz}, 2 \mathrm{H}), 6.88(\mathrm{~d}, \mathrm{~J}=8.7 \mathrm{~Hz}, 2 \mathrm{H}), 5.51-5.36(\mathrm{~m}$, $2 \mathrm{H}), 4.45(\mathrm{~d}, \mathrm{~J}=11.6 \mathrm{~Hz}, 1 \mathrm{H}), 4.40(\mathrm{~d}, \mathrm{~J}=11.6 \mathrm{~Hz}, 1 \mathrm{H}), 4.12(\mathrm{t}, \mathrm{J}=6.2 \mathrm{~Hz}, 1 \mathrm{H}), 3.81(\mathrm{~s}, 3 \mathrm{H}), 3.48(\mathrm{~s}, 3 \mathrm{H}), 3.43$ (td, J = 7.7, $2.4 \mathrm{~Hz}, 2 \mathrm{H}), 3.18(\mathrm{dd}, \mathrm{J}=9.4,1.6 \mathrm{~Hz}, 1 \mathrm{H}), 2.15(\mathrm{~m}, 1 \mathrm{H}), 2.01(\mathrm{~m}, 1 \mathrm{H}), 1.87(\mathrm{dd}, \mathrm{J}=6.8,2.2 \mathrm{~Hz}$, $1 \mathrm{H}), 1.82(\mathrm{dd}, \mathrm{J}=7.3,3.6 \mathrm{~Hz}, 1 \mathrm{H}), 1.67(\mathrm{~d}, \mathrm{~J}=5.0 \mathrm{~Hz}, 3 \mathrm{H}), 1.58(\mathrm{br}, 1 \mathrm{H}), 1.54-1.46(\mathrm{~m}, 1 \mathrm{H}), 0.89(\mathrm{~s}, 9 \mathrm{H}), 0.81$ $(\mathrm{d}, \mathrm{J}=6.8 \mathrm{~Hz}, 3 \mathrm{H}), 0.73(\mathrm{~d}, \mathrm{~J}=6.9 \mathrm{~Hz}, 3 \mathrm{H}), 0.079(\mathrm{~s}, 6 \mathrm{H}) ;{ }^{13} \mathrm{C} \mathrm{NMR}\left(75 \mathrm{MHz}, \mathrm{CDCl}_{3}\right) \delta 158.9,130.5,128.9$, 
126.0, 113.5, 83.6, 72.4, 69.3, 67.0, 60.6, 54.9, 40.7, 38.2, 35.9, 35.5, 25.9, 18.2, 17.8, 12.7, 9.4, -3.5, -4.5; HRMS m/z calcd for $\mathrm{C}_{27} \mathrm{H}_{48} \mathrm{O}_{4} \mathrm{Si}: 464.3322$; found: 464.3345 .

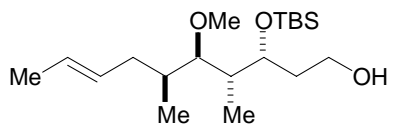

$(E, \quad 3 R, \quad 4 S, \quad 5 R, \quad 6 S)-3-(t e r t-B u t y l d i m e t h y l s i l a n y l o x y)-5-m e t h o x y-4, \quad 6-$ dimethyldec-8-en-1-ol (25'): To a $0{ }^{\circ} \mathrm{C}$ solution of DDQ (282 mg, $\left.1.24 \mathrm{mmol}\right)$ in 2.2 $\mathrm{mL}$ of $\mathrm{CH}_{2} \mathrm{Cl}_{2}$ and $0.45 \mathrm{~mL}$ of $\mathrm{pH} 7$ buffer was added a solution of 25 (486 mg, 1.05 mmol) in $7.0 \mathrm{~mL}$ of $\mathrm{CH}_{2} \mathrm{Cl}_{2}$. The resulting mixture was stirred for $40 \mathrm{~min}$ and then quenched by adding $10 \mathrm{~mL}$ of $\mathrm{H}_{2} \mathrm{O}$. The mixture was extracted with $\mathrm{Et}_{2} \mathrm{O}(3 \times 40 \mathrm{~mL})$ and the combined organic extracts were washed with brine, dried $\left(\mathrm{Na}_{2} \mathrm{SO}_{4}\right)$ and concentrated. The crude product mixture was purified by flash chromatography $\left(\mathrm{SiO}_{2}\right.$, gradient $6 \% \rightarrow 10 \% \rightarrow 20 \%$ ethyl acetate in hexane) to give $291 \mathrm{mg}(81 \%)$ of the title compound as an oil. $[\alpha]_{\mathrm{D}}-9.3$ (c 1.40, $\mathrm{CHCl}_{3}$ ); IR (thin film): 3401(br), 1253, 1077, 835, $774 \mathrm{~cm}^{-1} ;{ }^{1} \mathrm{H}$ NMR (300 MHz, $\left.\mathrm{CDCl}_{3}\right)$ $\delta$ 5.51-5.35 (m, 2H), 4.15-4.10 (m, 1H), $3.69(\mathrm{td}, \mathrm{J}=6.5,1.4 \mathrm{~Hz}, 2 \mathrm{H}), 3.49(\mathrm{~s}, 3 \mathrm{H}), 3.16(\mathrm{dd}, \mathrm{J}=9.2,1.8 \mathrm{~Hz}$, $1 \mathrm{H}), 2.15(\mathrm{~m}, 1 \mathrm{H}), 2.01(\mathrm{~m}, 1 \mathrm{H}), 1.84-1.76(\mathrm{~m}, 2 \mathrm{H}), 1.67(\mathrm{~d}, \mathrm{~J}=5.3 \mathrm{~Hz}, 3 \mathrm{H}), 1.64-1.59(\mathrm{~m}, 2 \mathrm{H}), 0.91(\mathrm{~s}, 9 \mathrm{H})$, $0.83(\mathrm{~d}, \mathrm{~J}=6.8 \mathrm{~Hz}, 3 \mathrm{H}), 0.77(\mathrm{~d}, \mathrm{~J}=7.0 \mathrm{~Hz}, 3 \mathrm{H}), 0.113(\mathrm{~s}, 3 \mathrm{H}), 0.107(\mathrm{~s}, 3 \mathrm{H}) ;{ }^{13} \mathrm{C} \mathrm{NMR}\left(75 \mathrm{MHz}, \mathrm{CDCl}_{3}\right)$ $\delta 130.3,126.3,84.2,69.2,60.8,59.4,40.2,38.5,38.2,35.5,25.9,18.2,17.9,12.6,9.6,-3.4,-4.4 ; \mathrm{HRMS} \mathrm{m} / \mathrm{z}$ calcd for $\left(\mathrm{M}^{+}-{ }^{t} \mathrm{Bu}\right) \mathrm{C}_{15} \mathrm{H}_{31} \mathrm{O}_{3} \mathrm{Si}: 287.2042$; found: 287.2050 .

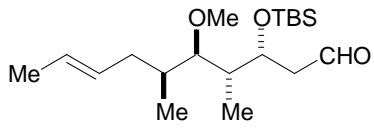

(E, 3R, 4S, 5R, 6S)-3-(tert-Butyldimethylsilanyloxy)-5-methoxy-4, 6-dimethyldec8-enal (26): To a solution of DMSO (139 uL, $1.96 \mathrm{mmol})$ in $5.0 \mathrm{~mL}$ of $\mathrm{CH}_{2} \mathrm{Cl}_{2}$ at -78 ${ }^{\circ} \mathrm{C}$ was added a solution of $(\mathrm{COCl})_{2}(1.25$ equiv, $490 \mu \mathrm{L}, 0.98 \mathrm{mmol})$ in $\mathrm{CH}_{2} \mathrm{Cl}_{2}(0.5$ $\mathrm{mL}$ ) dropwise. The reaction mixture was stirred for $40 \mathrm{~min}$ whereupon a solution of $\mathbf{2 5}^{\prime}(270 \mathrm{mg}, 0.785 \mathrm{mmol})$ in $2.5 \mathrm{~mL}$ of $\mathrm{CH}_{2} \mathrm{Cl}_{2}$ was added. The resulting mixture was stirred for $30 \mathrm{~min}$ then $\mathrm{Et}_{3} \mathrm{~N}$ (548 uL, $3.92 \mathrm{mmol}$ ) was added dropwise and the reaction mixture was stirred for another $30 \mathrm{~min}$ then warmed to ambient temperature and quenched by adding $10 \mathrm{~mL}$ of saturated aqueous $\mathrm{NH}_{4} \mathrm{Cl}$. The mixture was extracted by $\mathrm{CH}_{2} \mathrm{Cl}_{2}$ $(3 \times 40 \mathrm{~mL})$ and the combined organic extracts were washed with brine, dried $\left(\mathrm{Na}_{2} \mathrm{SO}_{4}\right)$ and concentrated. The crude product mixture was purified by flash chromatography $\left(\mathrm{SiO}_{2}, 6 \%\right.$ ethyl acetate in hexane) to give $235 \mathrm{mg}$ (88\%) of the title compound as an oil. $[\alpha]_{\mathrm{D}}-6.2$ (c 1.62, $\mathrm{CHCl}_{3}$ ); IR (thin film): $2718,1728,1472,1134,1089$, 836, $775 \mathrm{~cm}^{-1}$; ${ }^{1} \mathrm{H}$ NMR $\left(300 \mathrm{MHz}, \mathrm{CDCl}_{3}\right) \delta 9.79(\mathrm{t}, \mathrm{J}=2.4 \mathrm{~Hz}, 1 \mathrm{H}), 5.53-5.35(\mathrm{~m}, 2 \mathrm{H}), 4.63(\mathrm{td}, \mathrm{J}=6.2,1.8$ $\mathrm{Hz}, 1 \mathrm{H}), 3.50(\mathrm{~s}, 3 \mathrm{H}), 3.17(\mathrm{dd}, \mathrm{J}=9.2,1.8 \mathrm{~Hz}, 1 \mathrm{H}), 2.67(\mathrm{ddd}, \mathrm{J}=16.1,6.2,2.5 \mathrm{~Hz}, 1 \mathrm{H}), 2.55(\mathrm{ddd}, \mathrm{J}=16.1$, 6.2, $2.3 \mathrm{~Hz}, 1 \mathrm{H}), 2.15(\mathrm{~m}, 1 \mathrm{H}), 2.05(\mathrm{~m}, 1 \mathrm{H}), 1.76-1.66(\mathrm{~m}, 1 \mathrm{H}), 1.67(\mathrm{~d}, \mathrm{~J}=4.9 \mathrm{~Hz}, 3 \mathrm{H}), 1.60-1.50(\mathrm{~m}, 1 \mathrm{H})$, $0.89(\mathrm{~s}, 9 \mathrm{H}), 0.82(\mathrm{~d}, \mathrm{~J}=6.9 \mathrm{~Hz}, 3 \mathrm{H}), 0.80(\mathrm{~d}, \mathrm{~J}=7.0 \mathrm{~Hz}, 3 \mathrm{H}), 0.13(\mathrm{~s}, 3 \mathrm{H}), 0.07(\mathrm{~s}, 3 \mathrm{H}) ;{ }^{13} \mathrm{C} \mathrm{NMR}(75 \mathrm{MHz}$, $\left.\mathrm{CDCl}_{3}\right) \delta 201.1,130.3,126.3,83.9,66.9,60.6,50.8,42.6,38.2,35.5,25.8,18.2,17.9,12.8,10.1,-3.8,-4.5$; HRMS (electrospray) $\mathrm{m} / \mathrm{z}$ calcd for $\mathrm{C}_{19} \mathrm{H}_{38} \mathrm{O}_{3} \mathrm{NaSi}(\mathrm{M}+\mathrm{Na})^{+}: 365.2488$; found: 365.2473 .

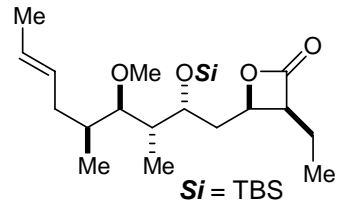

$(3 S, 4 R)-4-[(E, 2 R, 3 S, 4 R, 5 S)-2-(t e r t-B u t y l d i m e t h y l s i l a n y l o x y)-4-m e t h o x y-3,5-$ dimethylnon-7-enyl]-3-ethyloxetan-2-one (28): To a solution of triamine ligand (169.5 $\mathrm{mg}, 0.25 \mathrm{mmol})^{4}$ in $2.0 \mathrm{~mL}$ of benzotrifluoride at ambient temperature was slowly added a solution of $\mathrm{AlMe}_{3}(2.0 \mathrm{M}$ in hexane, $0.125 \mathrm{~mL}, 0.25 \mathrm{mmol})$. After stirring for 2 $\mathrm{h}$, the resulting catalyst solution was cooled to $-25^{\circ} \mathrm{C}$ and $N, N$-diisopropylethylamine $(0.173 \mathrm{~mL}, 1.0 \mathrm{mmol})$ and butyryl bromide $(0.212 \mathrm{~mL}, 2.0 \mathrm{mmol})$ were added consecutively. The resulting heterogeneous mixture was stirred $20 \mathrm{~min}$ at $-25{ }^{\circ} \mathrm{C}$ whereupon $26(170 \mathrm{mg}, 0.50 \mathrm{mmol})$ in $1.0 \mathrm{~mL}$ of benzotrifluoride was added dropwise and the reaction was stirred $21 \mathrm{~h}$ at $-25^{\circ} \mathrm{C}$. The reaction was quenched at $-25{ }^{\circ} \mathrm{C}$ by adding $10 \mathrm{~mL}$ of a $3 \% \mathrm{Et}_{3} \mathrm{~N}-\mathrm{Et}_{2} \mathrm{O}$ solution $(\mathrm{v} / \mathrm{v})$ and the resulting mixture was filtered through silica gel eluting with $60 \mathrm{~mL}$ of $\mathrm{Et}_{2} \mathrm{O}$. The filtrate was concentrated and the crude product mixture was purified by flash chromatography (gradient $4 \% \rightarrow 6 \%$ ethyl acetate in hexane) to give $135 \mathrm{mg}(65 \%)$ of the title compound. $[\alpha]_{\mathrm{D}}+20.2$ (c 1.06, $\mathrm{CHCl}_{3}$ ); IR (thin film): 1828, 1462, 1254, 836, $775 \mathrm{~cm}^{-1} ;{ }^{1} \mathrm{H}$ NMR $\left(300 \mathrm{MHz}, \mathrm{CDCl}_{3}\right) \delta$ 5.53-5.35 (m, 2H), $4.64(\mathrm{dt}, \mathrm{J}=6.5,6.5 \mathrm{~Hz}, 1 \mathrm{H}), 4.20(\mathrm{td}, \mathrm{J}=6.9,1.1 \mathrm{~Hz}, 1 \mathrm{H}), 3.57(\mathrm{dt}, \mathrm{J}=9.1,7.4 \mathrm{~Hz}, 1 \mathrm{H})$, $3.48(\mathrm{~s}, 3 \mathrm{H}), 3.16(\mathrm{dd}, \mathrm{J}=9.5,1.5 \mathrm{~Hz}, 1 \mathrm{H}), 2.15(\mathrm{~m}, 1 \mathrm{H}), 2.02(\mathrm{~m}, 1 \mathrm{H}), 1.89(\mathrm{t}, \mathrm{J}=6.5 \mathrm{~Hz}, 2 \mathrm{H}), 1.85-1.79(\mathrm{~m}$, 1H), 1.74-1.66 (m, 1H), $1.67(\mathrm{~d}, \mathrm{~J}=4.7 \mathrm{~Hz}, 3 \mathrm{H}), 1.62-1.60(\mathrm{~m}, 2 \mathrm{H}), 1.09(\mathrm{t}, \mathrm{J}=7.4 \mathrm{~Hz}, 3 \mathrm{H}), 0.91(\mathrm{~s}, 9 \mathrm{H}), 0.82$ $(\mathrm{d}, \mathrm{J}=6.8 \mathrm{~Hz}, 3 \mathrm{H}), 0.76(\mathrm{~d}, \mathrm{~J}=6.9 \mathrm{~Hz}, 3 \mathrm{H}), 0.11(\mathrm{~s}, 3 \mathrm{H}), 0.10(\mathrm{~s}, 3 \mathrm{H}) ;{ }^{13} \mathrm{C} \mathrm{NMR}\left(75 \mathrm{MHz}, \mathrm{CDCl}_{3}\right) \delta 172.0$, 130.4, 126.4, 83.8, 72.6, 69.2, 60.8, 54.2, 41.6, 38.3, 36.6, 35.6, 25.9, 18.3, 18.0, 17.6, 12.7, 12.1, 9.9, -3.7, -3.9; HRMS (electrospray) $\mathrm{C}_{23} \mathrm{H}_{44} \mathrm{O}_{4} \mathrm{NaSi}(\mathrm{M}+\mathrm{Na})^{+}: 435.2907$; found: 435.2897 . 


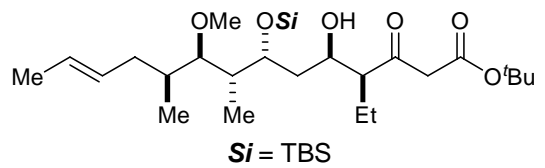

$(E, 4 S, 5 R, 7 R, 8 S, 9 R, 10 S)$-tert-Butyl 7-(tert-butyldimethylsilanyloxy)-4ethyl-5-hydroxy-9-methoxy-8, 10-dimethyl-3-oxotetradec-12-enoate (29): To a solution of KHMDS $(0.5 \mathrm{M}$ in toluene, $1.14 \mathrm{~mL}, 0.57 \mathrm{mmol})$ in $1.0 \mathrm{~mL}$ of $\mathrm{THF}$ at $-78{ }^{\circ} \mathrm{C}$ was added tert-butyl acetate $(66.2 \mathrm{mg}, 0.57$ $\mathrm{mmol})$. The reaction mixture was stirred for $1 \mathrm{~h}$ then transferred via cannula to a suspension of $\mathrm{MgBr}_{2} \cdot \mathrm{OEt}_{2}(147$ $\mathrm{mg}, 0.57 \mathrm{mmol}$ ) in $1.0 \mathrm{~mL}$ of THF. The resulting mixture was stirred for $1 \mathrm{~h}$ whereupon 28 (47 mg, 0.114 $\mathrm{mmol}$ ) was added and the reaction was stirred for an additional $30 \mathrm{~min}$. The reaction was quenched by adding $10 \mathrm{~mL}$ of saturated aqueous $\mathrm{NH}_{4} \mathrm{Cl}$ and the mixture was extracted with $\mathrm{Et}_{2} \mathrm{O}(3 \times 30 \mathrm{~mL})$. The combined organic extracts were washed with brine, dried $\left(\mathrm{Na}_{2} \mathrm{SO}_{4}\right)$ and concentrated. The crude product mixture was purified by flash chromatography $\left(\mathrm{SiO}_{2}, 15 \%\right.$ ethyl acetate in hexanes) to give $40 \mathrm{mg}(66 \%)$ of the title compound as an inseperatable mixture keto-enol tautomers. IR (thin film): 3467 (br), $1733,1709 \mathrm{~cm}^{-1} ;{ }^{1} \mathrm{H} \mathrm{NMR}$ $\left(300 \mathrm{MHz}_{\mathrm{CDCl}}\right) \delta 5.50-5.33(\mathrm{~m}, 2 \mathrm{H}), 4.14-4.10(\mathrm{~m}, 1 \mathrm{H}), 4.04(\mathrm{q}, \mathrm{J}=4.9 \mathrm{~Hz}, 1 \mathrm{H}), 3.48(\mathrm{~d}, \mathrm{~J}=1.6 \mathrm{~Hz}, 2 \mathrm{H})$, $3.44(\mathrm{~s}, 3 \mathrm{H}), 3.06(\mathrm{dd}, \mathrm{J}=8.2,1.9 \mathrm{~Hz}, 1 \mathrm{H}), 2.75(\mathrm{dt}, \mathrm{J}=8.9,4.3 \mathrm{~Hz}, 1 \mathrm{H}), 2.16-2.07(\mathrm{~m}, 1 \mathrm{H}), 2.02-1.93(\mathrm{~m}, 1 \mathrm{H})$, 1.88-1.73 (m, 3H), $1.67(\mathrm{~d}, \mathrm{~J}=5.2 \mathrm{~Hz}, 3 \mathrm{H}), 1.63-1.58(\mathrm{~m}, 4 \mathrm{H}), 1.47(\mathrm{~s}, 9 \mathrm{H}), 0.92(\mathrm{t}, \mathrm{J}=7.4 \mathrm{~Hz}, 3 \mathrm{H}), 0.90(\mathrm{~s}$, $9 \mathrm{H}), 0.85(\mathrm{~d}, \mathrm{~J}=6.8 \mathrm{~Hz}, 3 \mathrm{H}), 0.84(\mathrm{~d}, \mathrm{~J}=6.9 \mathrm{~Hz}, 3 \mathrm{H}), 0.11(\mathrm{~s}, 3 \mathrm{H}), 0.10(\mathrm{~s}, 3 \mathrm{H}) ;{ }^{13} \mathrm{C} \mathrm{NMR}\left(75 \mathrm{MHz}^{\mathrm{N}} \mathrm{CDCl}\right)_{3} \delta$ 206.6, 166.5, 130.2, 126.5, 84.7, 81.7, 71.6, 69.3, 60.2, 59.0, 52.0, 40.7, 39.2, 38.6, 35.5, 28.3, 28.0, 26.0, 19.8, 17.9, 13.4, 12.2, 11.5, -3.8, -4.3; HRMS m/z calcd for $\mathrm{C}_{29} \mathrm{H}_{56} \mathrm{O}_{6} \mathrm{Si}$ : 528.3846; found: 528.3842.

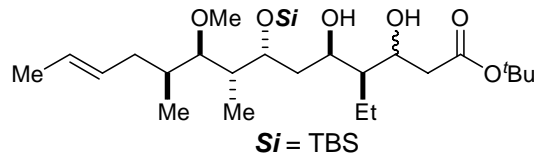

$(E, 4 S, 5 R, 7 R, 8 S, 9 R, 10 S)$-tert-Butyl 4-ethyl-3,5,7-trihydroxy-9-methoxy8, 10-dimethyltetradec-12-enoate (29'): To a solution of 29 (27 $\mathrm{mg}, 0.05$ $\mathrm{mmol})$ in $1.0 \mathrm{~mL}$ of EtOH at $0{ }^{\circ} \mathrm{C}$ was added $\mathrm{NaBH}_{4}(6 \mathrm{mg}, 0.16 \mathrm{mmol})$. The resulting mixture was stirred for $40 \mathrm{~min}$ and then quenched by adding $50 \mu \mathrm{L}$ acetic acid. The mixture was extracted with ethyl acetate $(3 \times 30 \mathrm{~mL})$ and the combined organic extracts were washed with brine, dried $\left(\mathrm{Na}_{2} \mathrm{SO}_{4}\right)$ and concentrated. The crude product used without further purification.

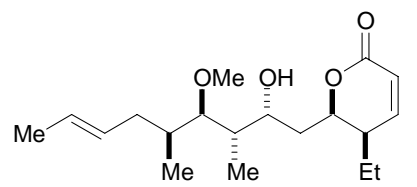

(-)-Pironetin (19): A solution of 29' (15 $\mathrm{mg}$ crude product from previous transformation) and $\mathrm{TsOH} \cdot \mathrm{H}_{2} \mathrm{O}(5 \mathrm{mg})$ in $1.0 \mathrm{~mL}$ of toluene was heated at $110{ }^{\circ} \mathrm{C}$ for $30 \mathrm{~min}$. The mixture was then cooled to ambient temperature and diluted with $50 \mathrm{~mL}$ of ethyl acetate. The resulting mixture was washed successively with $10 \mathrm{~mL}$ of aqueous $\mathrm{NaHCO}_{3}$ solution and brine and the organic layer was dried $\left(\mathrm{Na}_{2} \mathrm{SO}_{4}\right)$ and concentrated. The crude product mixture was purified by flash chromatography $\left(\mathrm{SiO}_{2}, 40 \%\right.$ ethyl acetate in hexanes) to give $4.6 \mathrm{mg}$ (56\% over two steps) of the title compound as a white solid. $[\alpha]_{\mathrm{D}}-135\left(c 0.35, \mathrm{CHCl}_{3}\right)\left\{\right.$ lit. $^{10}[\alpha]_{\mathrm{D}}-144(c 0.50$, $\left.\left.\mathrm{CHCl}_{3}\right)\right\} ;{ }^{1} \mathrm{H}$ NMR $\left(300 \mathrm{MHz}, \mathrm{CDCl}_{3}\right) \delta 7.02(\mathrm{dd}, \mathrm{J}=9.8,6.0 \mathrm{~Hz}, 1 \mathrm{H}), 6.03(\mathrm{~d}, \mathrm{~J}=9.8 \mathrm{~Hz}, 1 \mathrm{H}), 5.51-5.32(\mathrm{~m}$, 2H), $4.74(\mathrm{~m}, 1 \mathrm{H}), 4.21$ (brd, 1H), $3.48(\mathrm{~s}, 3 \mathrm{H}), 3.45(\mathrm{~d}, \mathrm{~J}=2.3 \mathrm{~Hz}, 1 \mathrm{H}), 2.99$ (dd, J = 6.1, 4.4 Hz, 1H), 2.33$2.25(\mathrm{~m}, 1 \mathrm{H}), 2.13-2.06(\mathrm{~m}, 1 \mathrm{H}), 1.92-1.84(\mathrm{~m}, 2 \mathrm{H}), 1.81-1.76(\mathrm{~m}, 1 \mathrm{H}), 1.74-1.65(\mathrm{~m}, 3 \mathrm{H}), 1.67(\mathrm{~d}, \mathrm{~J}=5.3 \mathrm{~Hz}$, $3 \mathrm{H}), 1.58-1.46(\mathrm{~m}, 1 \mathrm{H}), 1.01(\mathrm{~d}, \mathrm{~J}=7.1 \mathrm{~Hz}, 3 \mathrm{H}), 0.97(\mathrm{t}, \mathrm{J}=7.4 \mathrm{~Hz}, 3 \mathrm{H}), 0.96(\mathrm{~d}, \mathrm{~J}=6.9 \mathrm{~Hz}, 3 \mathrm{H}) ;{ }^{13} \mathrm{C}$ NMR $(75$ $\left.\mathrm{MHz}, \mathrm{CDCl}_{3}\right) \delta 164.6,150.6,128.8,126.9,120.9,91.1,77.8,67.5,61.5,39.1,39.0,37.3,36.7,36.2,20.8,17.9$, 15.2, 12.2, 11.0; HRMS $m / z$ calcd for $\left(\mathrm{M}^{+}-\mathrm{H}_{2} \mathrm{O}\right) \mathrm{C}_{19} \mathrm{H}_{30} \mathrm{O}_{3}: 306.2195$; found: 306.2185 .

\footnotetext{
${ }^{10}$ Kobayashi, S.; Tsochia, K.; Harada, T.; Nishide, M.; Kurokawa, T.; Nakagawa, T.; Shimada, N.; Kobayashi, K. J. Antibiot. 1994, 47, 697.
} 


\section{Stereochemical Proofs}

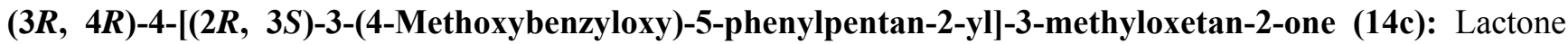
$14 \mathbf{c}$ was converted to the crystalline derivative $14 \mathbf{c}^{\prime}$ whose structure was determined by $\mathrm{X}$-ray diffraction analysis.

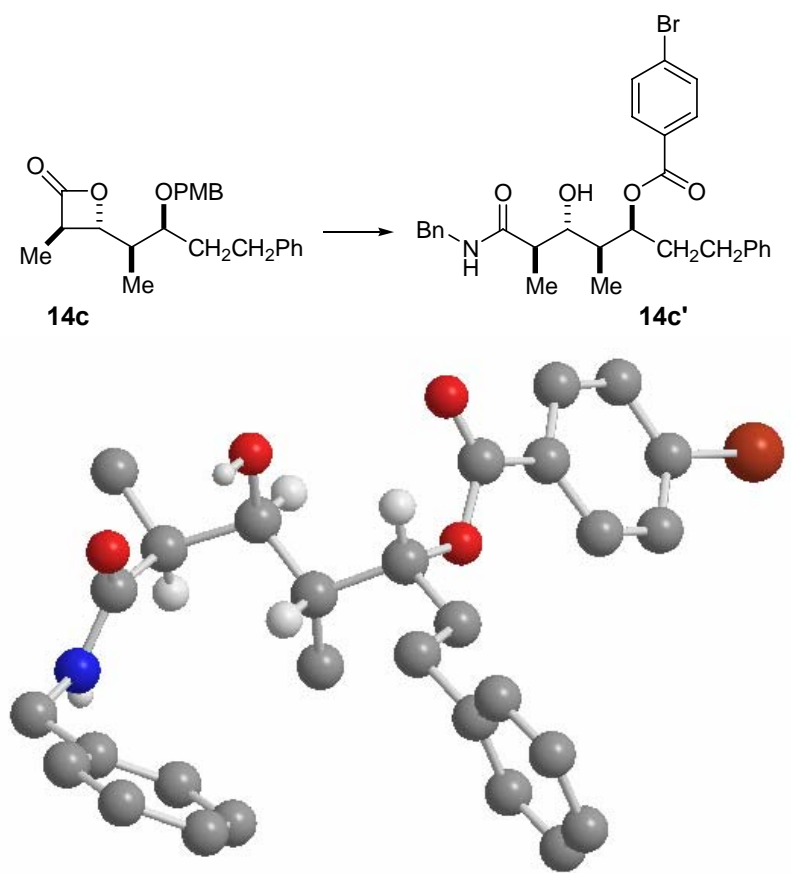

Figure S1. X-ray Crystal Structure of (3S,4S,5R,6R)-6-(Benzylcarbamoyl)-5-hydroxy-4-methyl-1-phenylheptan-3-yl 4-bromobenzoate (14c')

Table S1. Crystal data and structure refinement for $14 \mathbf{c}^{\prime}$

Empirical formula

Formula weight

Temperature

Wavelength

Crystal system

Space group

Unit cell dimensions

Volume

Z

Density (calculated)

Absorption coefficient

$\mathrm{F}(000)$

Theta range for data collection

Index ranges

Reflections collected

Independent reflections

Completeness to theta $=25.00^{\circ}$

Absorption correction

Refinement method

Data / restraints / parameters

Goodness-of-fit on $\mathrm{F}^{2}$

Final $\mathrm{R}$ indices [I $>2 \operatorname{sigma}(\mathrm{I})]$

$\mathrm{R}$ indices (all data)

Absolute structure parameter

Largest diff. peak and hole
$\mathrm{C}_{29} \mathrm{H}_{32} \mathrm{BrNO}_{4}$

538.47

$295(2){ }^{\circ} \mathrm{K}$

$0.71073 \AA$

Monoclinic

P2(1)

$\mathrm{a}=7.3752(8) \AA$

$\alpha=90^{\circ}$.

$\mathrm{b}=7.6119(8) \AA$

$\beta=92.553(2)^{\circ}$.

$\mathrm{c}=24.195(3) \AA$

$\gamma=90^{\circ}$.
2

$1.318 \mathrm{Mg} / \mathrm{m}^{3}$

$1.548 \mathrm{~mm}^{-1}$

560

2.53 to $25.00^{\circ}$.

$-8<=\mathrm{h}<=8,0<=\mathrm{k}<=9,0<=\mathrm{l}<=28$

2579

$2579[\mathrm{R}(\mathrm{int})=0.0000]$

$99.9 \%$

None

Full-matrix least-squares on $\mathrm{F}^{2}$

$2579 / 1 / 287$

0.851

$\mathrm{R} 1=0.0604, \mathrm{wR} 2=0.1269$

$\mathrm{R} 1=0.1123, \mathrm{wR} 2=0.1383$

$0.39(5)$

0.980 and -0.228 e. $\AA^{-3}$ 


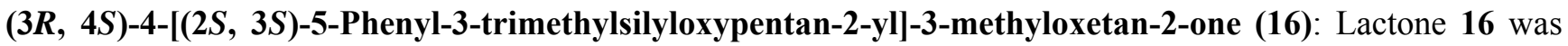
converted to the crystalline derivative $\mathbf{1 6}^{\prime}$ ' whose structure was determined by X-ray diffraction analysis.

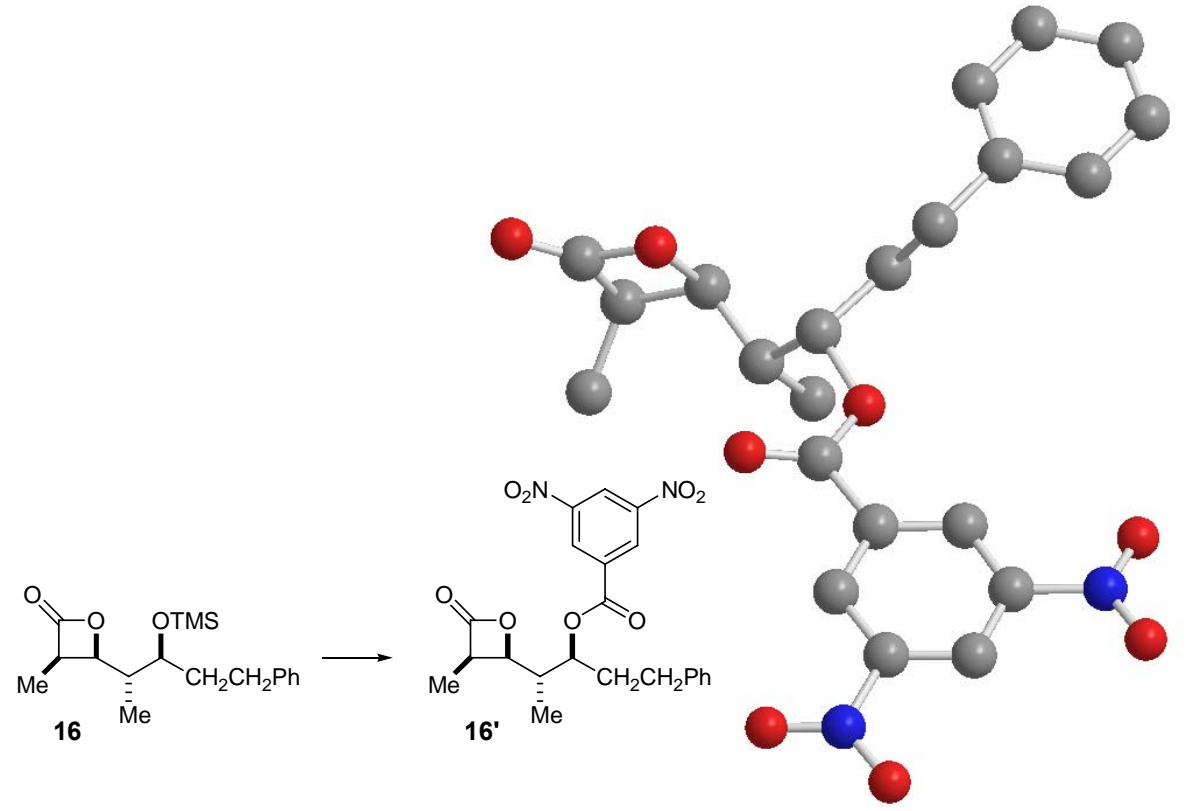

Figure S2. X-ray Crystal Structure of (3S,4S)-4-[(2S,3R)-3-methyl-4-oxo-2-oxetanyl] -1-phenylpentan-3-yl 3,5-dinitrobenzoate (16')

Table S2. Crystal data and structure refinement for 16'

\begin{tabular}{|c|c|c|}
\hline Empirical formula & $\mathrm{C}_{22} \mathrm{H}_{22} \mathrm{~N}_{2} \mathrm{O}_{8}$ & \\
\hline Formula weight & 442.42 & \\
\hline Temperature & 295(2) K & \\
\hline Wavelength & $0.71073 \AA$ & \\
\hline Crystal system & Orthorhombic & \\
\hline Space group & $\mathrm{P} 2(1) 2(1) 2(1)$ & \\
\hline Unit cell dimensions & $\mathrm{a}=7.3533(5) \AA$ & $\alpha=90^{\circ}$. \\
\hline & $\mathrm{b}=10.3188(8) \AA$ & $\beta=90^{\circ}$. \\
\hline & $\mathrm{c}=27.986(2) \AA$ & $\gamma=90^{\circ}$. \\
\hline Volume & $2123.5(3) \AA^{3}$ & \\
\hline $\mathrm{Z}$ & 4 & \\
\hline Density (calculated) & $1.384 \mathrm{Mg} / \mathrm{m}^{3}$ & \\
\hline Absorption coefficient & $0.107 \mathrm{~mm}^{-1}$ & \\
\hline $\mathrm{F}(000)$ & 928 & \\
\hline Theta range for data collection & 1.46 to $32.56^{\circ}$. & \\
\hline Index ranges & $-11<=\mathrm{h}<=10,-15<$ & $1<=1<=42$ \\
\hline Reflections collected & 27932 & \\
\hline Independent reflections & $7574[\mathrm{R}($ int $)=0.03$ & \\
\hline Completeness to theta $=32.56^{\circ}$ & $99.3 \%$ & \\
\hline Absorption correction & None & \\
\hline Refinement method & Full-matrix least-s & \\
\hline Data / restraints / parameters & 7574 / 0 / 377 & \\
\hline Goodness-of-fit on $\mathrm{F}^{2}$ & 1.056 & \\
\hline Final $R$ indices $[\mathrm{I}>2 \operatorname{sigma}(\mathrm{I})]$ & $\mathrm{R} 1=0.0613, \mathrm{wR} 2$ & \\
\hline $\mathrm{R}$ indices (all data) & $\mathrm{R} 1=0.0874, \mathrm{wR} 2$ & \\
\hline Absolute structure parameter & $0.2(10)$ & \\
\hline Largest diff. peak and hole & 0.336 and $-0.198 \mathrm{e}$ & \\
\hline
\end{tabular}




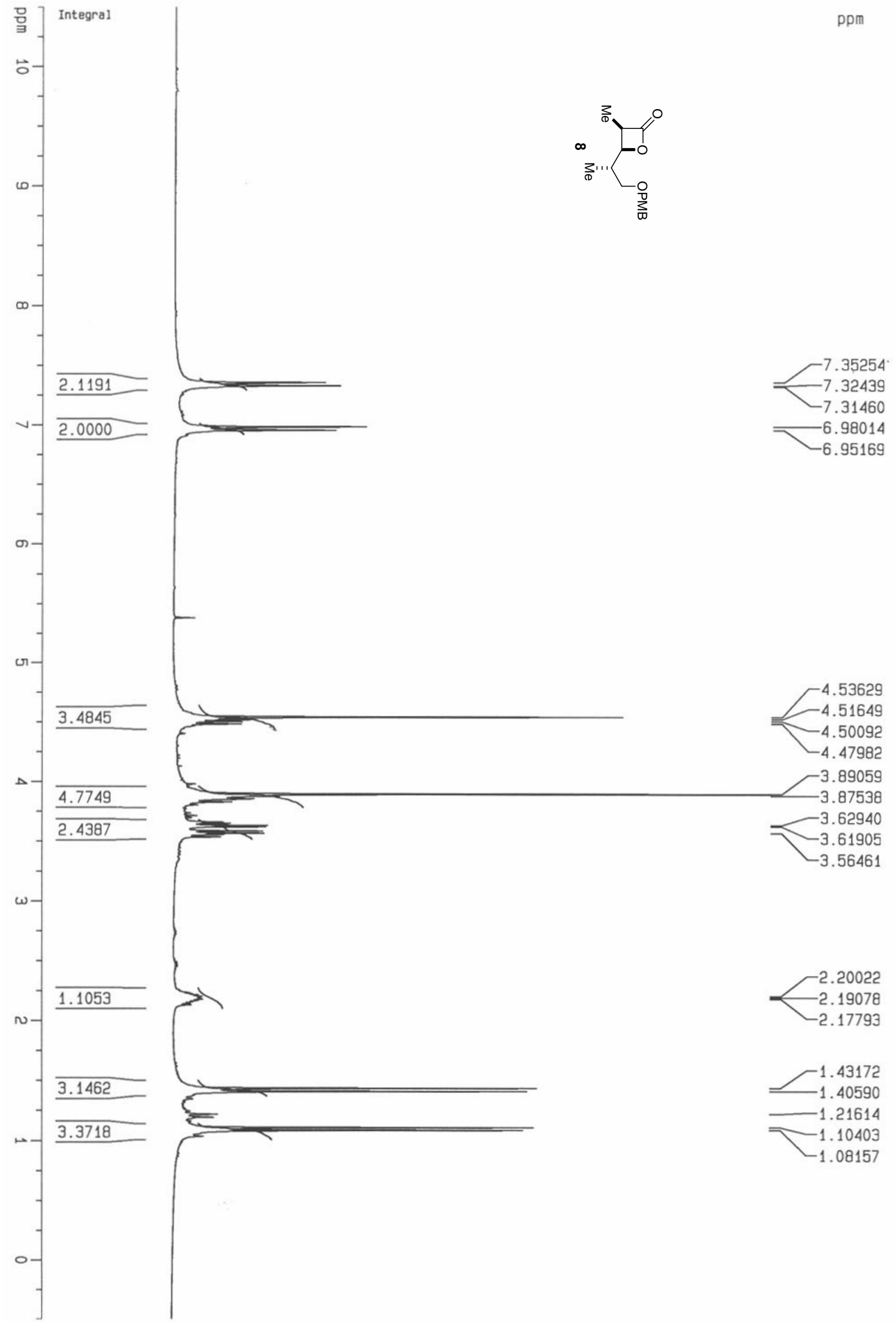




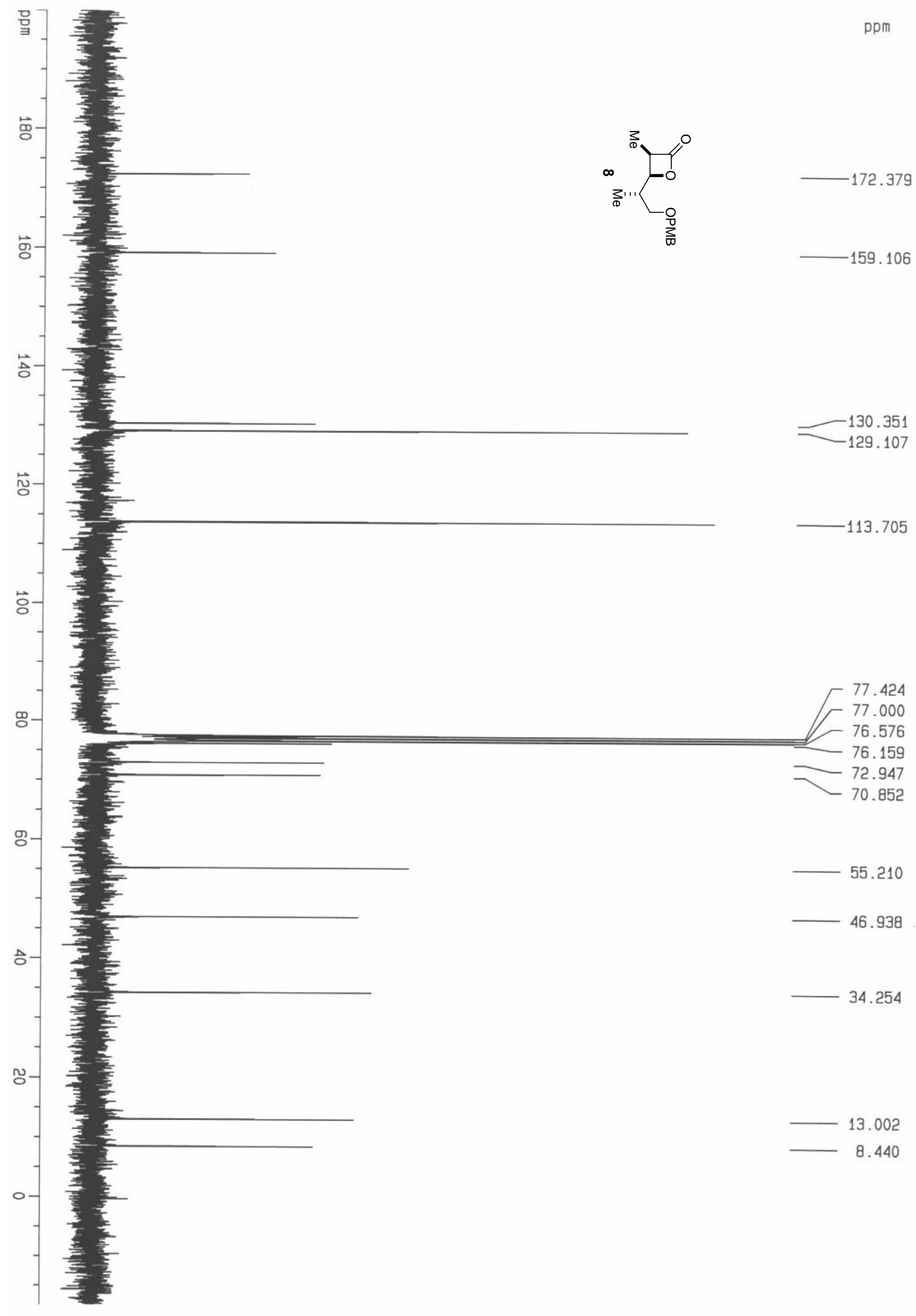




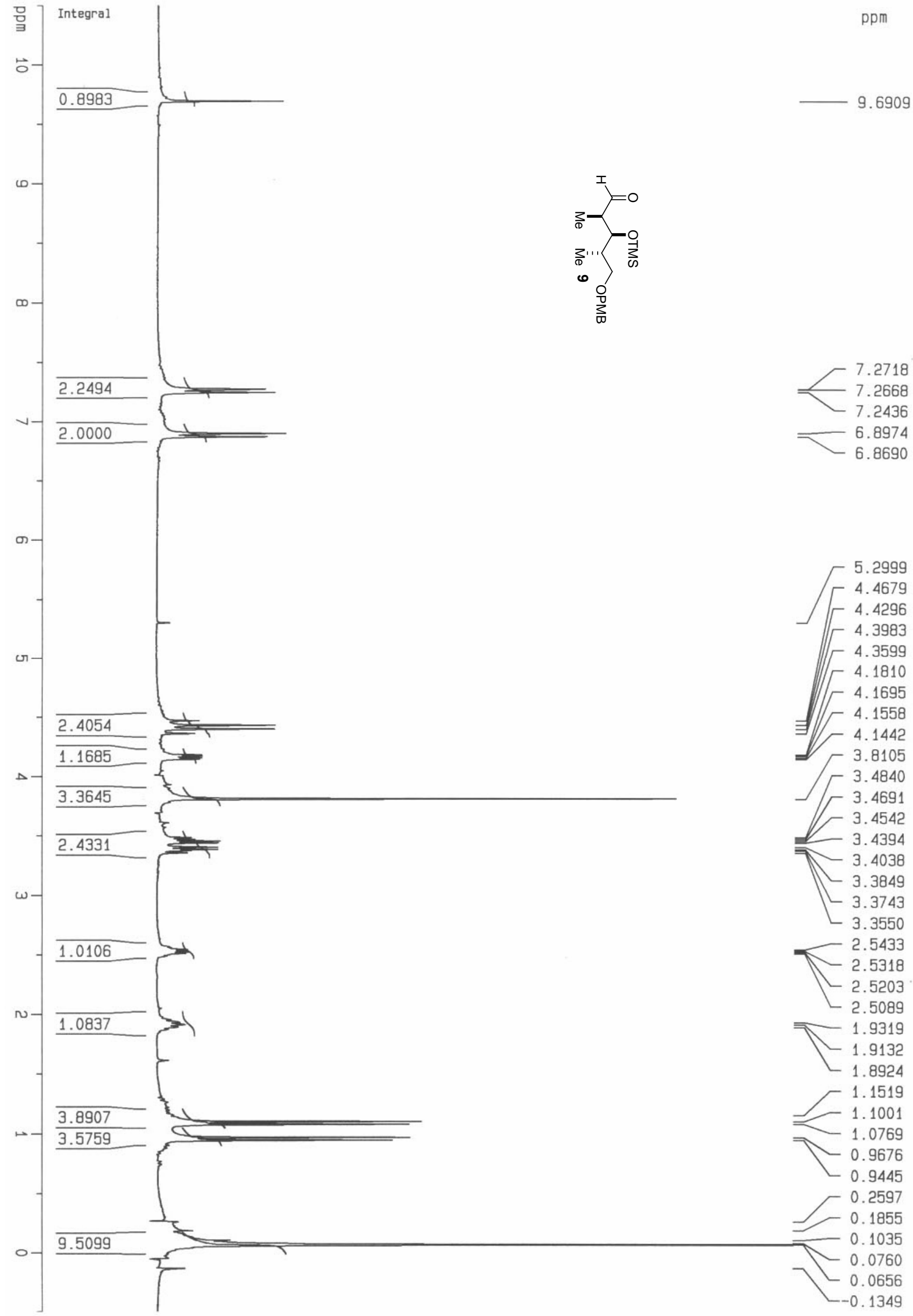




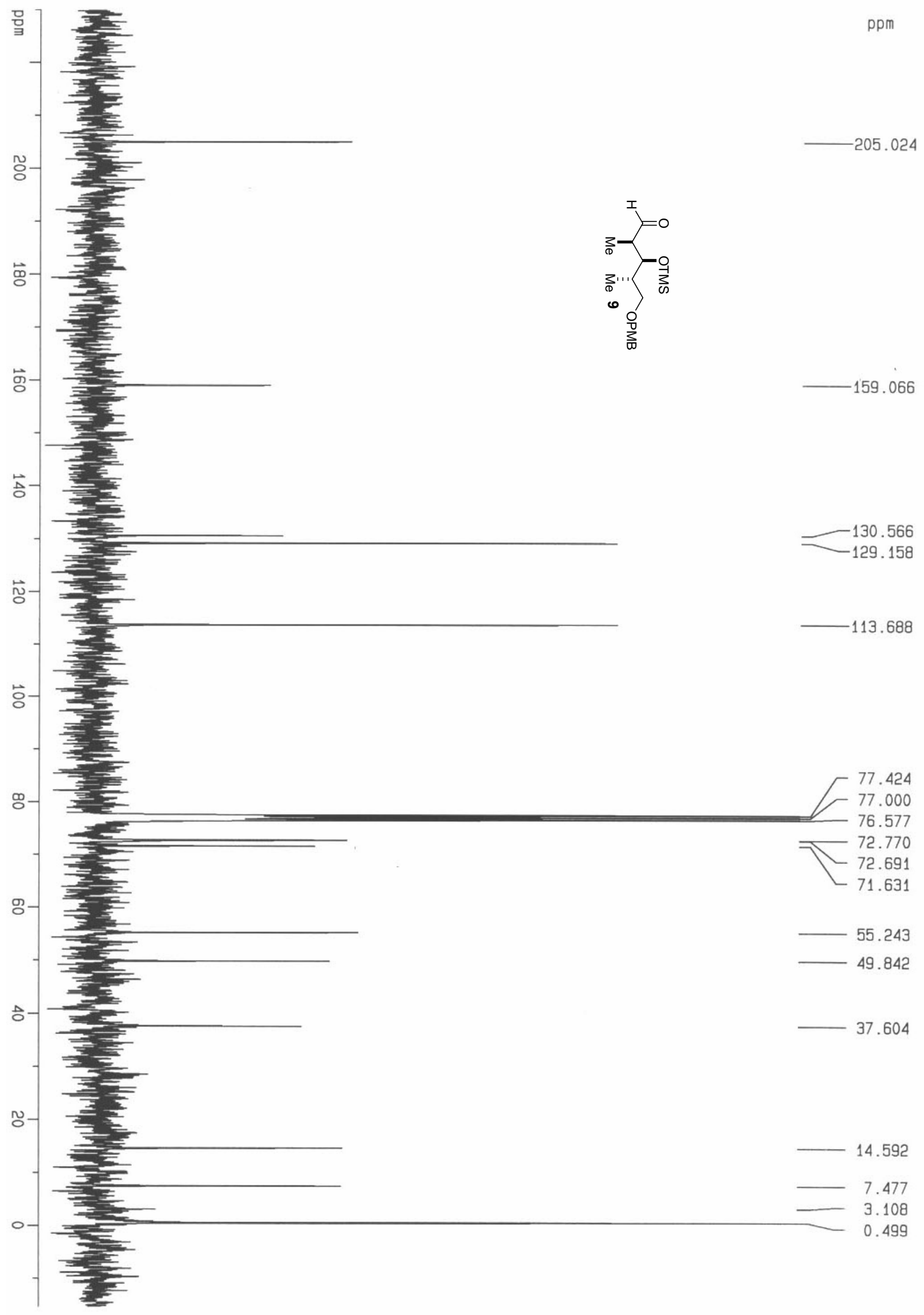




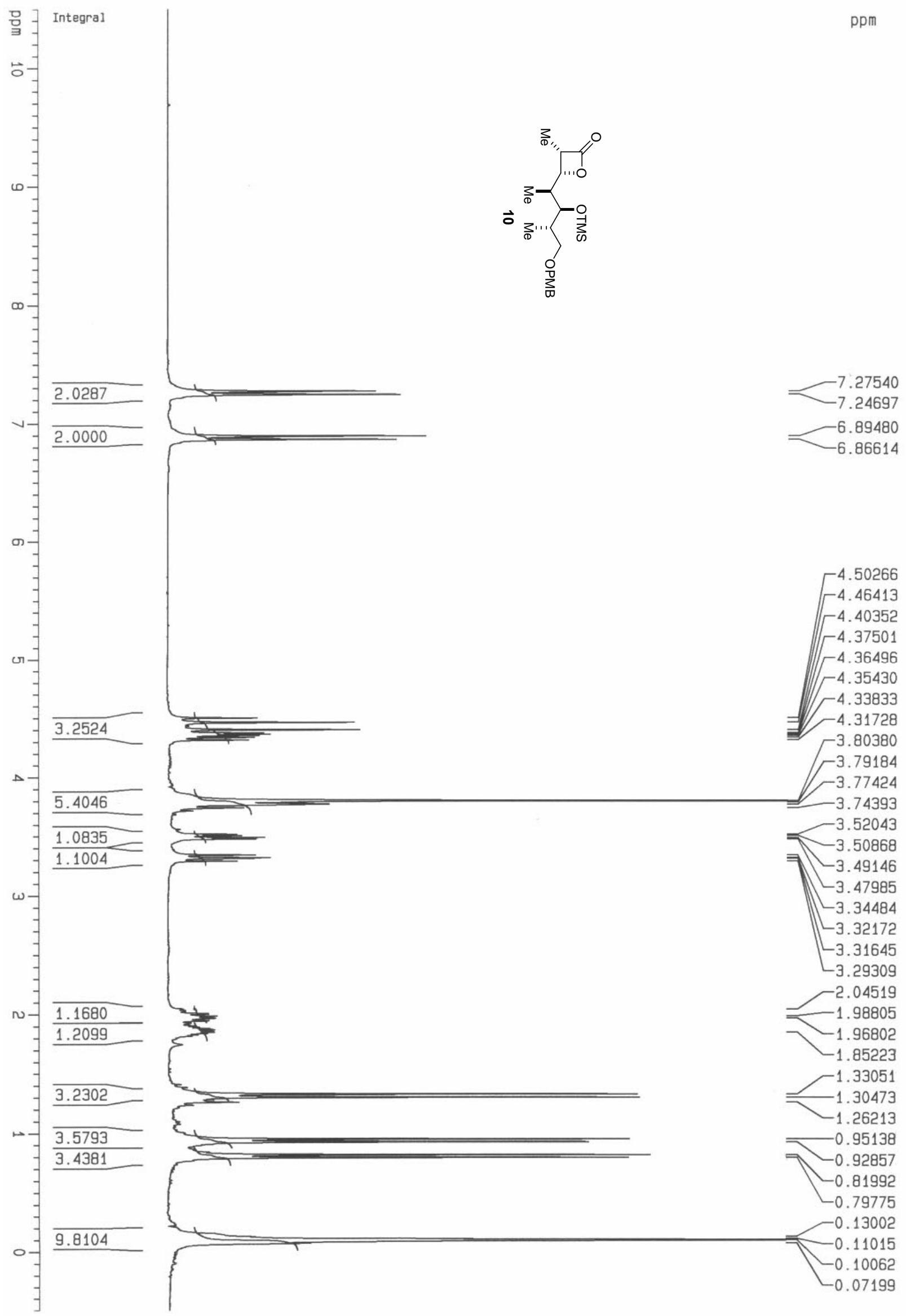




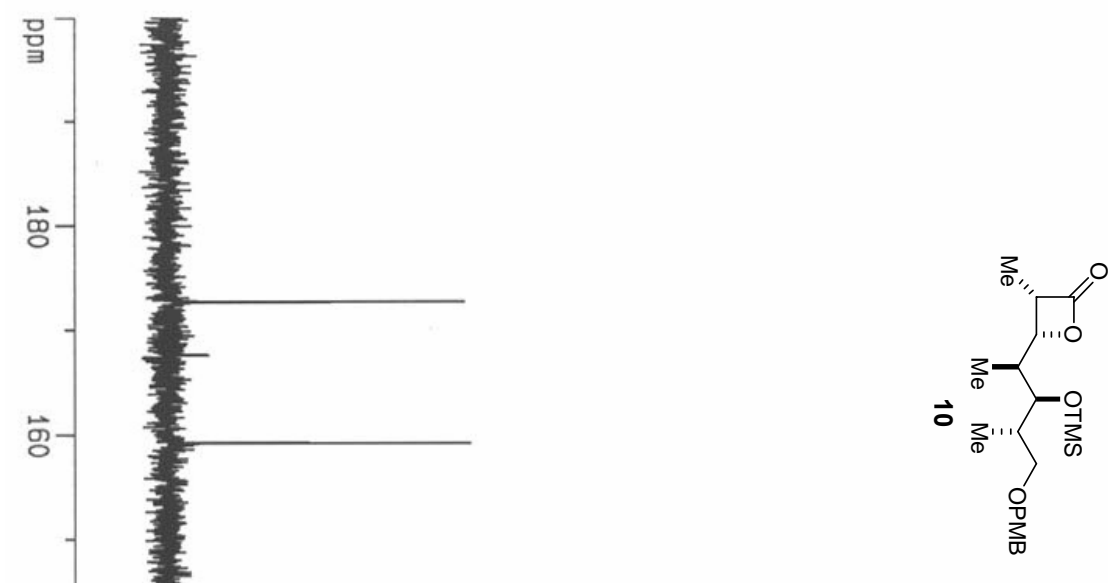

ppm

䓊

용
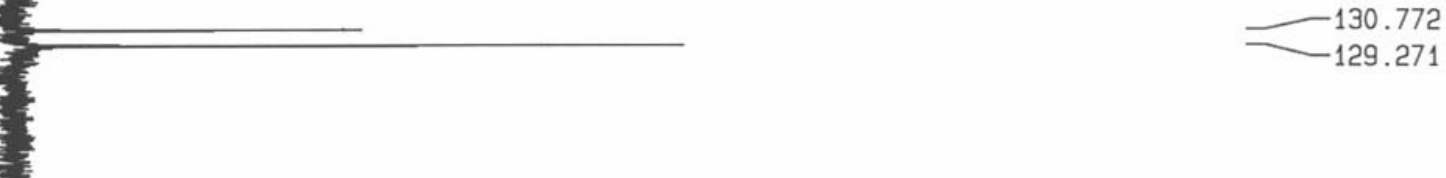

홍

ø

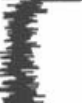

8-
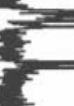

g-

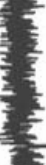

t.
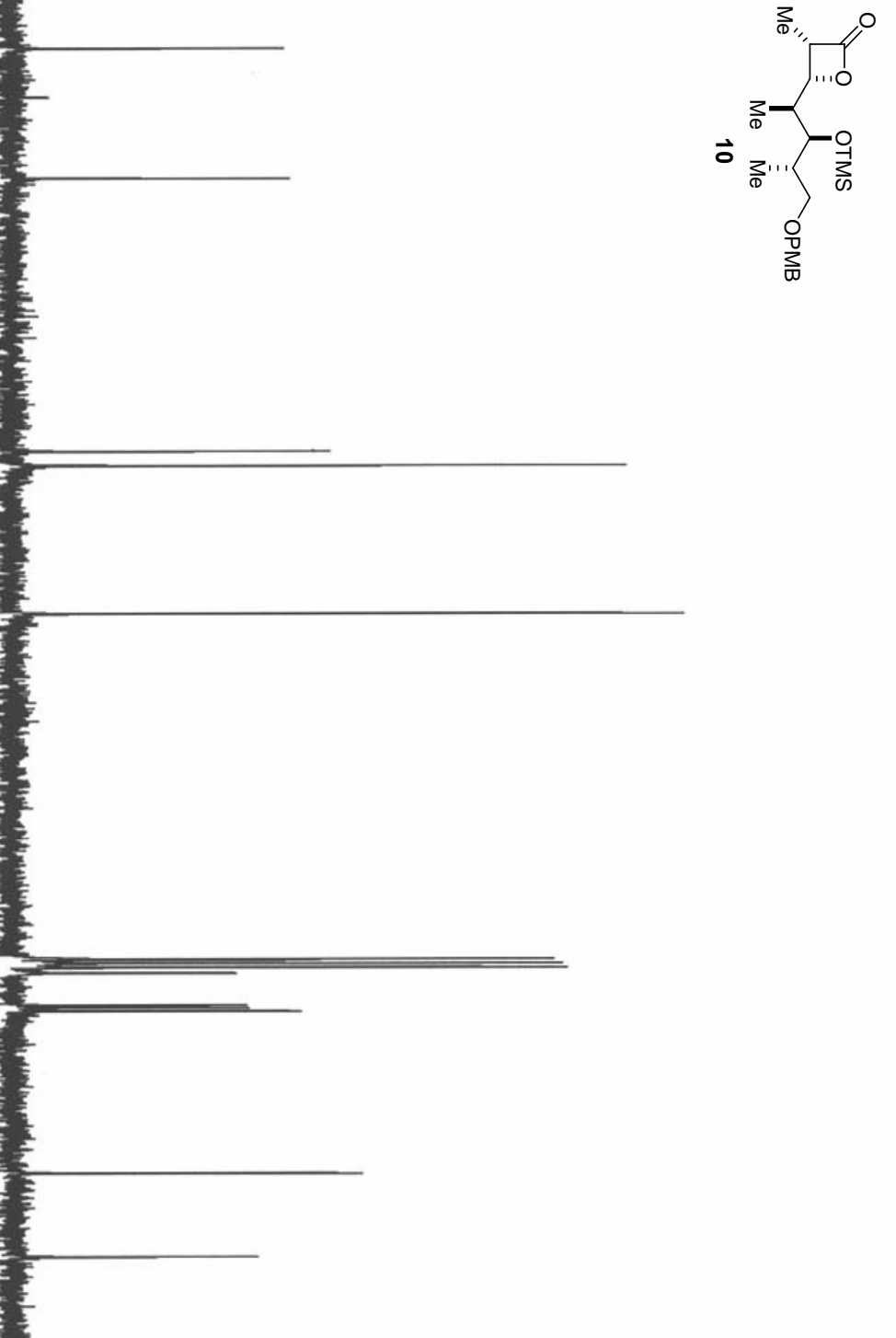

$-172.704$

$-159.189$

厂 77.642

77.218

$-76.794$

76.205

72.859

$-72.636$

$\leftarrow 72.249$

55.352

46.643

o

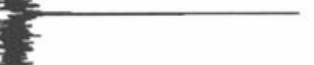

37.627

35.324

กำ

(2)

14.436

8.539

- 7.814

1.071

0.675

o-

0.279 


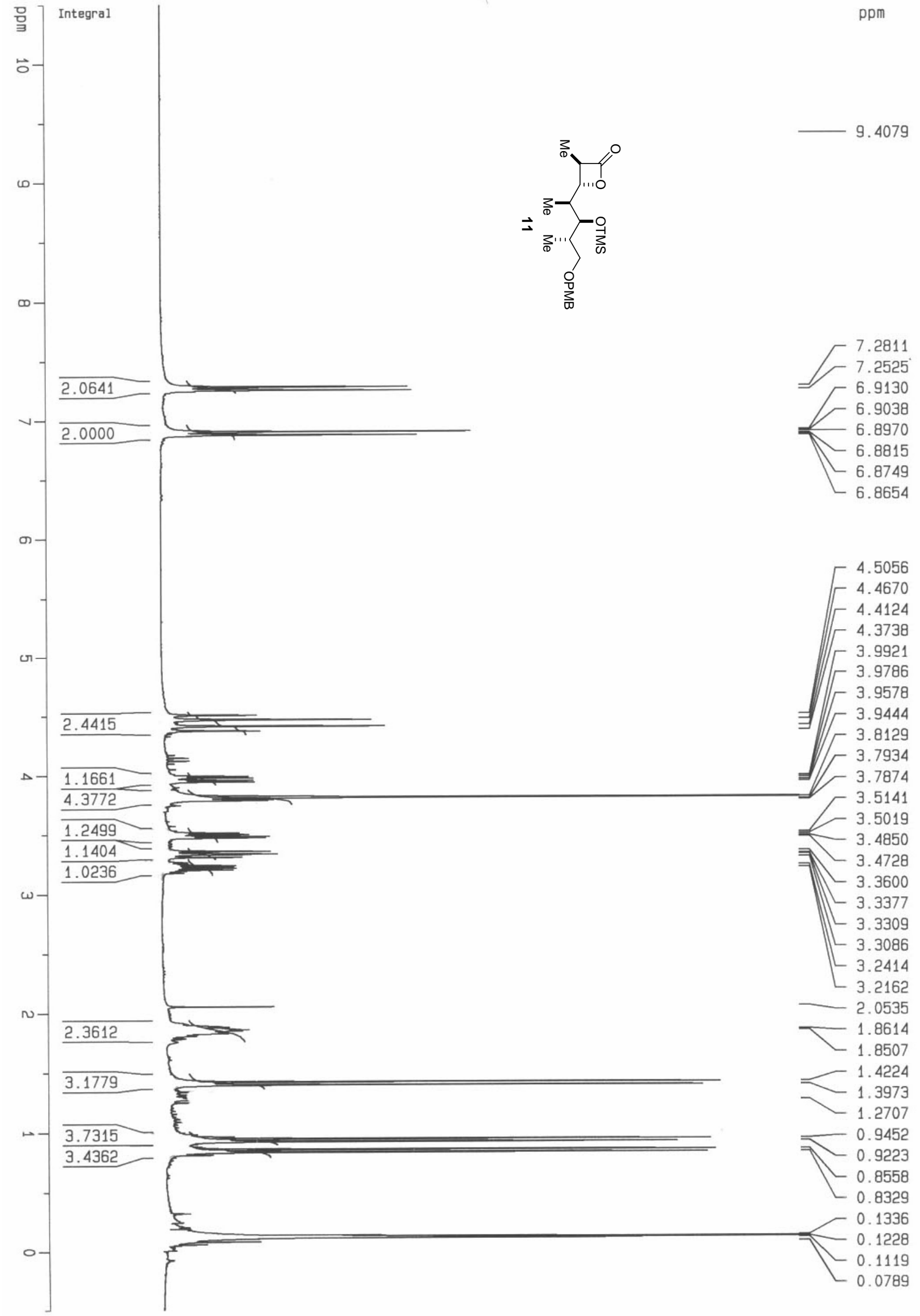



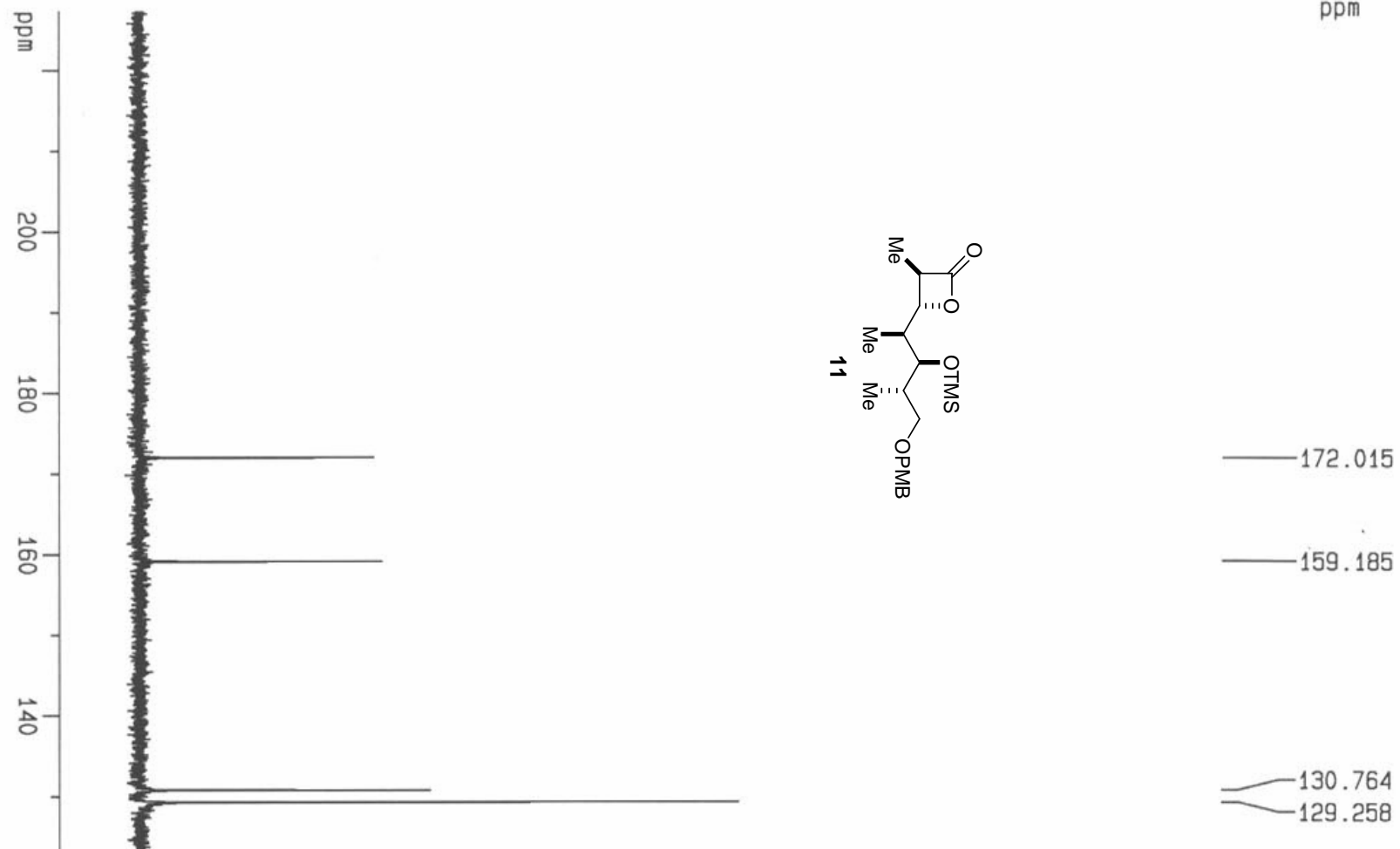

$\overrightarrow{8}$

$$
\text { จิ }
$$




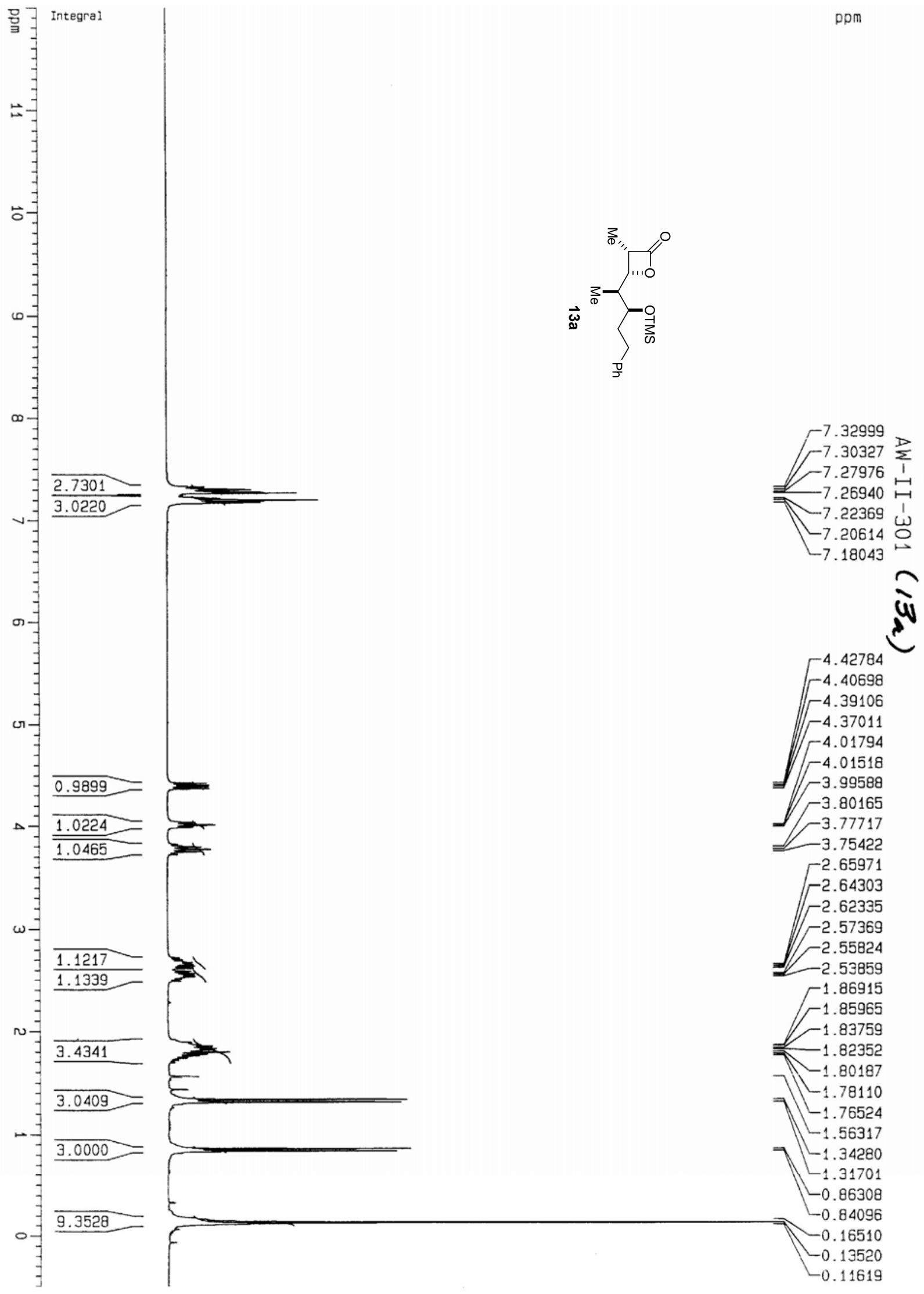



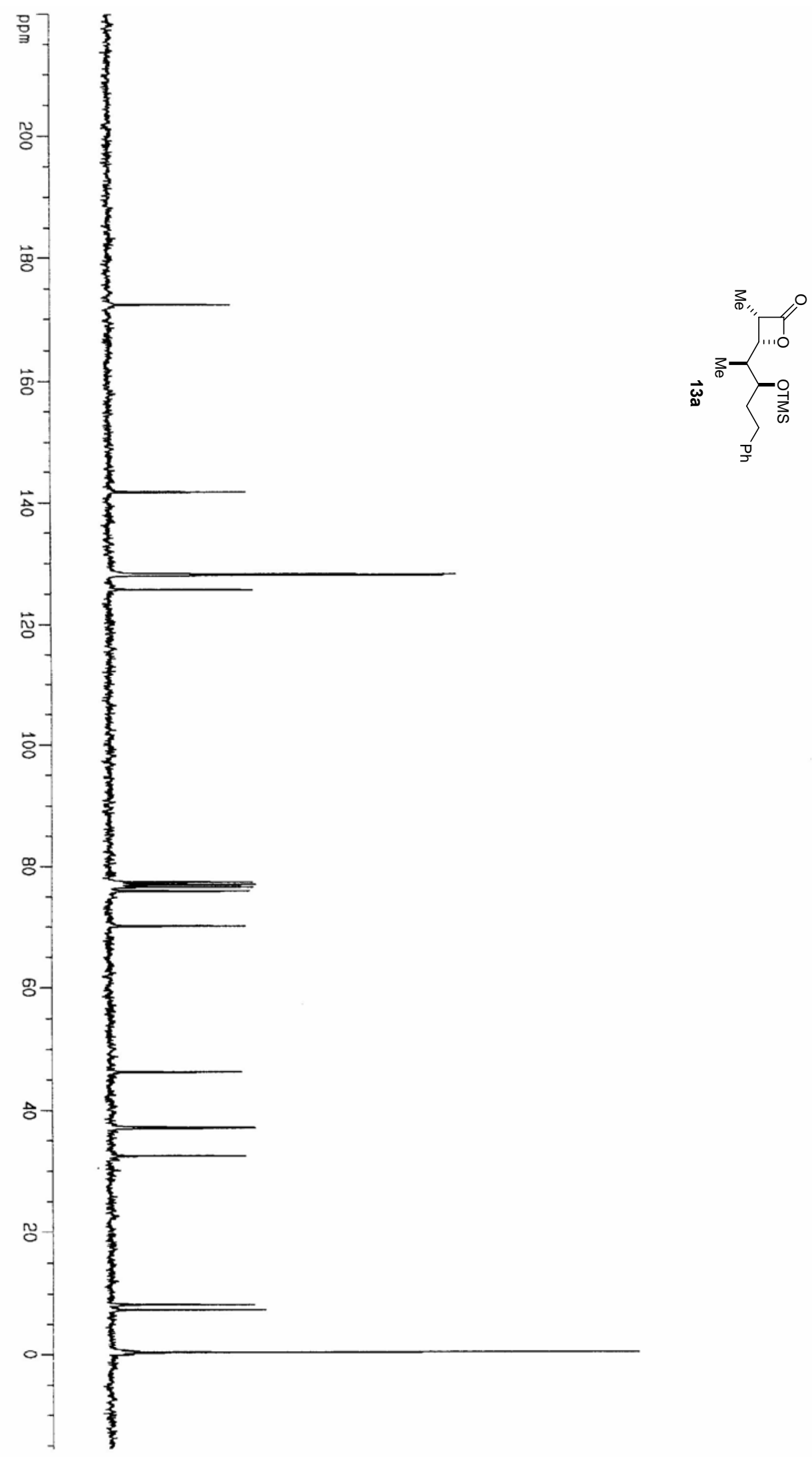

$\mathrm{ppm}$
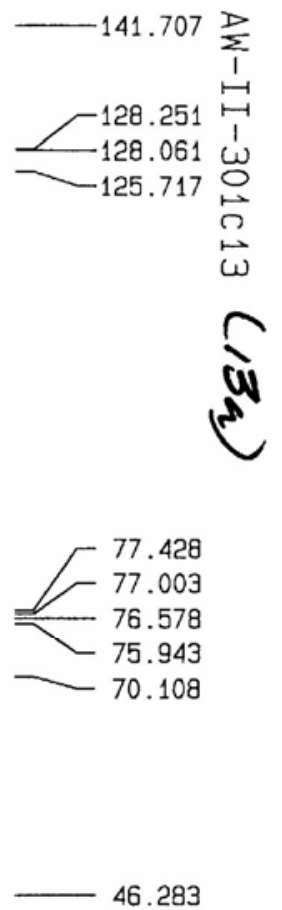

37.193

37.011

32.500

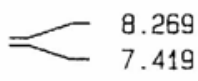

$\longrightarrow \quad 0.345$ 

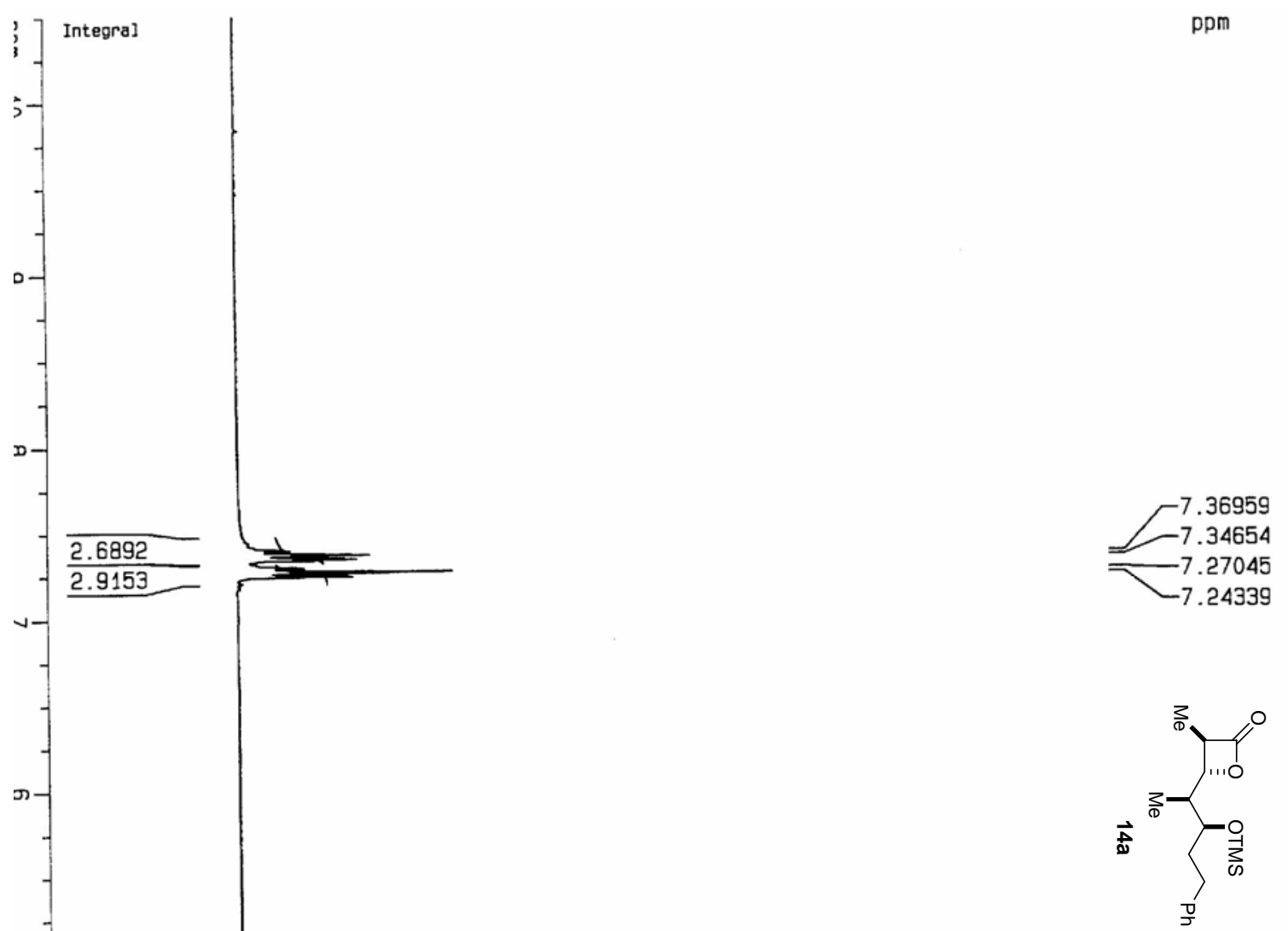

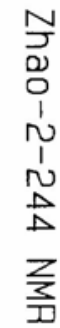
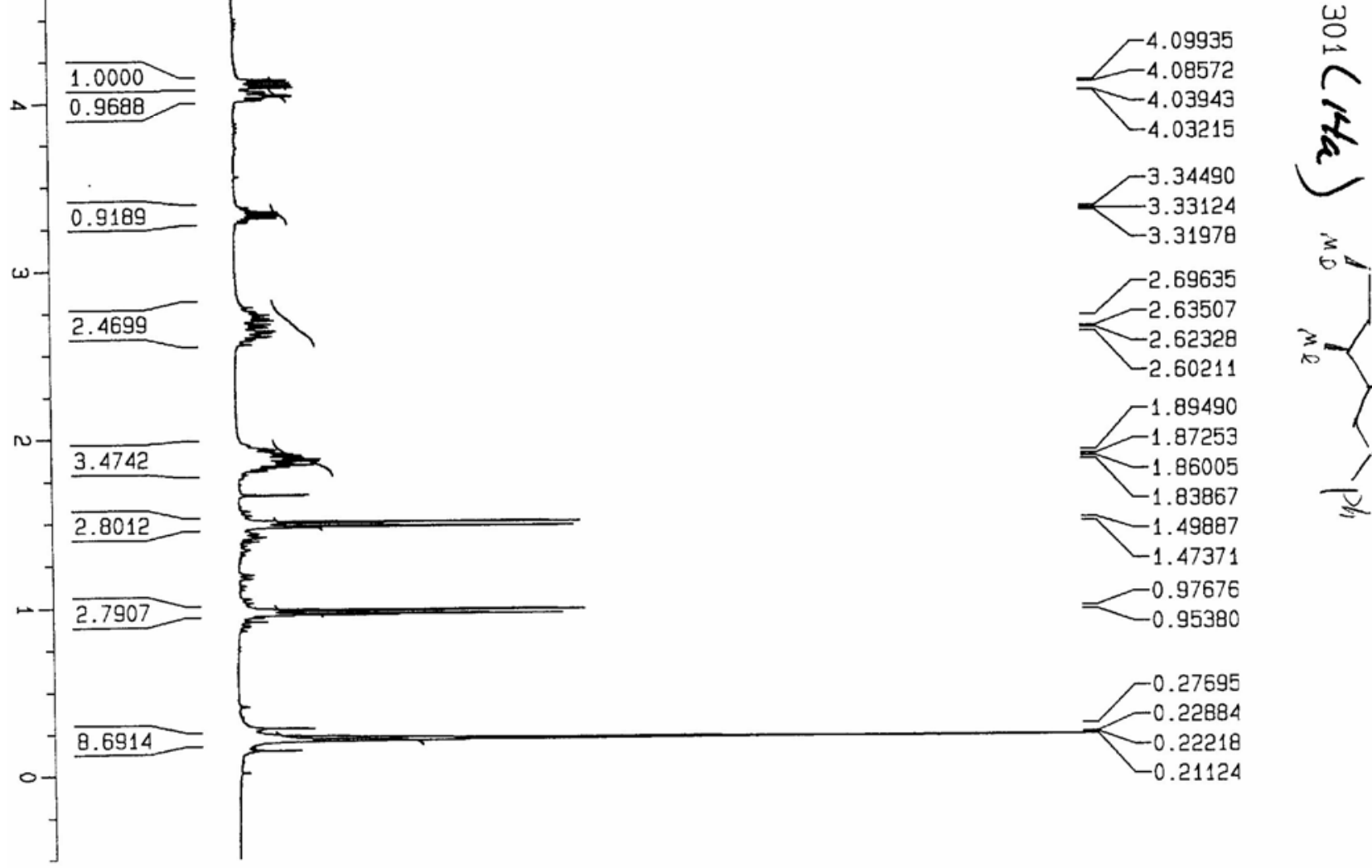

$-2.69635$

$-2.63507$

$-2.62328$

$-2.60211$

$-1.89490$

$-1.87253$

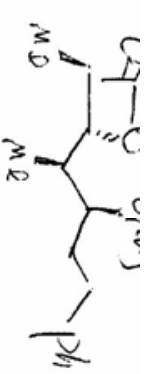

$-1.83867$

$\longrightarrow 1.49887$

$-1.47371$

$-0.97676$

$-0.95380$

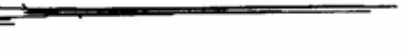

$-0.27695$

$-0.22884$

$-0.22218$

$-0.21124$ 


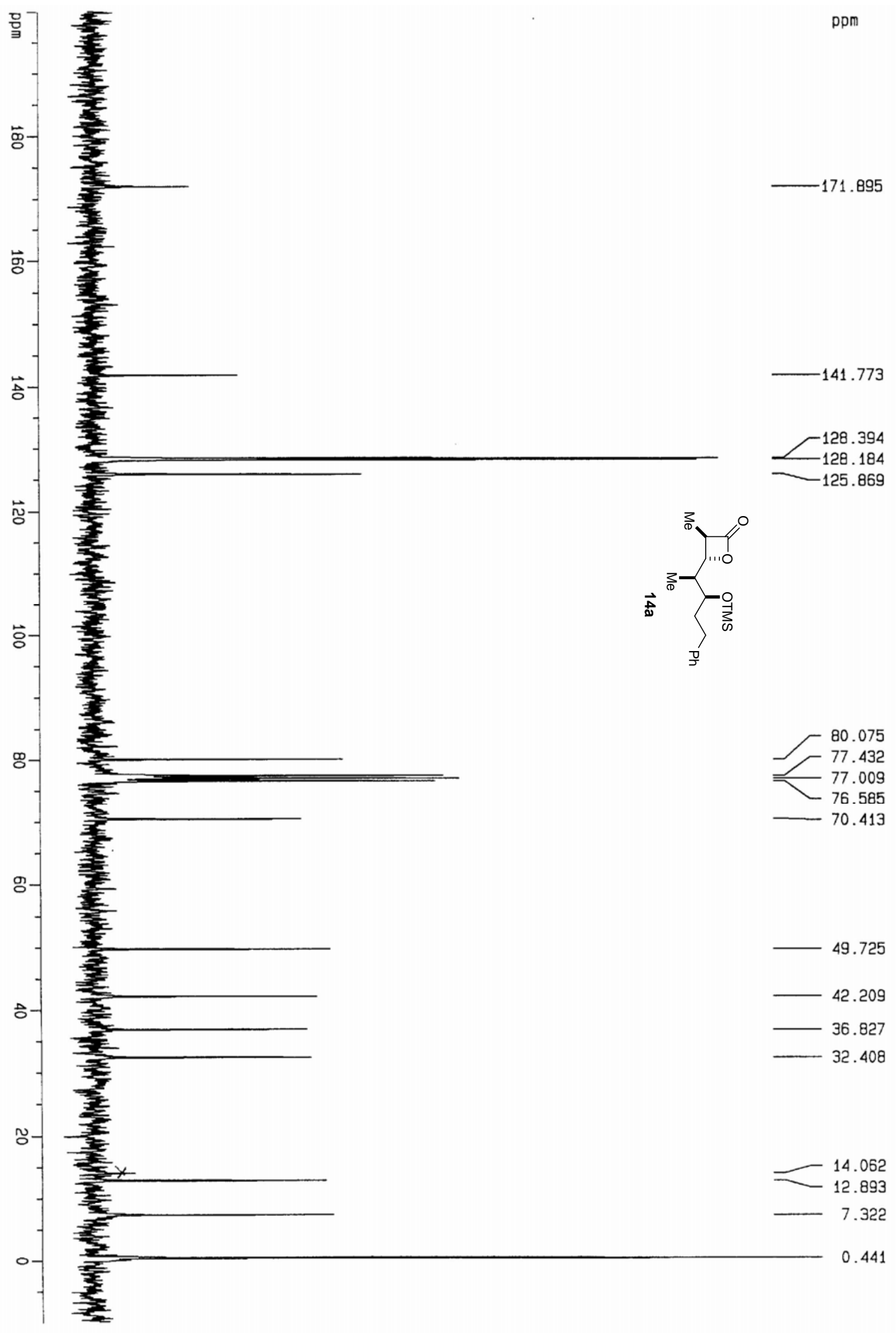




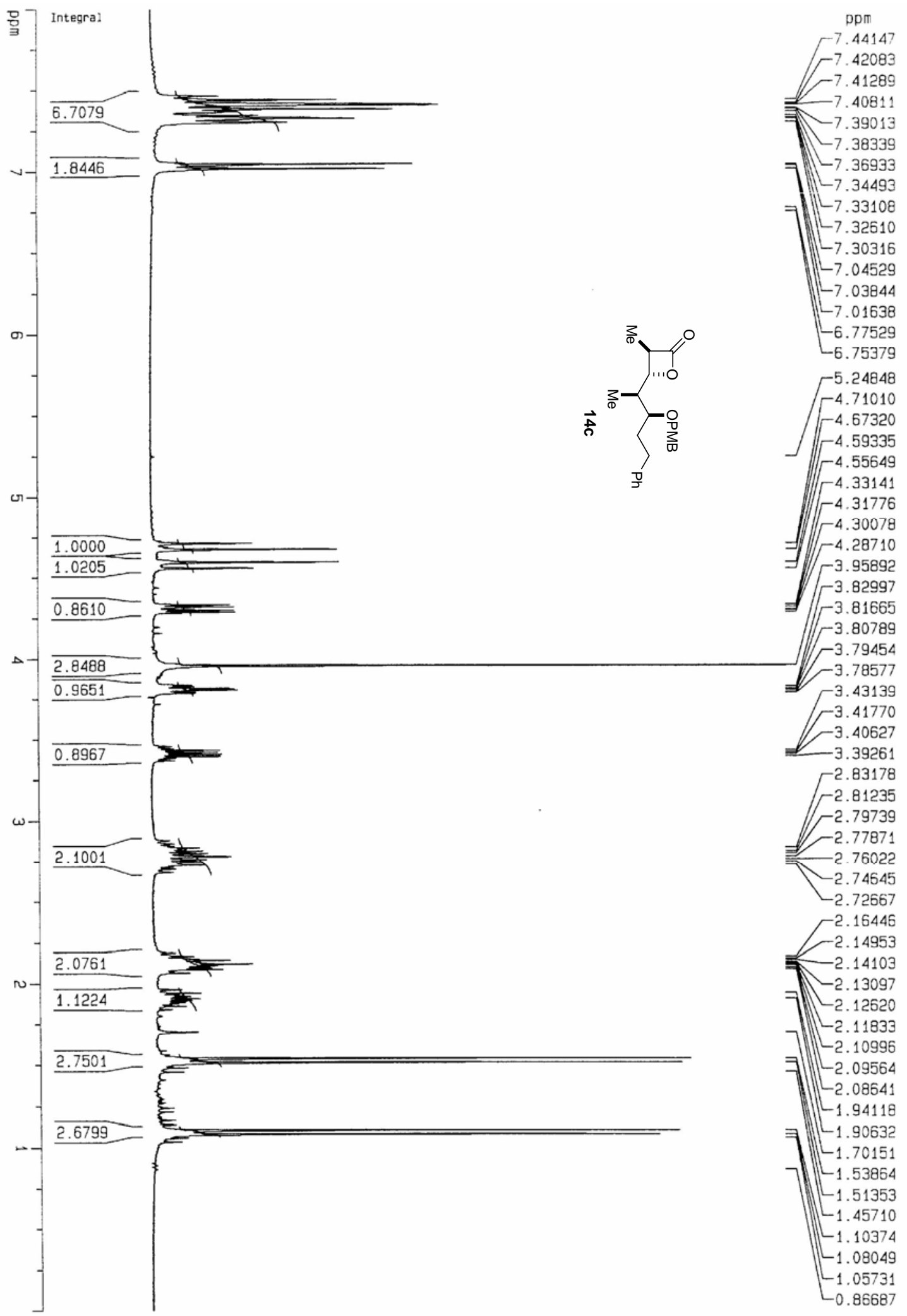



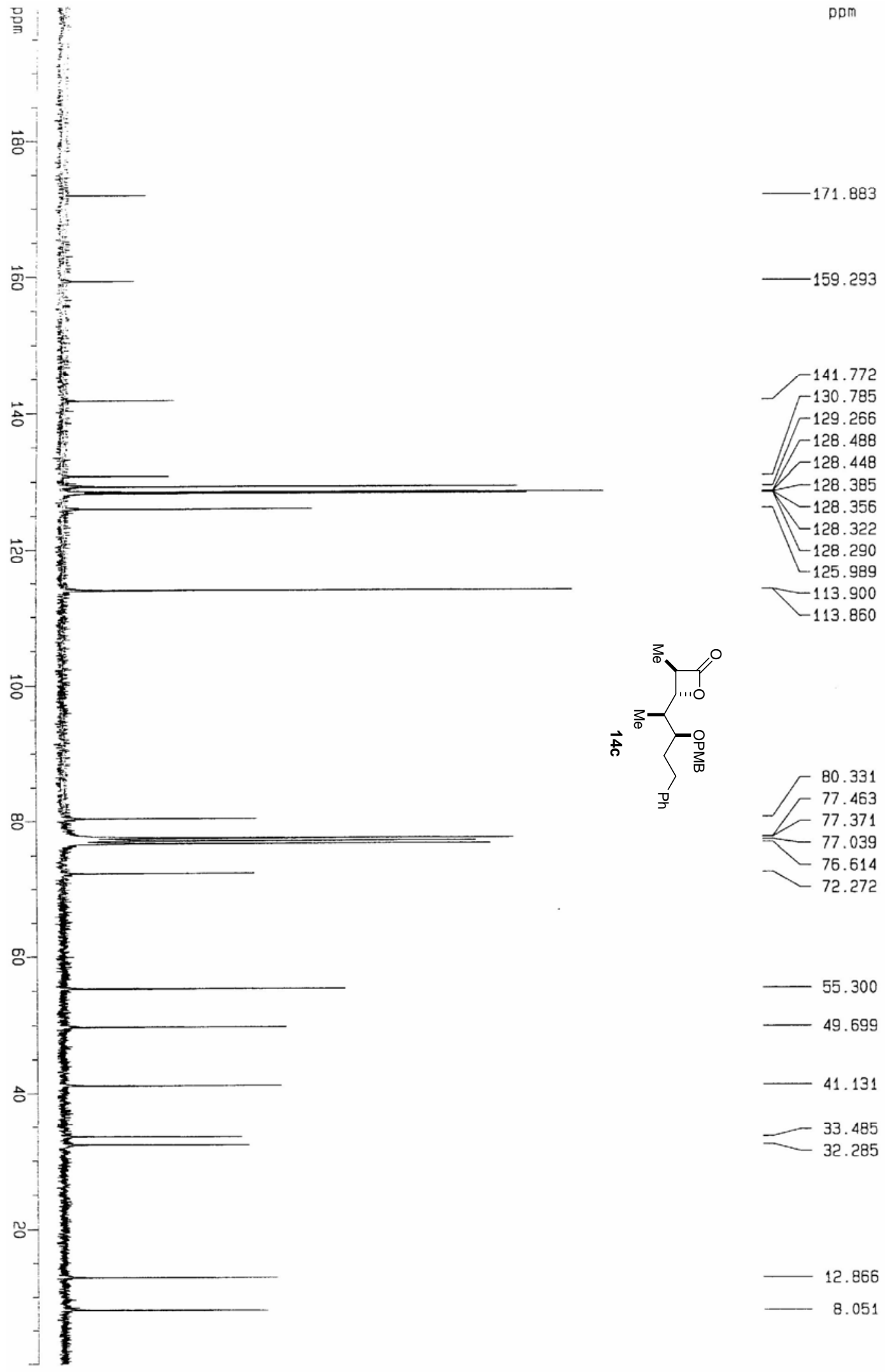<smiles>CCCC(OC)C(C)C1OC(=O)C(C)[C@@H]1C</smiles>

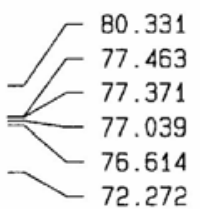

55.300

49.699

41.131

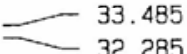

12.866

8.051 


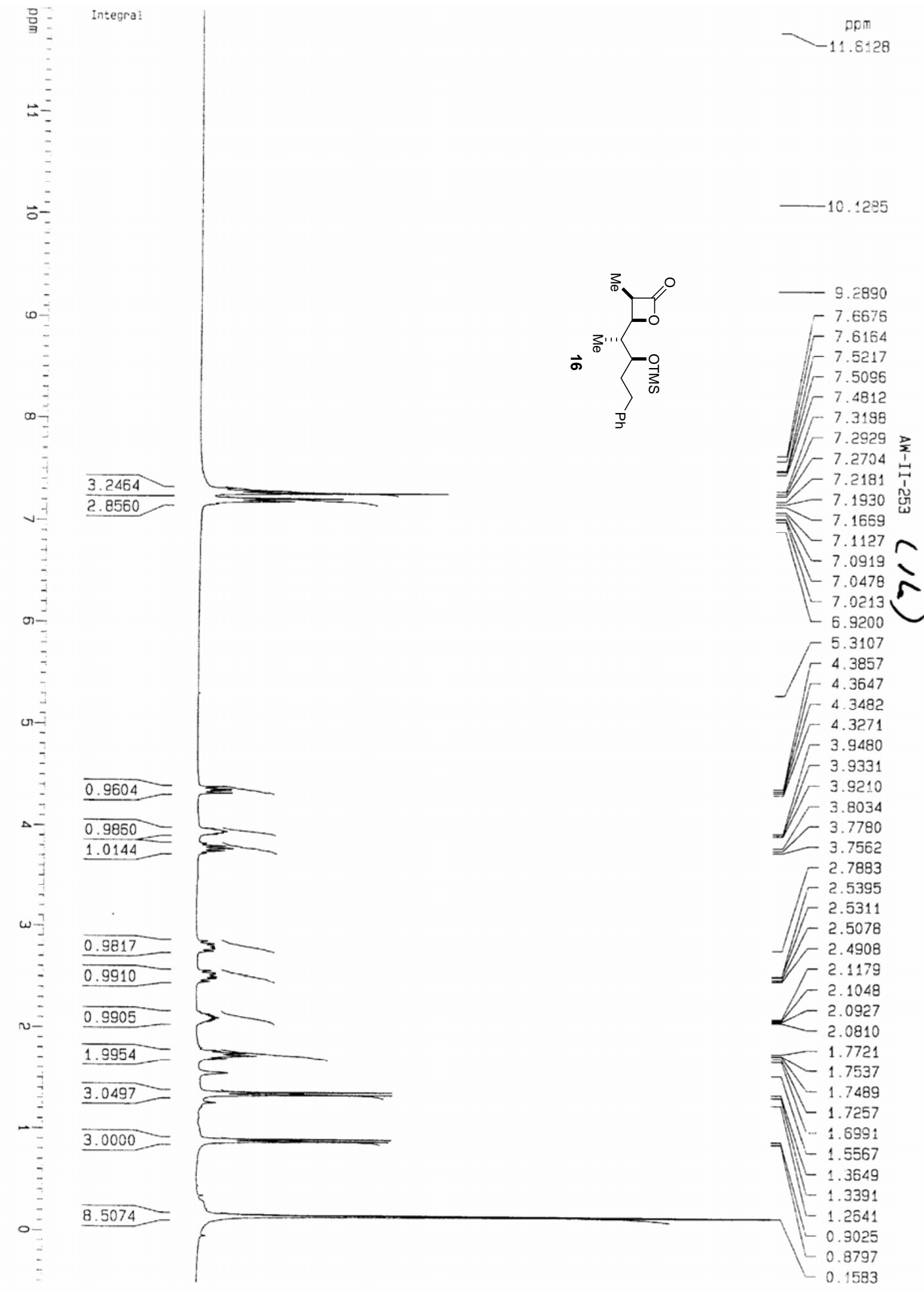




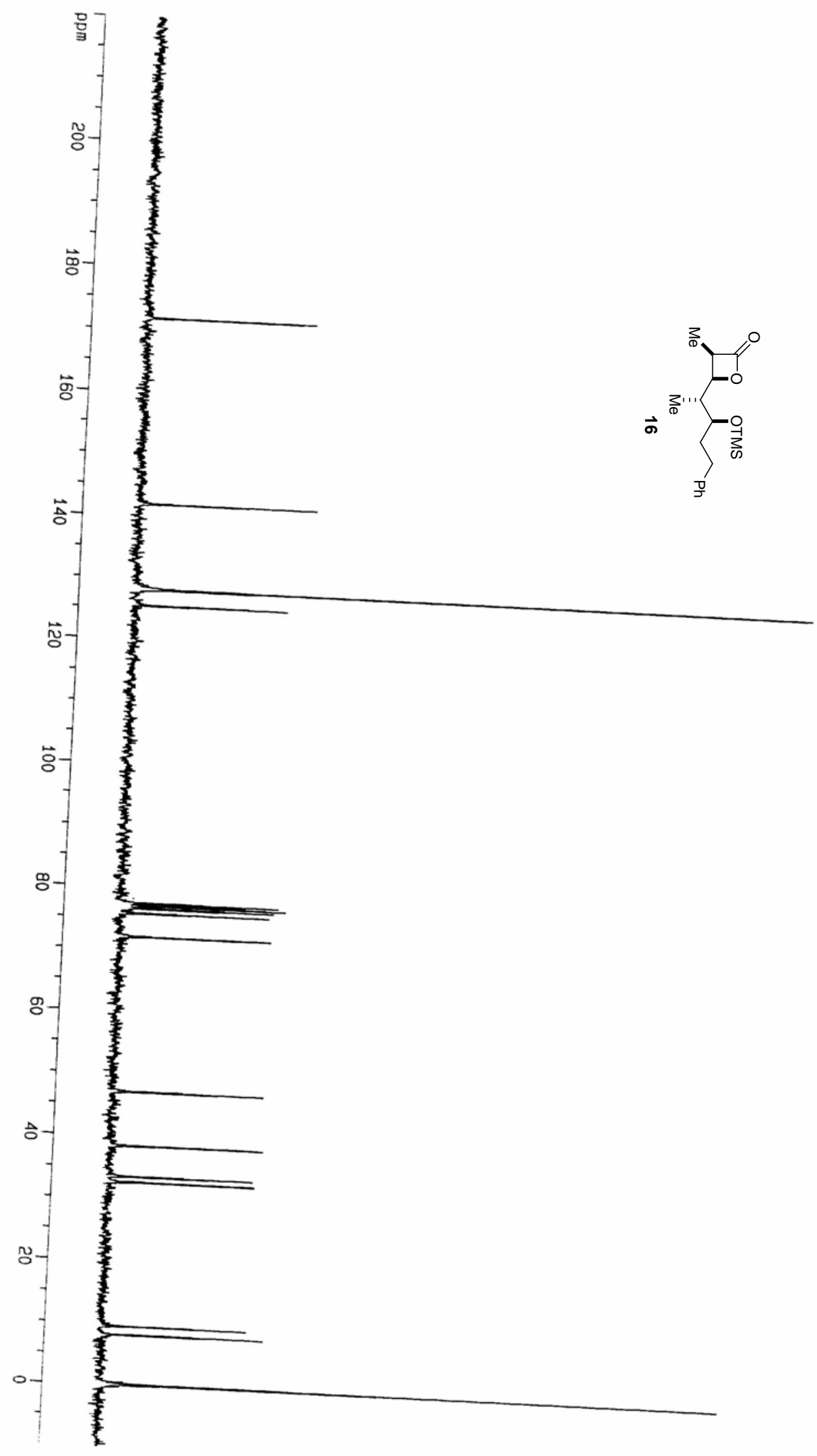

$\mathrm{ppm}$

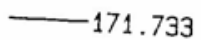

-141.906 $\frac{-1}{\frac{3}{\omega}}$

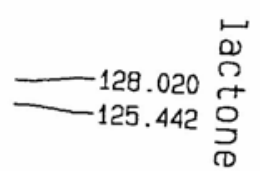

$\curvearrowright$

え

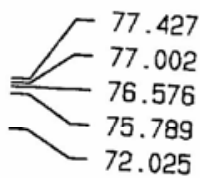

$-47.128$

$-38.506$

$-33.493$

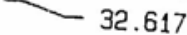

$\simeq \quad 9.536$

8.189

0.092 


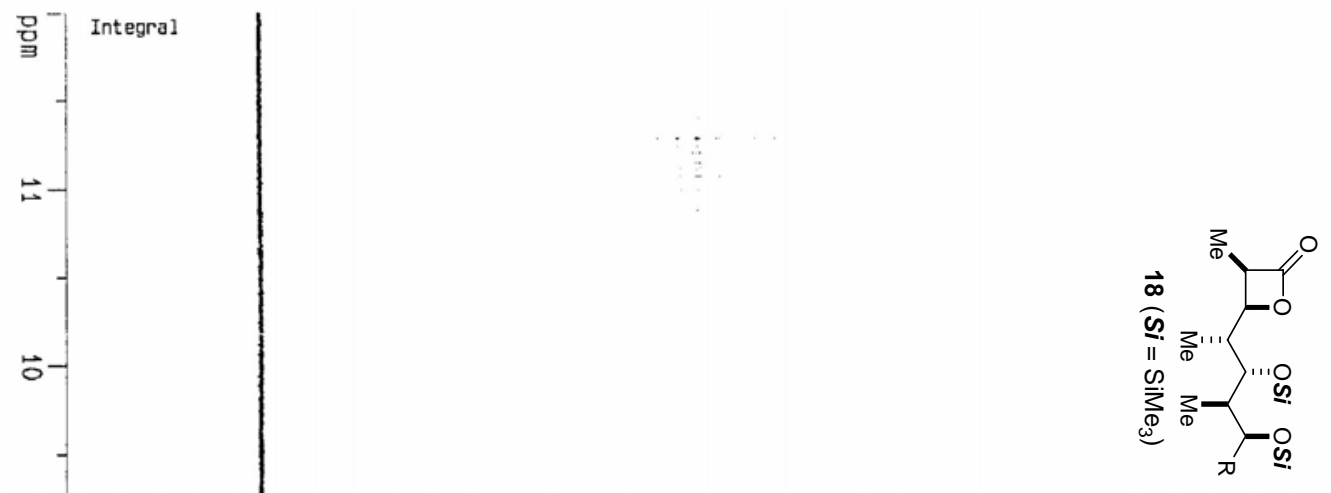

ppm

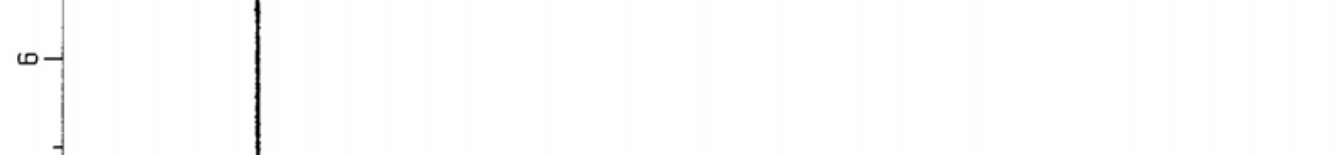

$\infty$

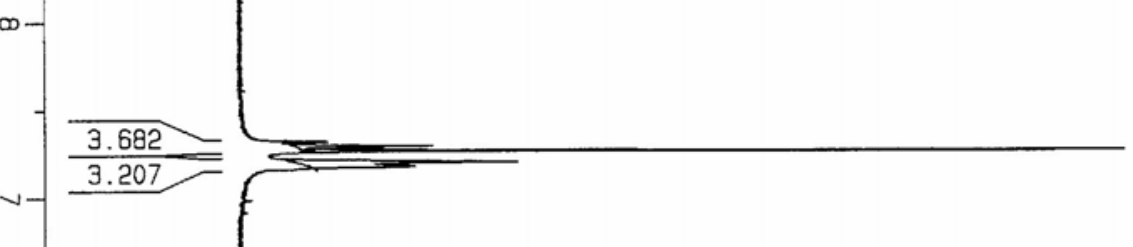$$
\Delta
$$$$
\triangle
$$$$
\frac{1.000}{2.056}
$$

$\omega-$

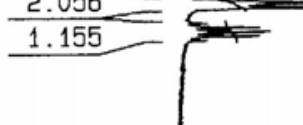

$$
\text { N- }
$$
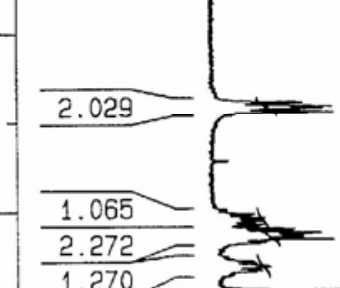

$-\frac{1.270}{\frac{3.112}{2.878}}$

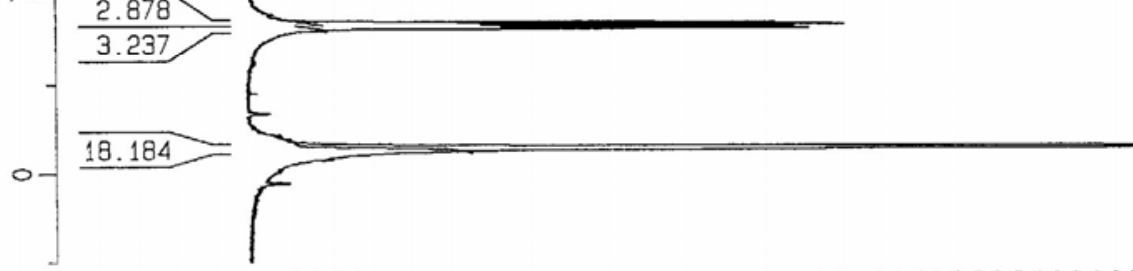

7.6149

F 7.3231

- 7.3173

$-7.2982$

$-7.2690$

7.2079

$7-7.1984$

$-7.1910$

$-7.1822$

- 6.9899

- 6.9180

$-5.0209$

$\Gamma^{4} .3961$

$-4.3752$

ז 4.3591

- 4.3381

3.9391

F. 3.9345

1 3.9222

- 3.9116

- 3.7739

- 3.7693

2.6146

2.5932

- 2.5808

- 2.5594

1.9025

$=1.8829$

F 1.8779

- 1.8676

$-1.8606$

$-1.8453$

1.5549

$-1.4410$

$-1.3482$

$\llcorner 1.3223$

$7-0.8447$

$-0.8329$

$-0.8214$

L 0.8108

$-0.1412$

0.1382

0.0748 


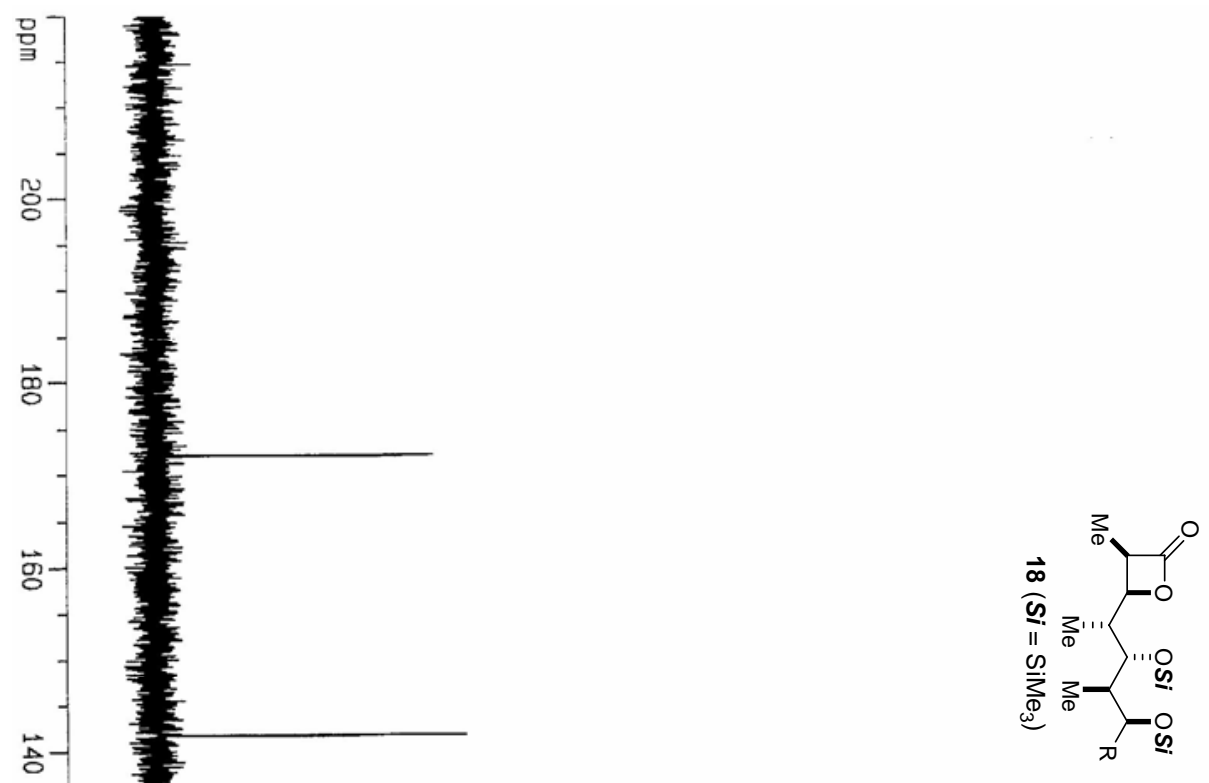

$\mathrm{ppm}$
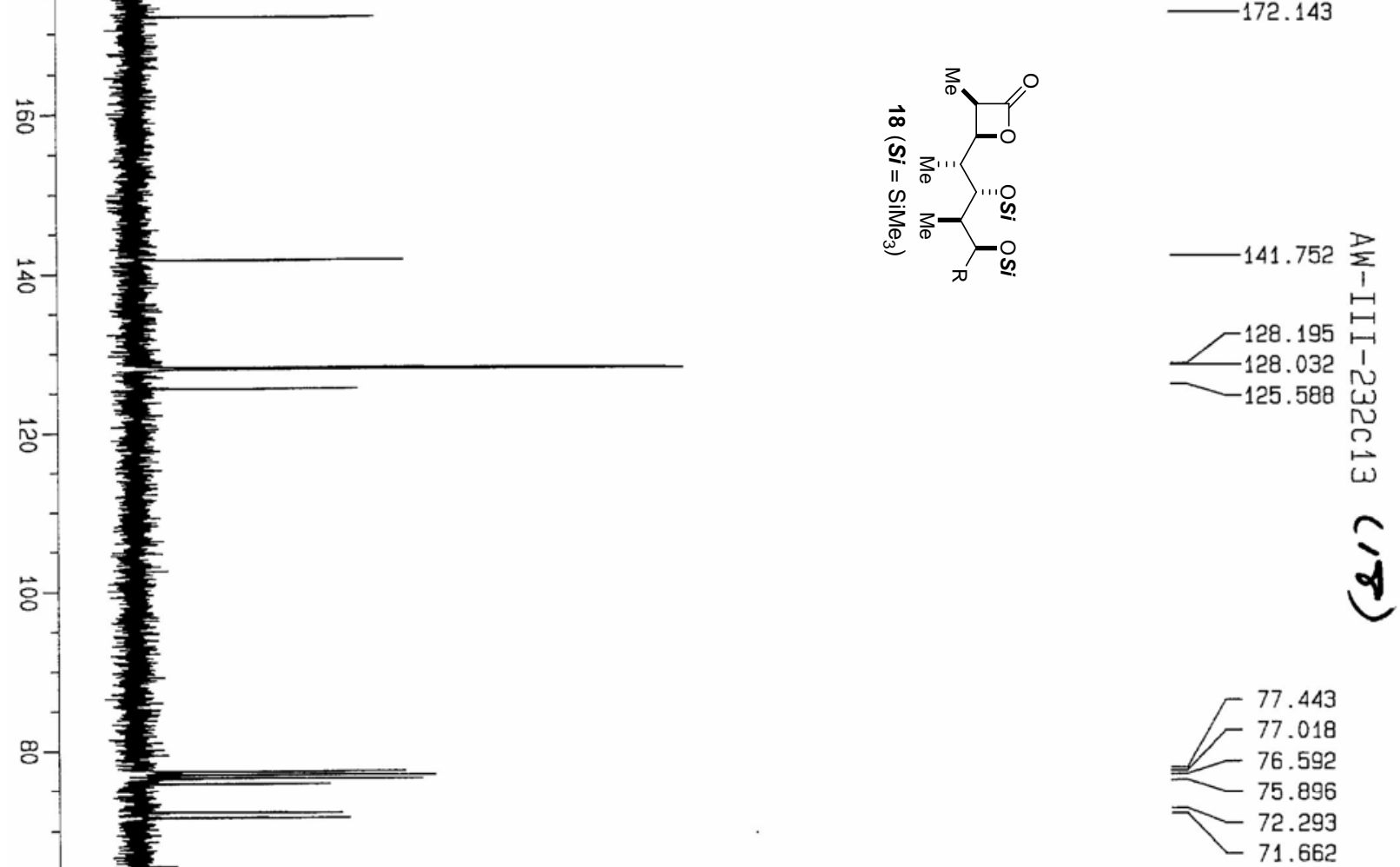

46.262

$-41.171$

$-37.291$

$-35.181$

$-32.023$

N

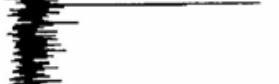

난

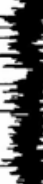

o-

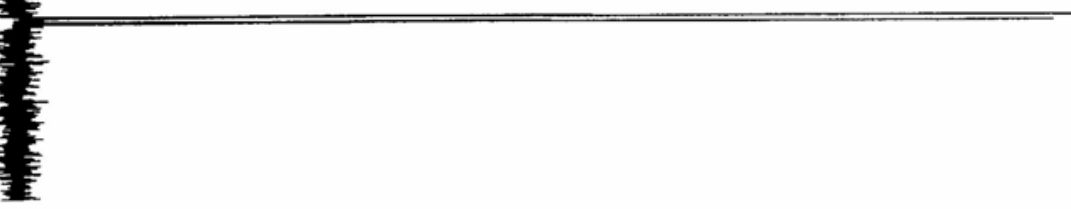

15.085

9.588

8.221

7.886

1.169

0.597 

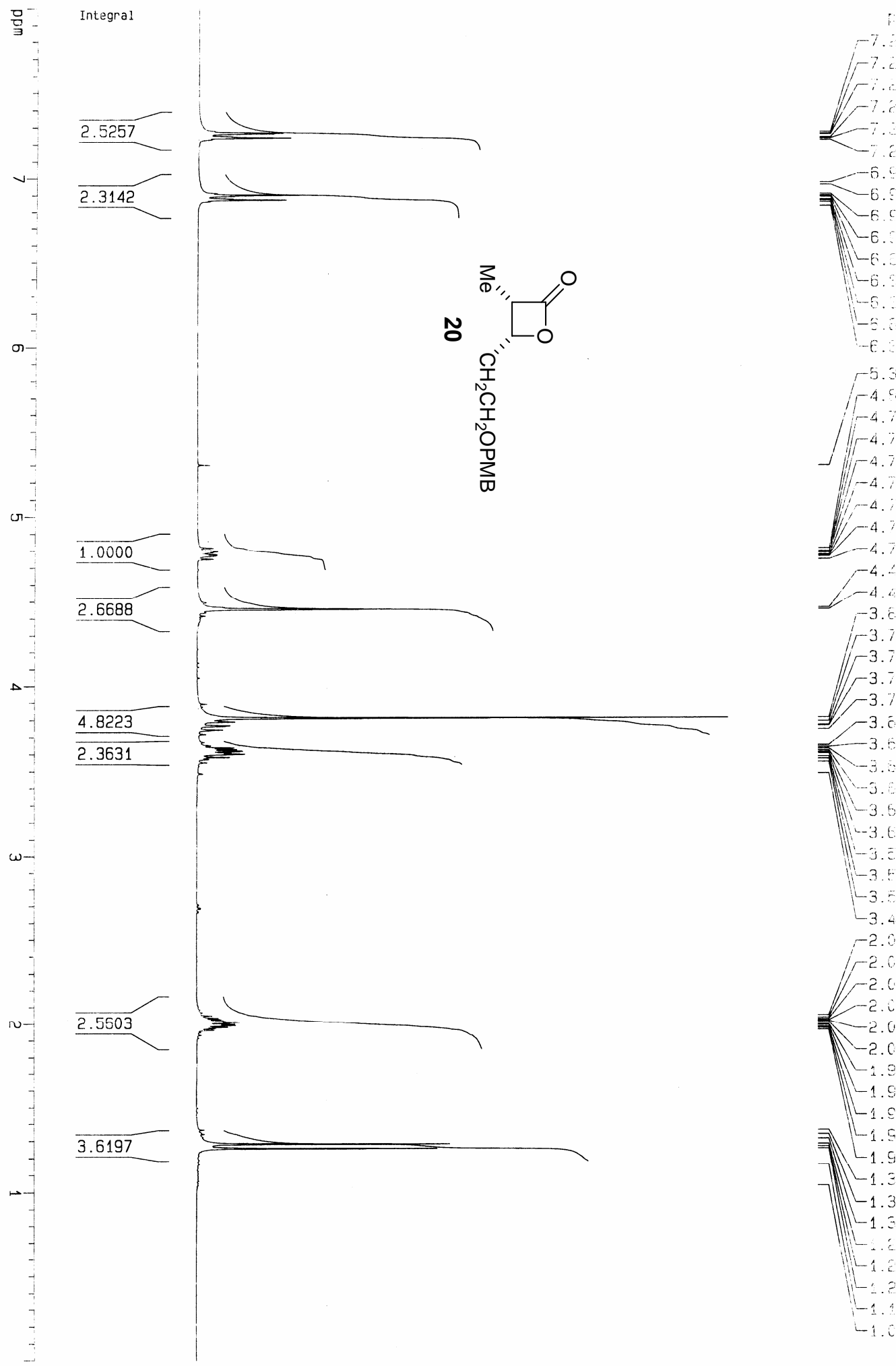

$--6$.

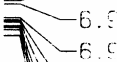

iil G $_{6}$

IILE.

146.

$-6$

$-E$.

$-5.5 \cdot 3$.

$-4.5$

$\iint^{-4}, \frac{\infty}{x}$

$\Gamma_{-4}^{4}=\frac{0}{\sim}$

$\| 11-4 ;+3$

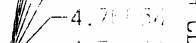

$-4 \ldots-\omega$

-4 .

$-3.6$

$-3.7$

$-3.7$

$-3.76$

$-3.7=2$

$\equiv-3.0$

$-3.6$

$\mathbb{- 3}$

If -3.6

$-3.6$.

$-3.2$

$-3.5-3$

ᄂ3.

$\bigsqcup_{3.4 \%} \cdot 3$

$-2.0695$

$/ / 2.0 .50$

-2.c-3

-2.6.

$-2.0553$

$-1, a$

$-1.5 \%$

L. 0 (a)

司 4.5

$-L_{1}$

$L_{1.3634}$

$-1.3 \ldots 9$

$-1.3 .89$

L- $1.6 \%$

Li.e.s

IL.

$-1.100$

Li.c. 01

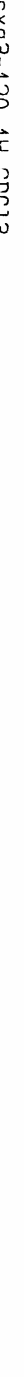




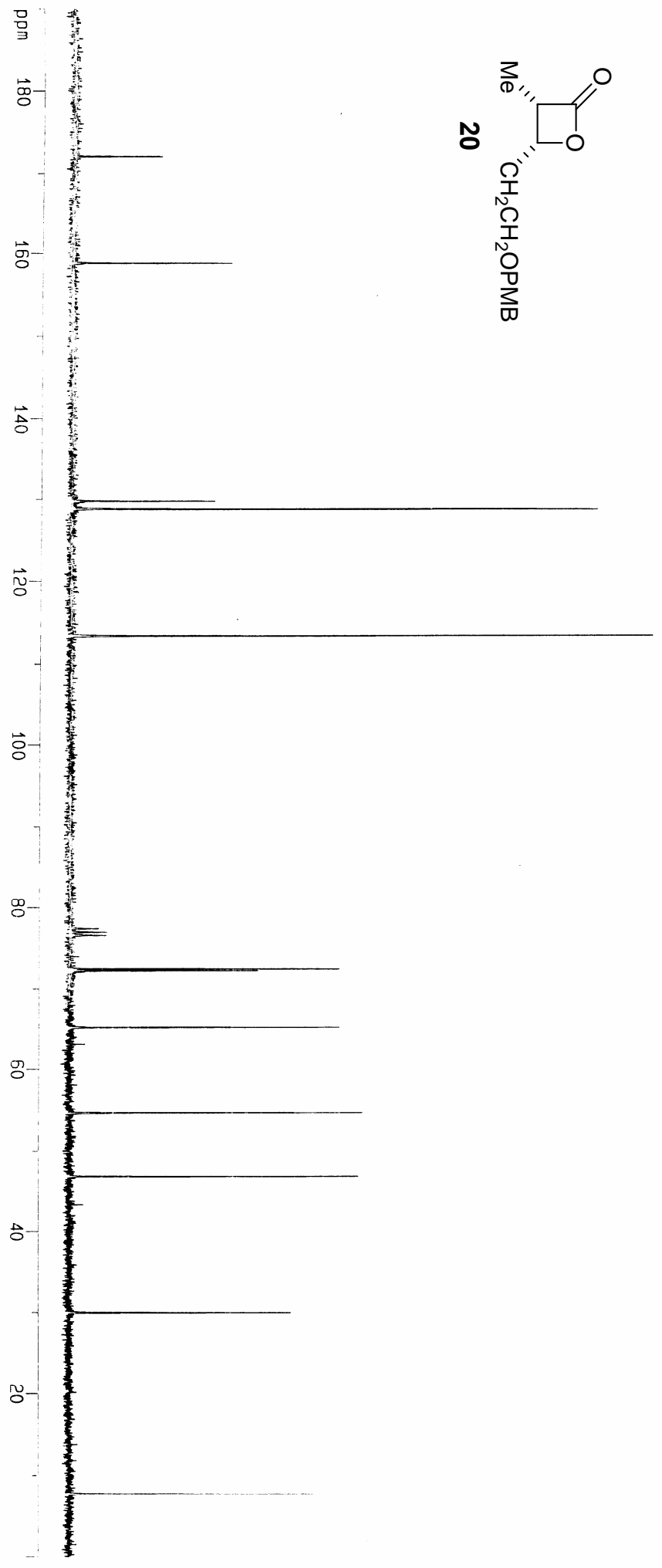

ppi
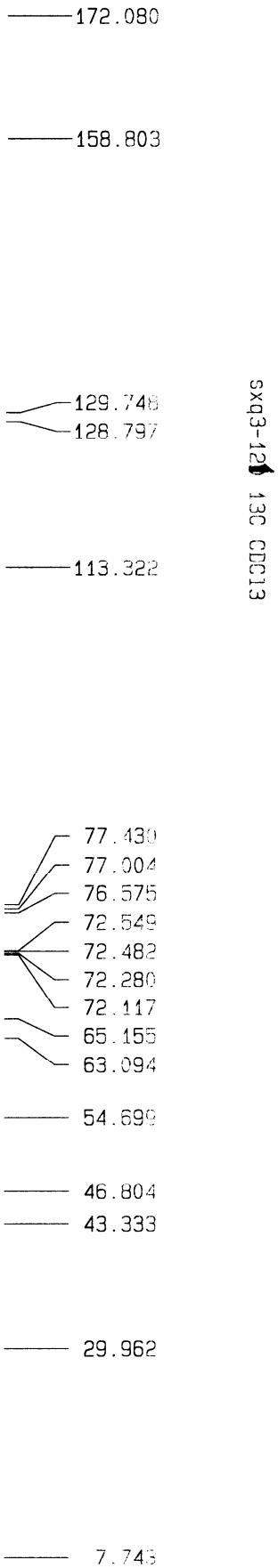

7.743 

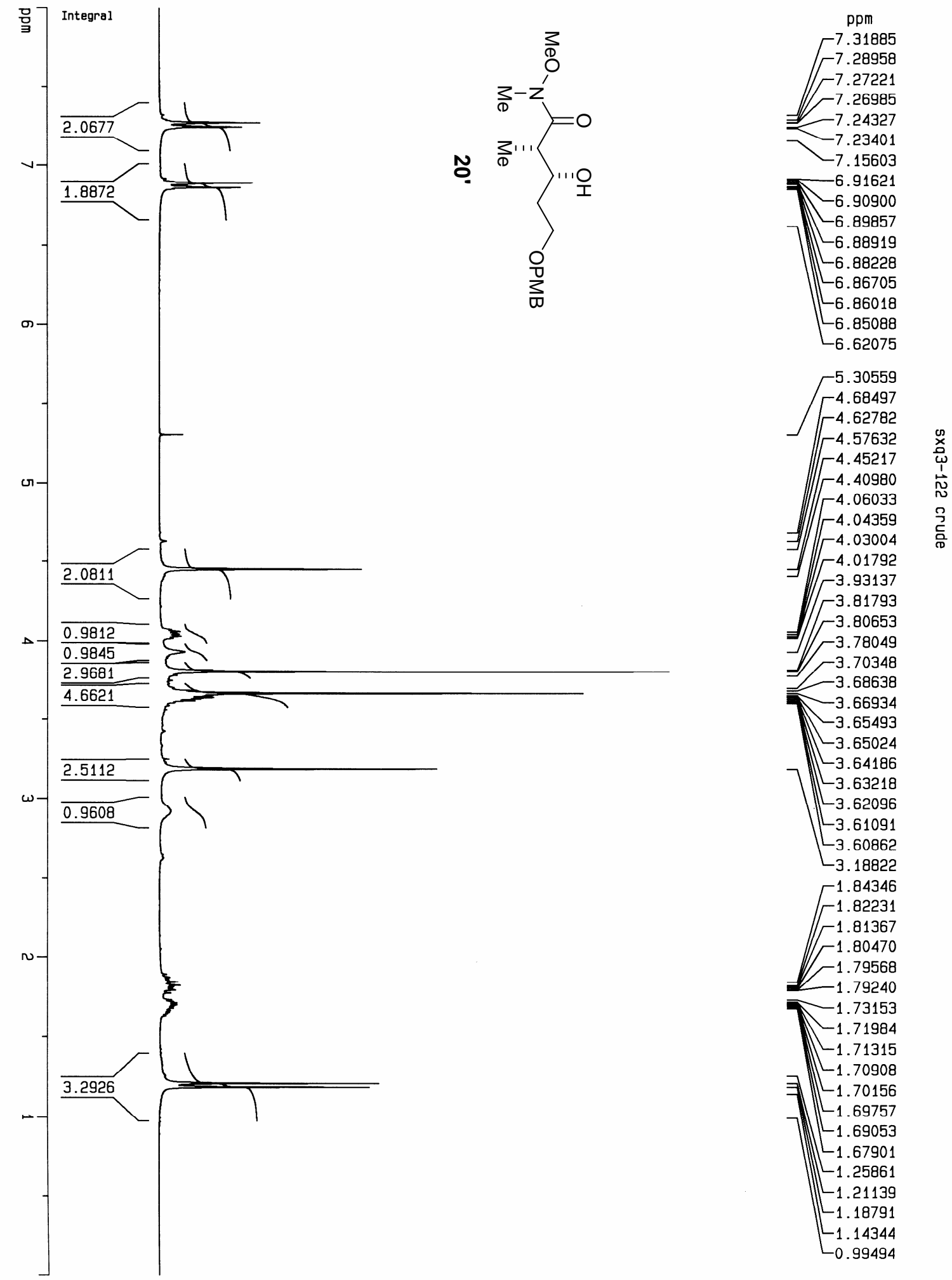

$-4.01792$

-3.93137

-3.81793

$-3.80653$

$-3.78049$

$-3.70348$

$-3.68638$

$-3.66934$

$-3.65493$

$-3.65024$

$-3.64186$

$-3.63218$

$-3.62096$

$-3.61091$

$L_{3.60862}$

$-3.18822$

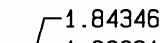

1.82231

$-1.81367$

$-1.80470$

$-1.79568$

$-1.79240$

$7-1.73153$

$-1.71984$

$-1.71315$

$L_{1.70908}$

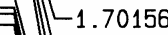

$L_{1} .69757$

$L_{1} .69053$

- 1.67901

$L_{1.25861}$

-1.21139

$L_{1.18791}$

$-1.14344$

$L_{0.99494}$ 

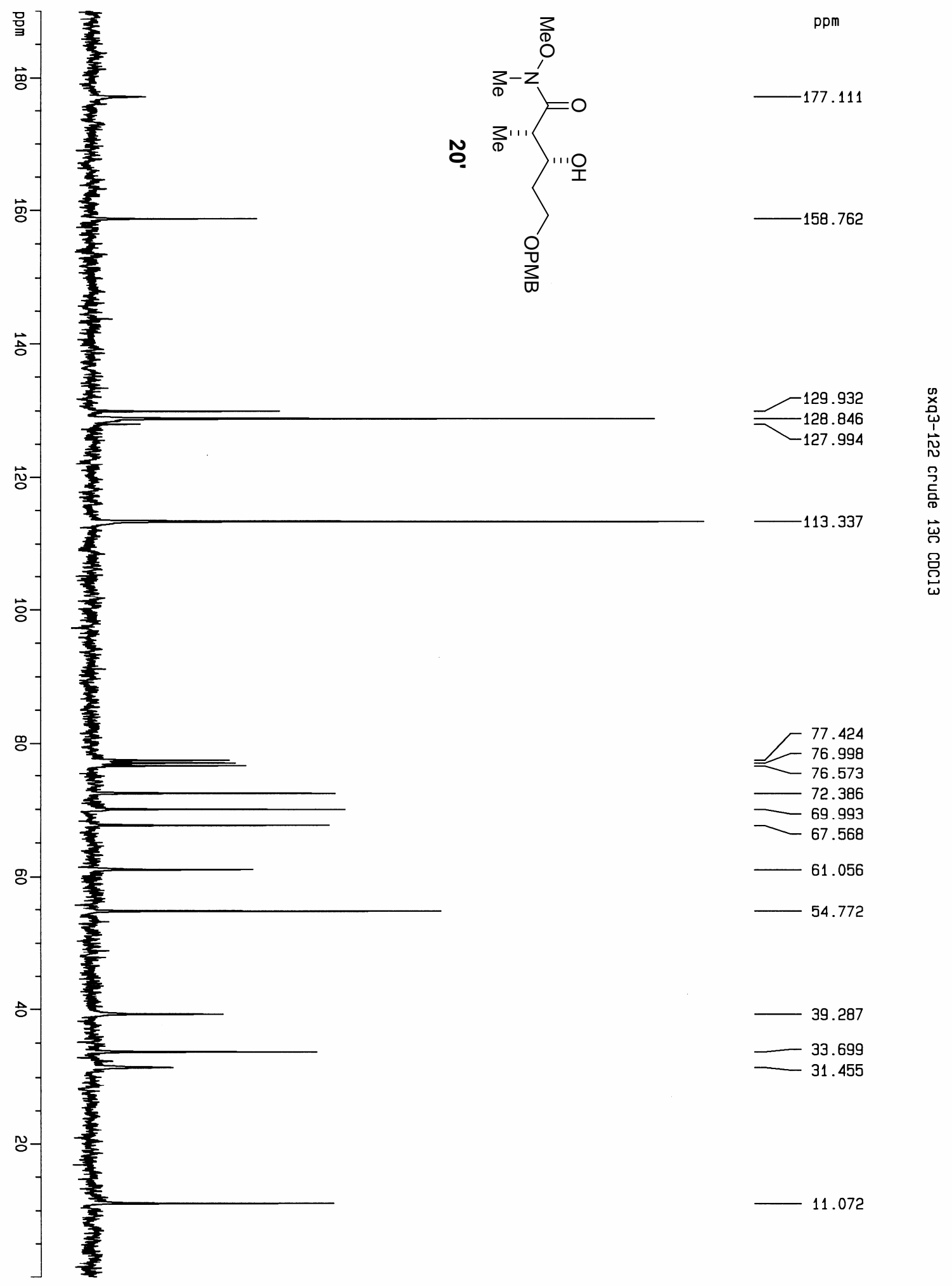

$-11.072$ 


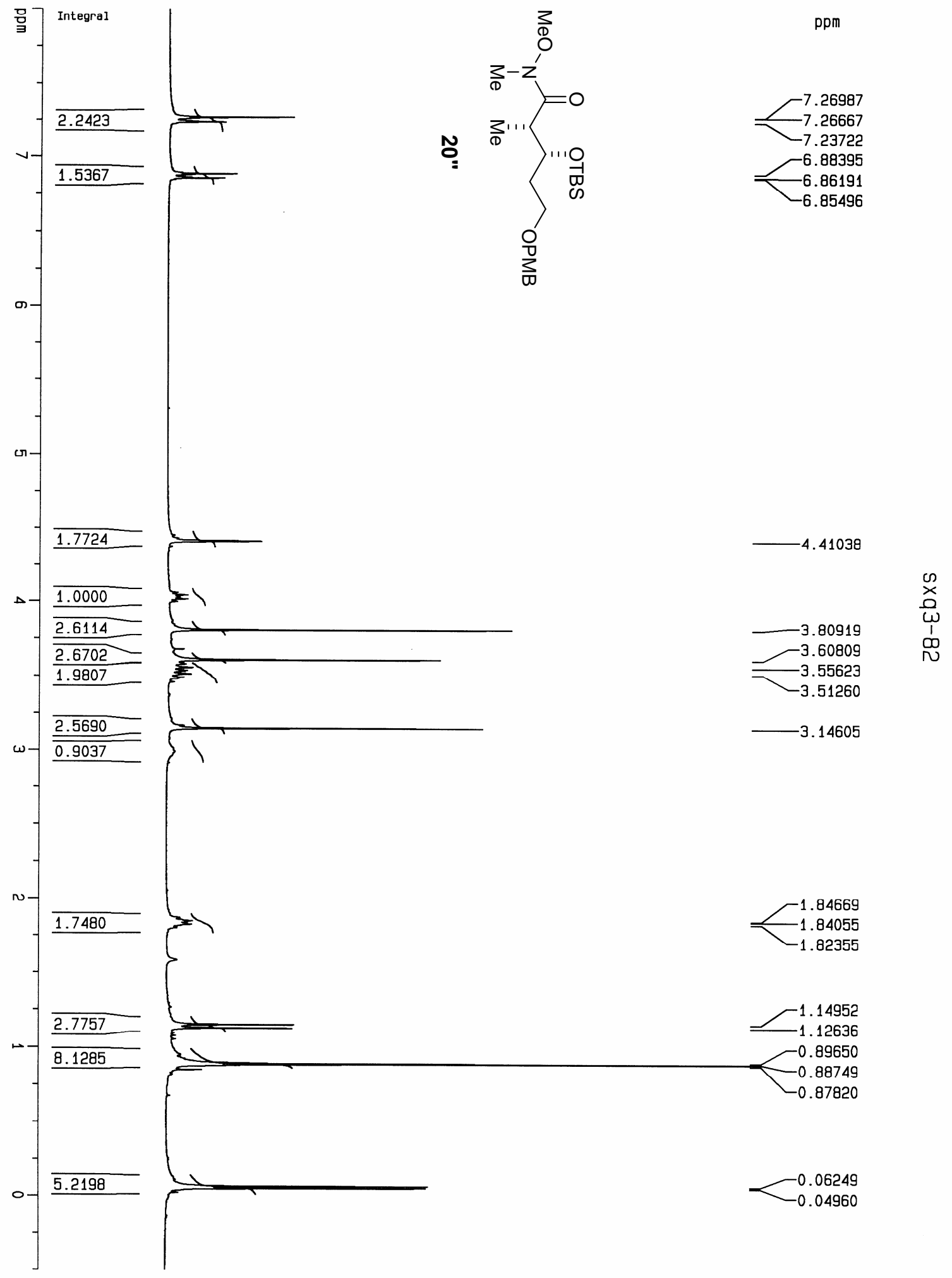




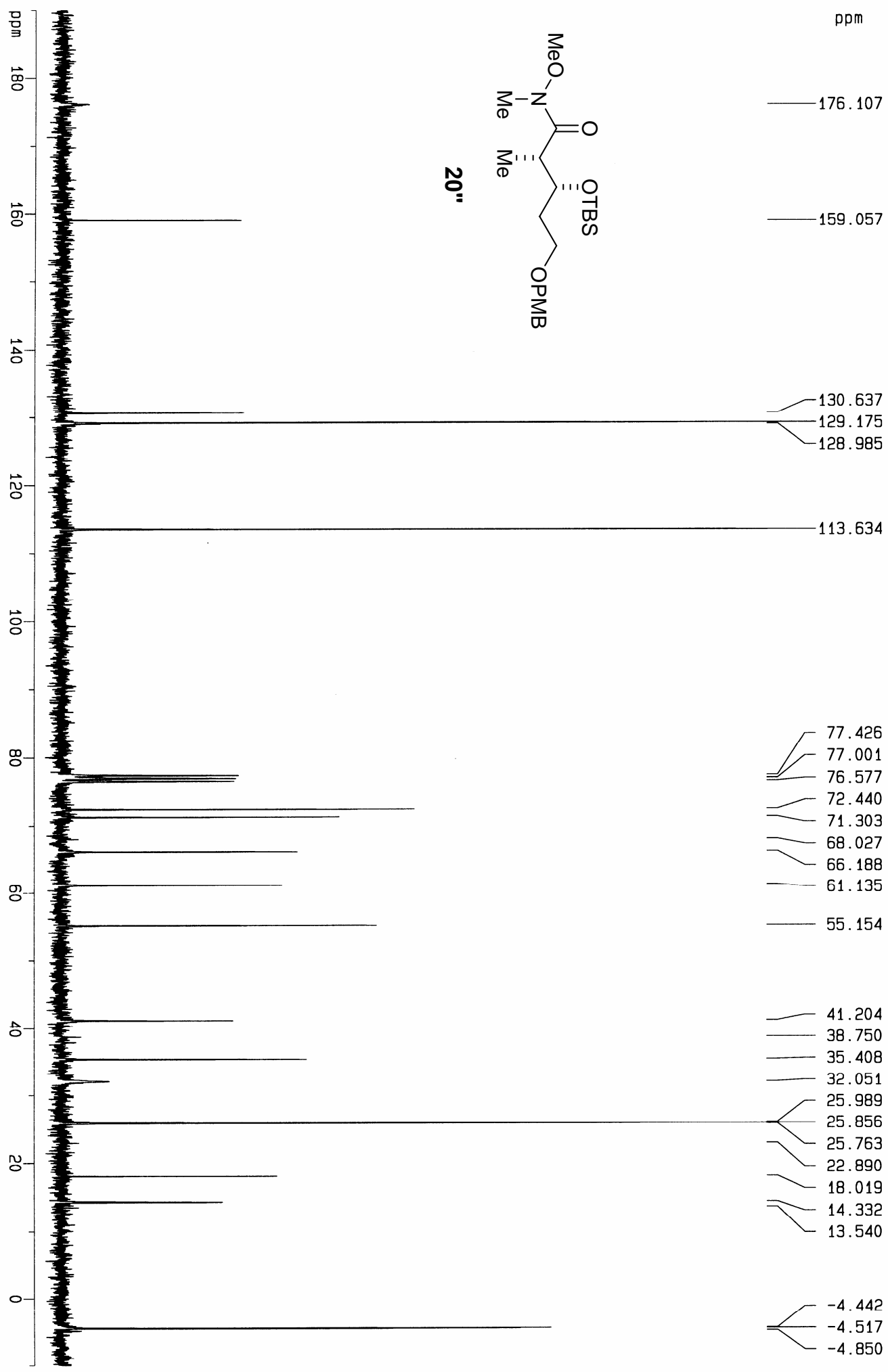




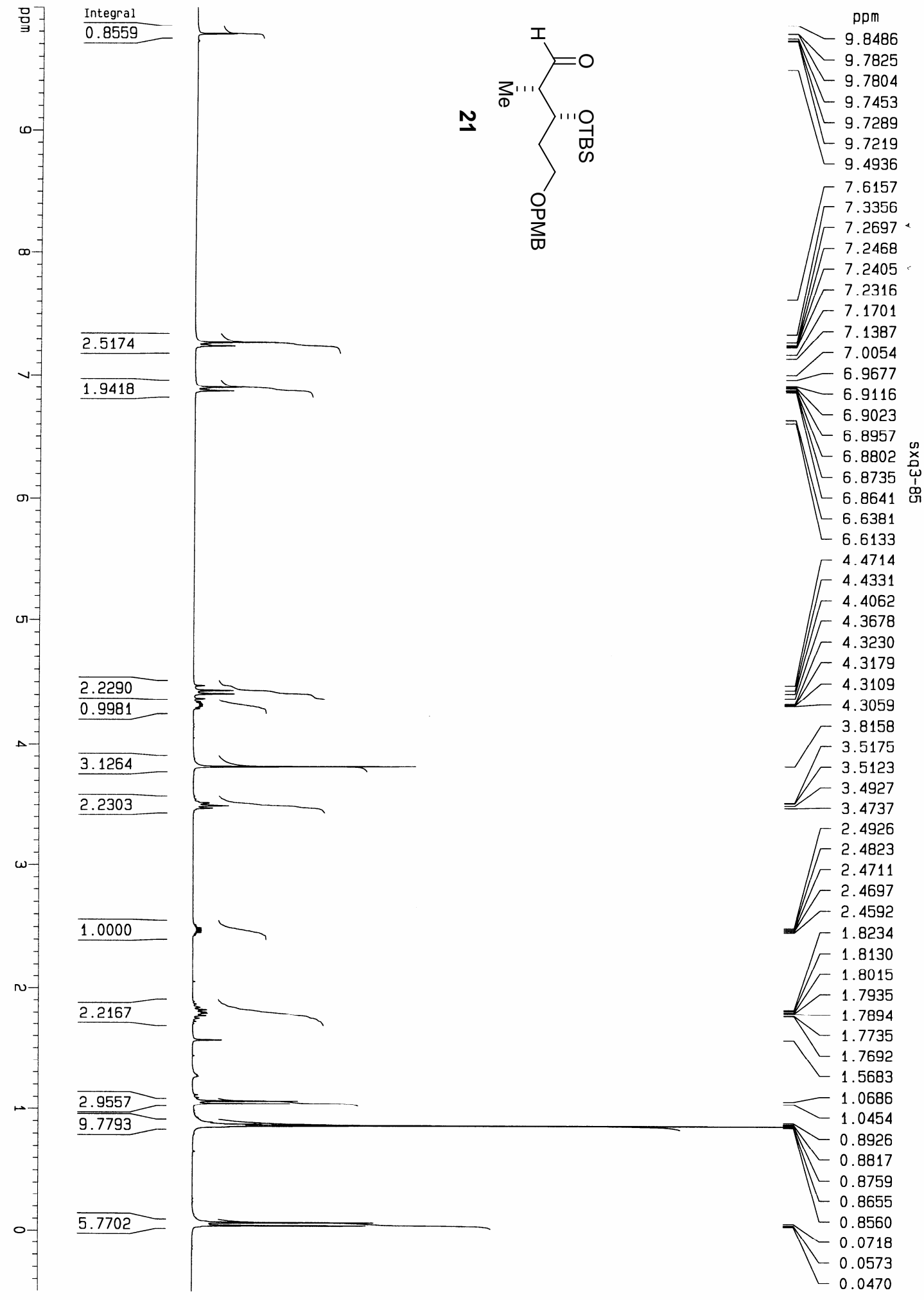




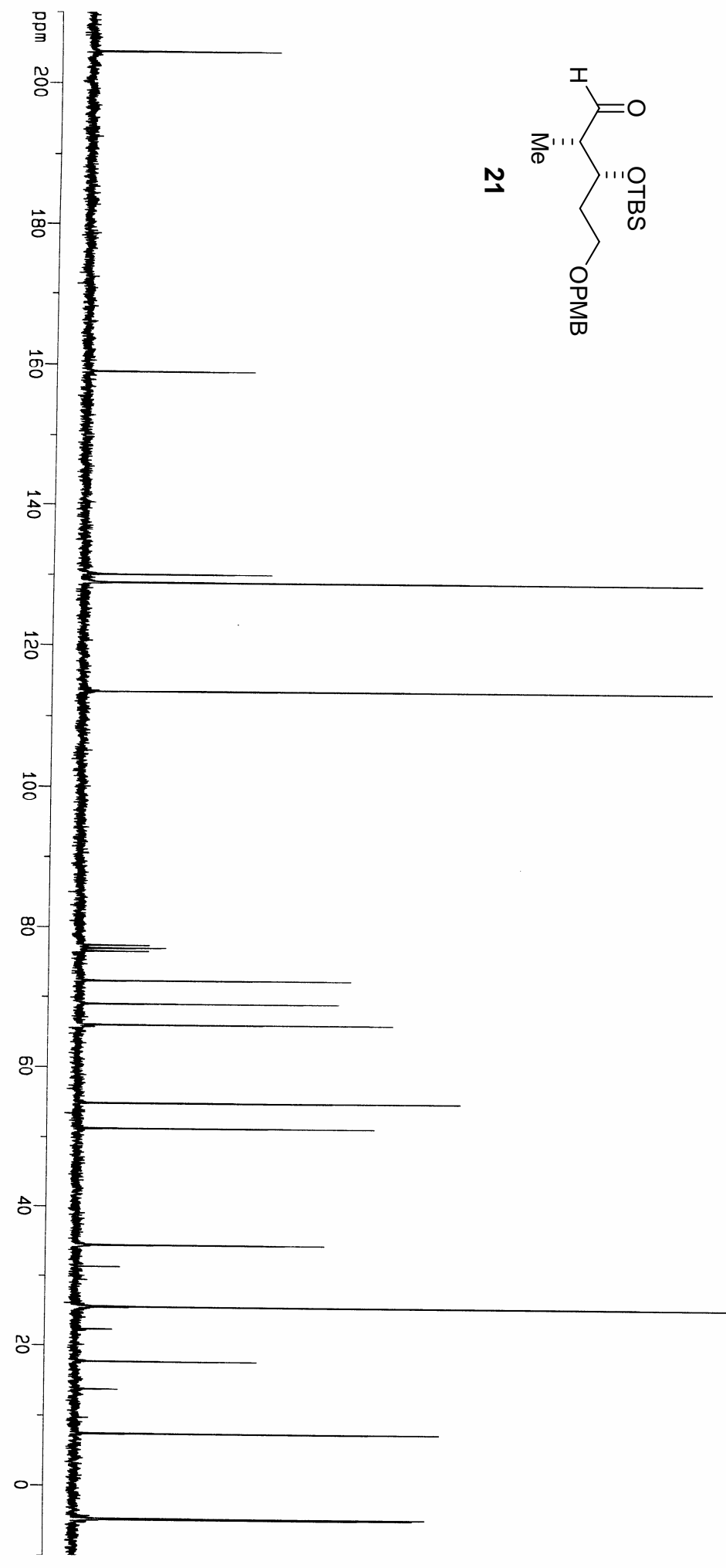

76.575

$=72.366$

69.001

$-65.946$

65.664

$\Gamma 54.833$

51.275

] 34.398

31.340

25.689

$-25.659$

- 25.627

25.596

$-25.527$

$\left[\begin{array}{r}25.527 \\ -25.436\end{array}\right.$

25.436

25.406

22.401

$-17.735$

$-13.878$

$-\quad 7.504$

$-4.357$

$\Gamma_{-}^{-4.655}$

$\begin{array}{r}-4.727 \\ -\quad-4.868 \\ \hline\end{array}$

$-4.970$ 


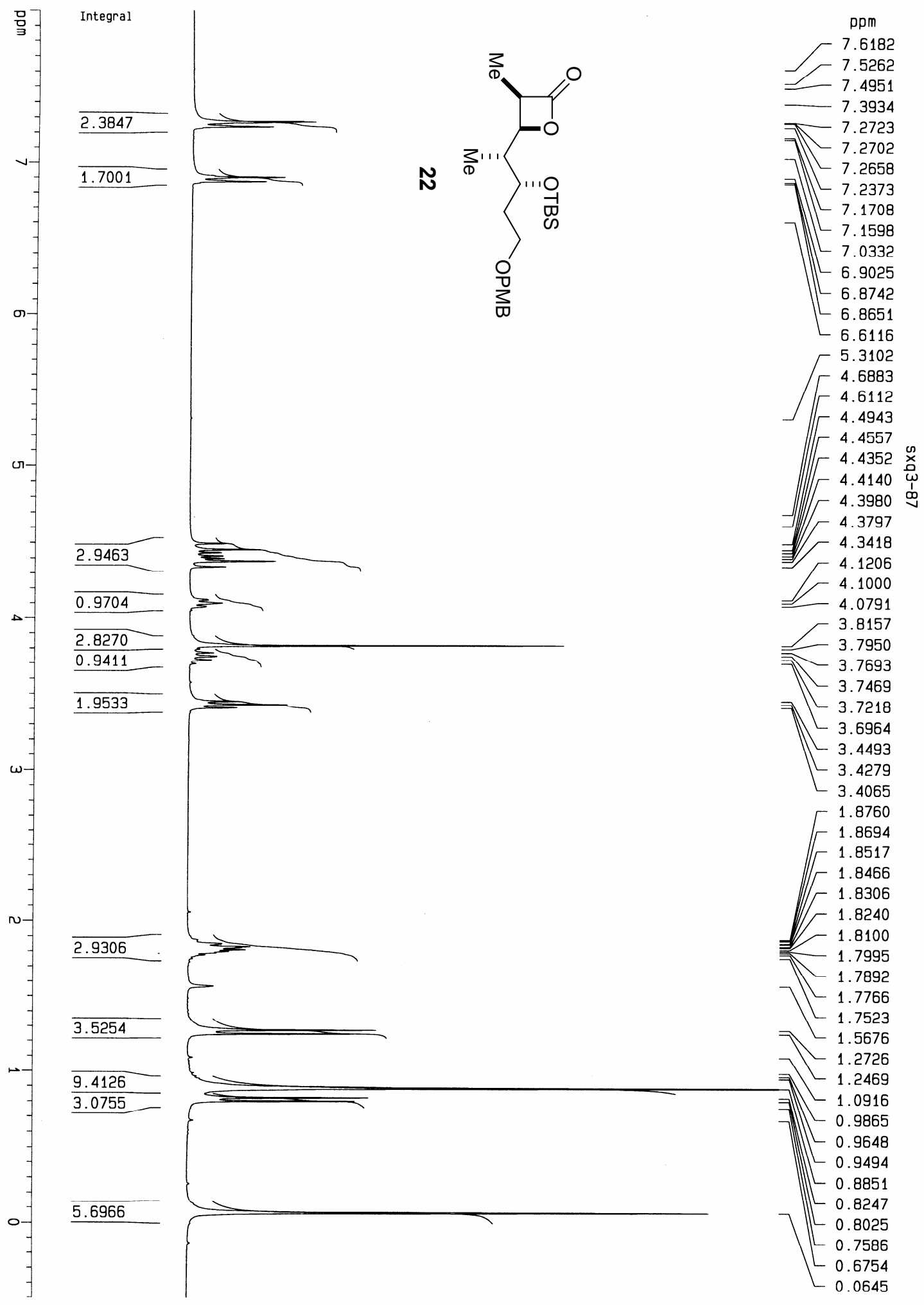



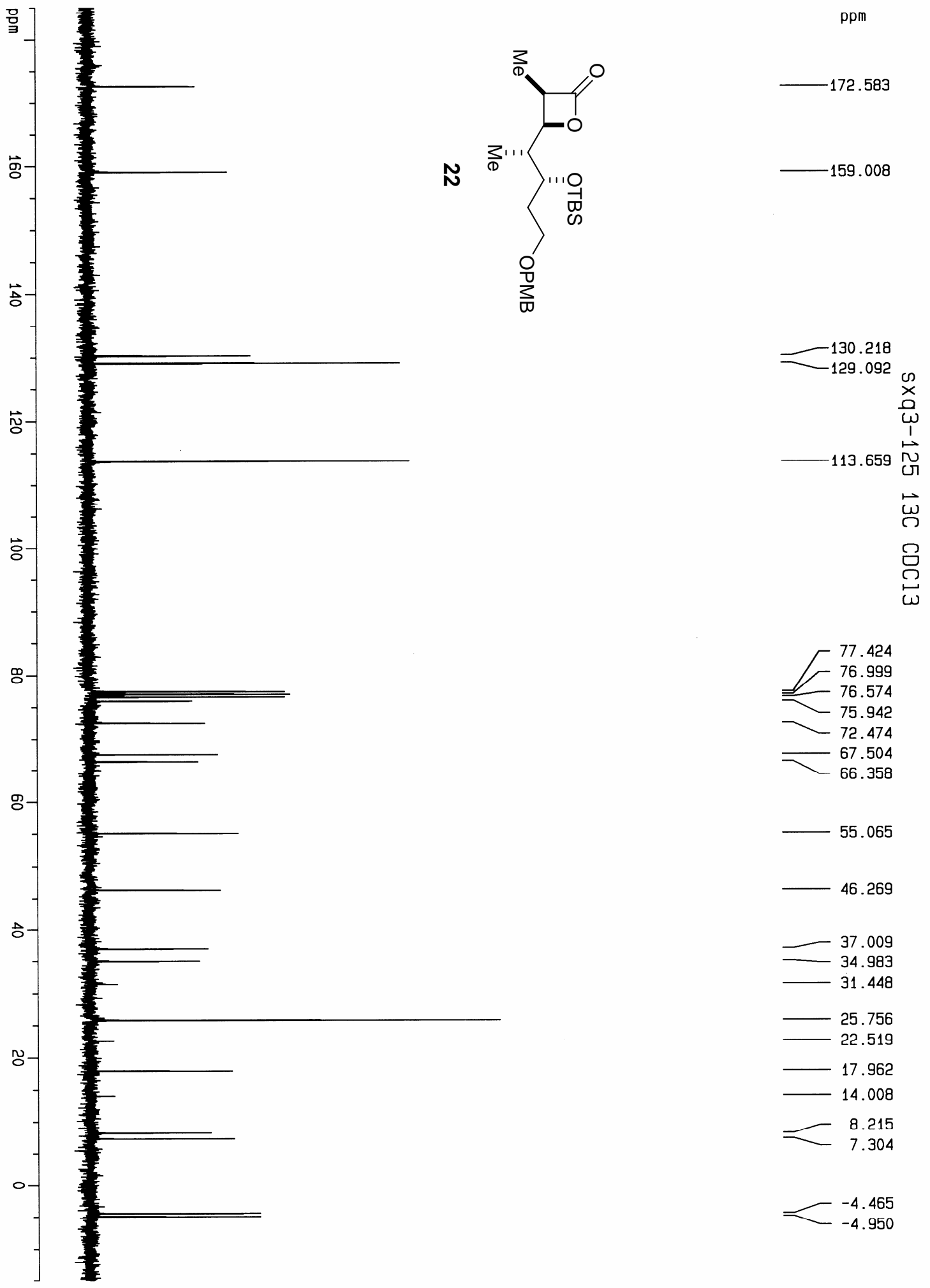


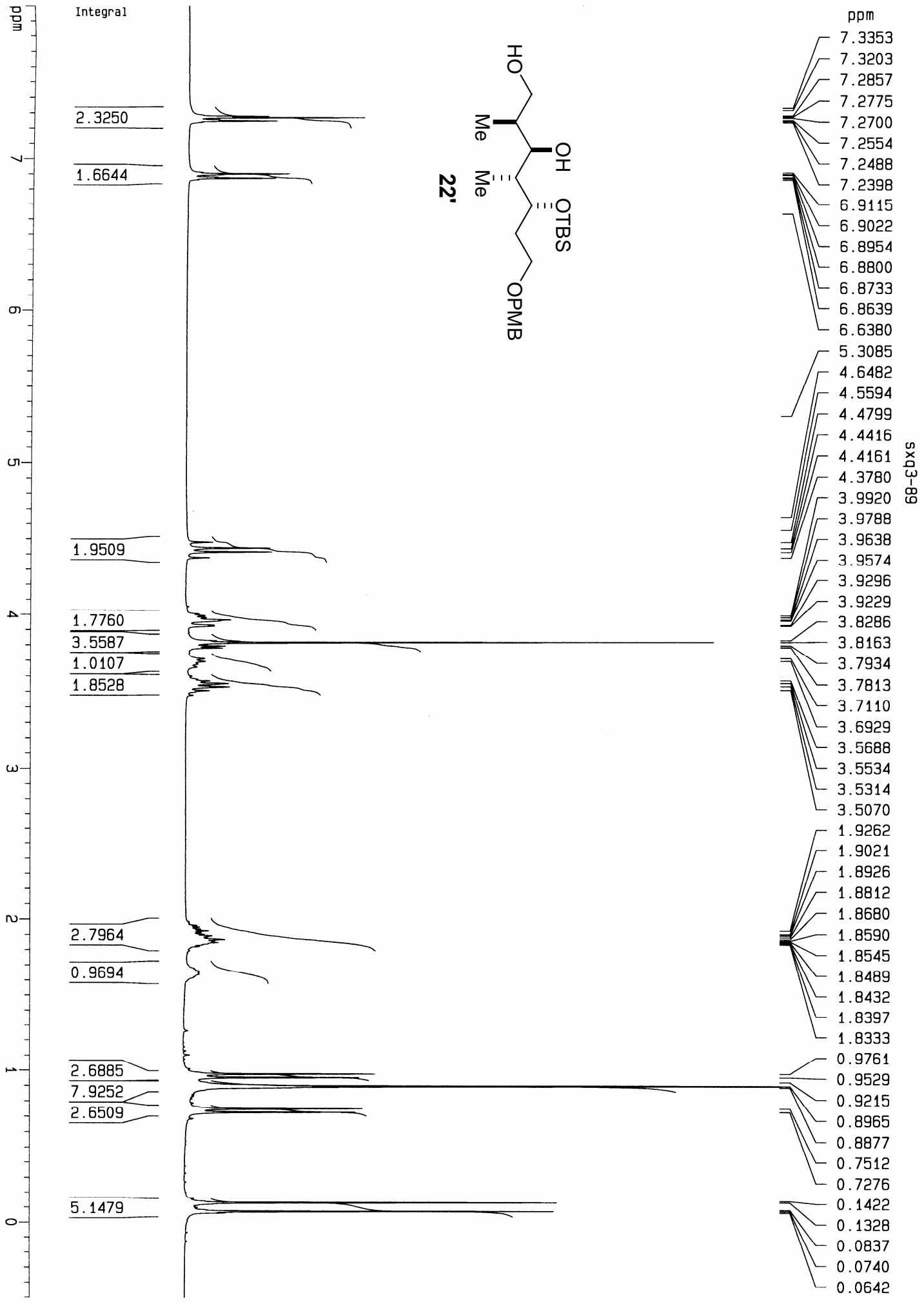



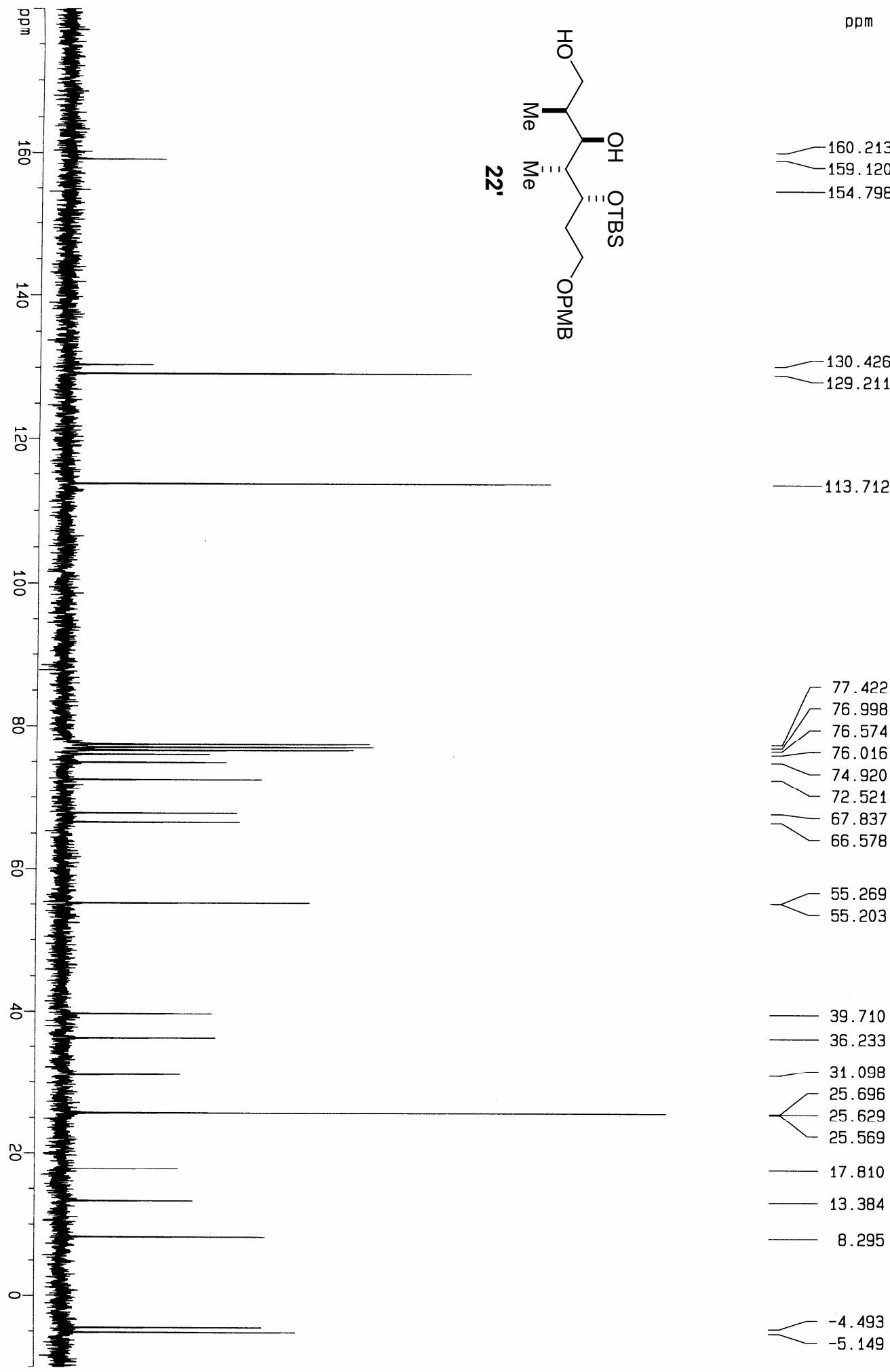


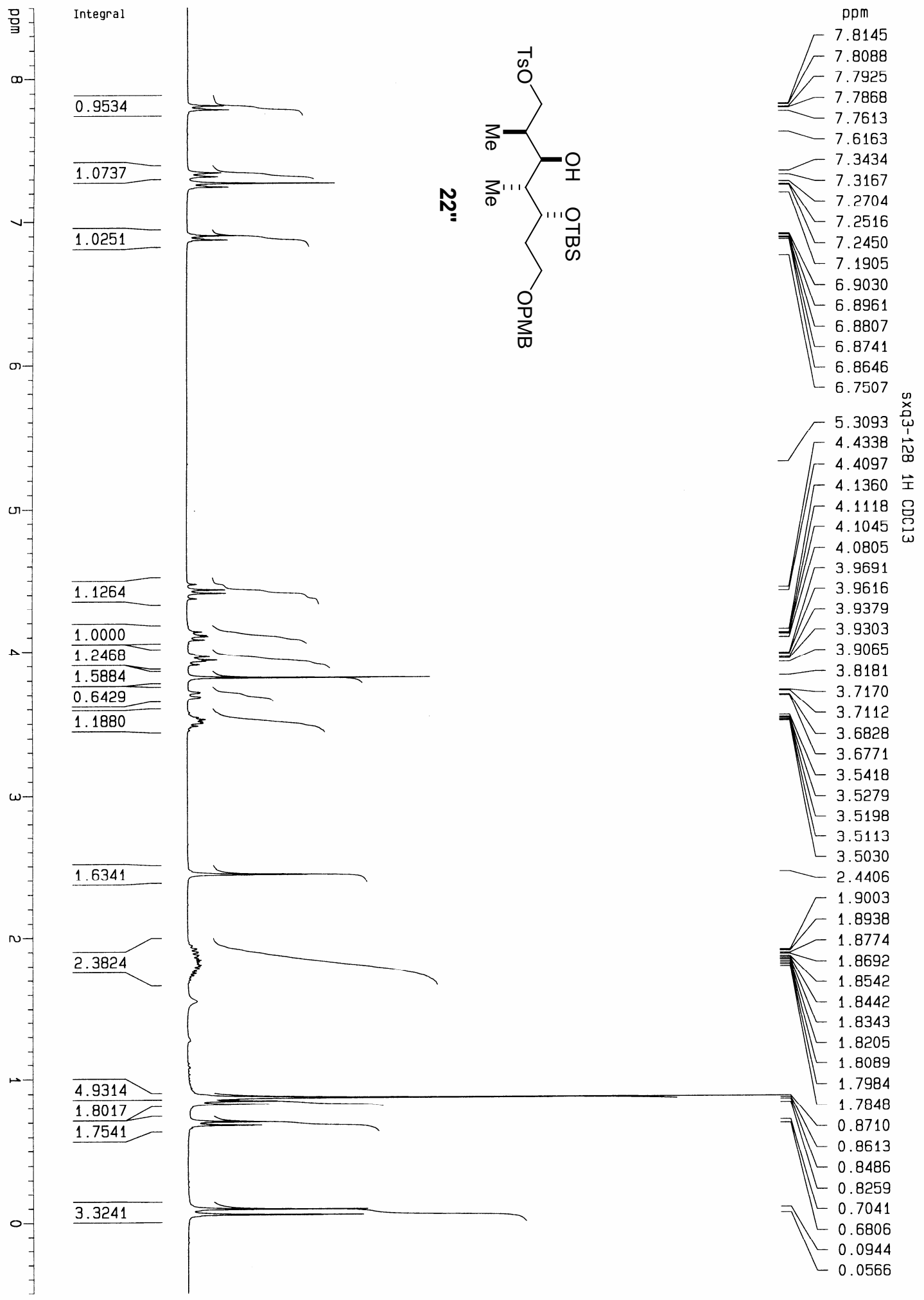



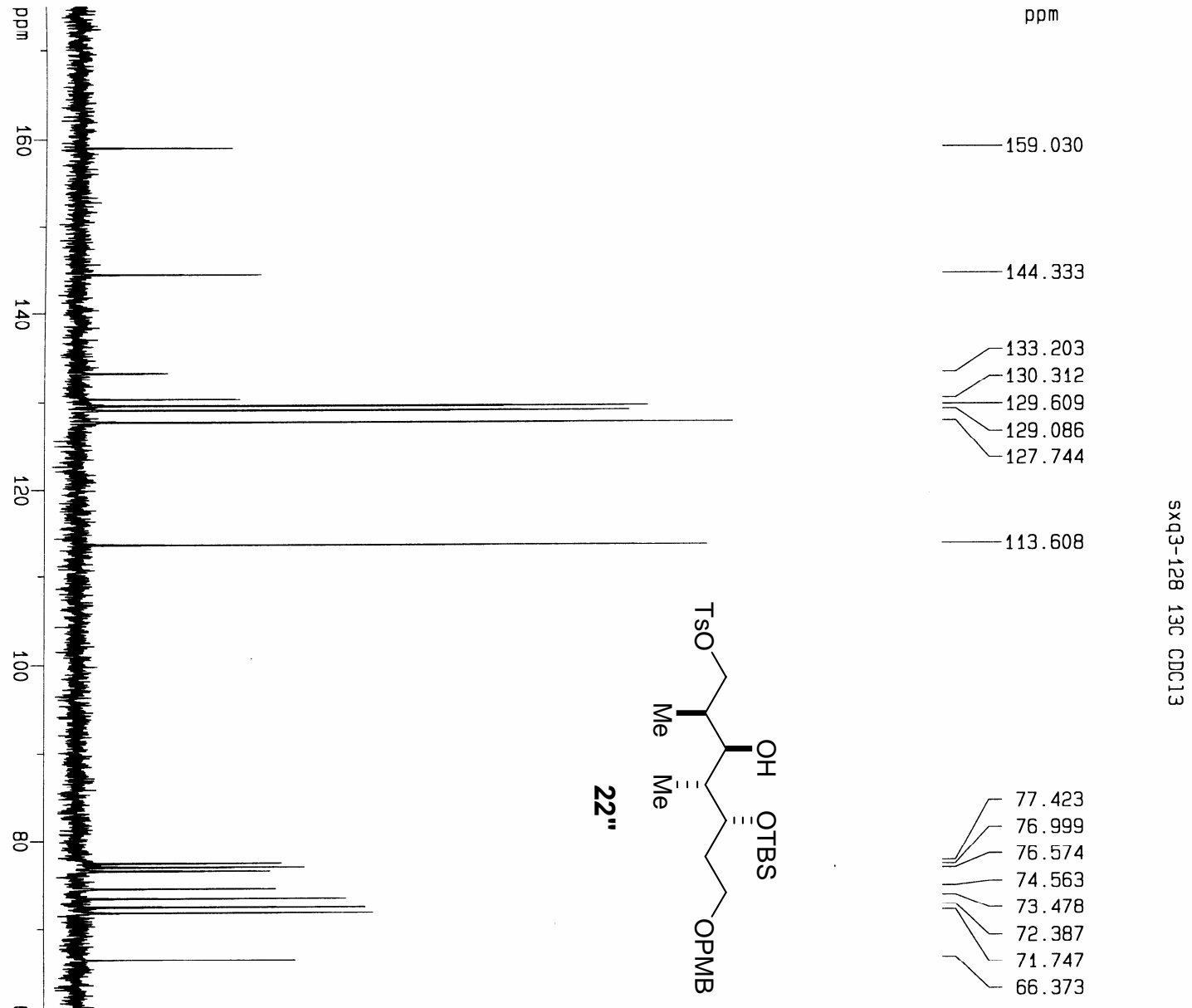

o

o

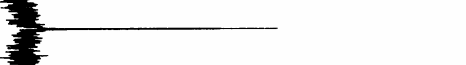

39.323

35.213

31.046

25.585

21.404

ก-

-

17.677

13.037

8.076

o-

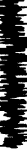

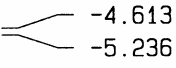




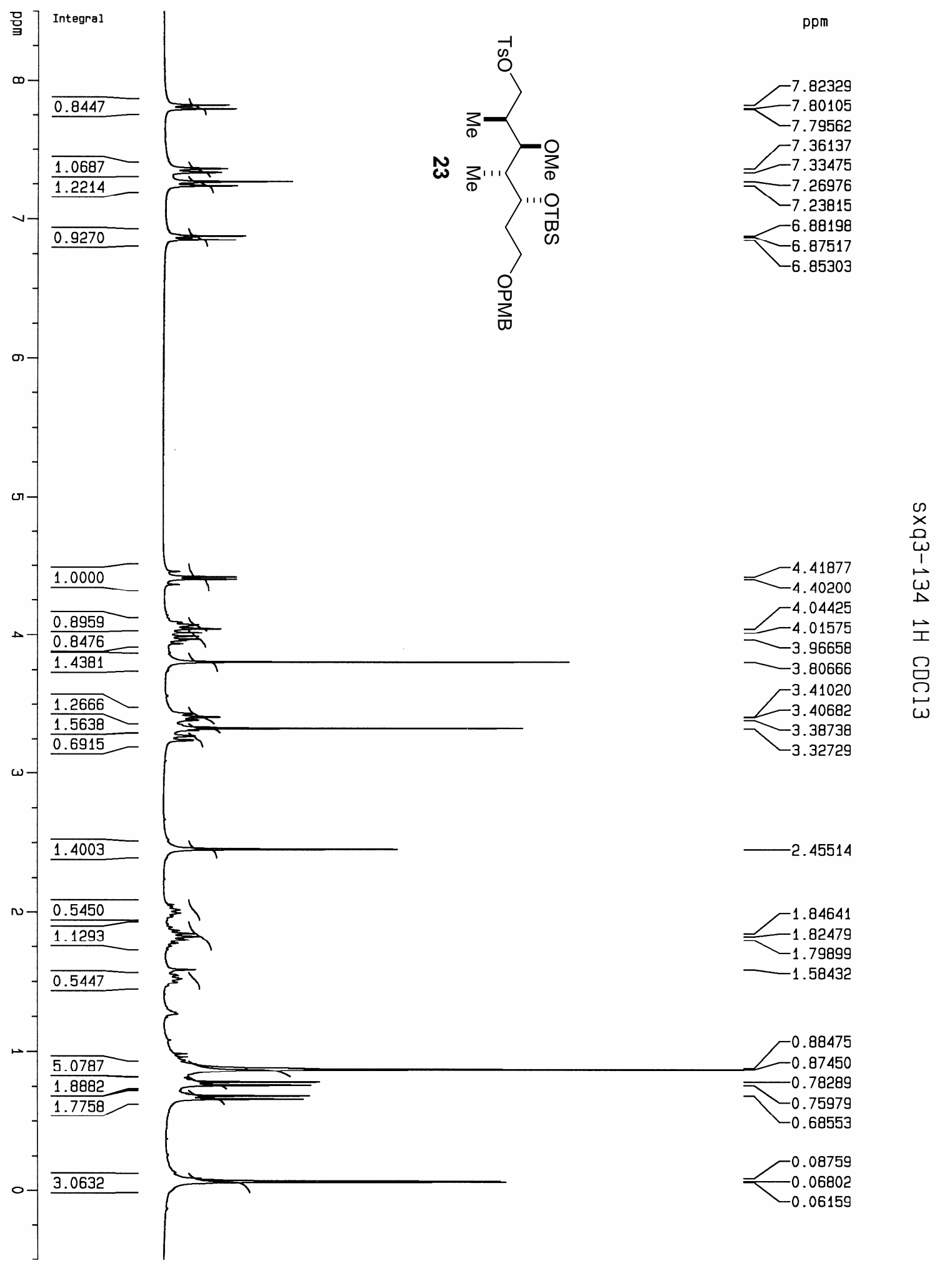



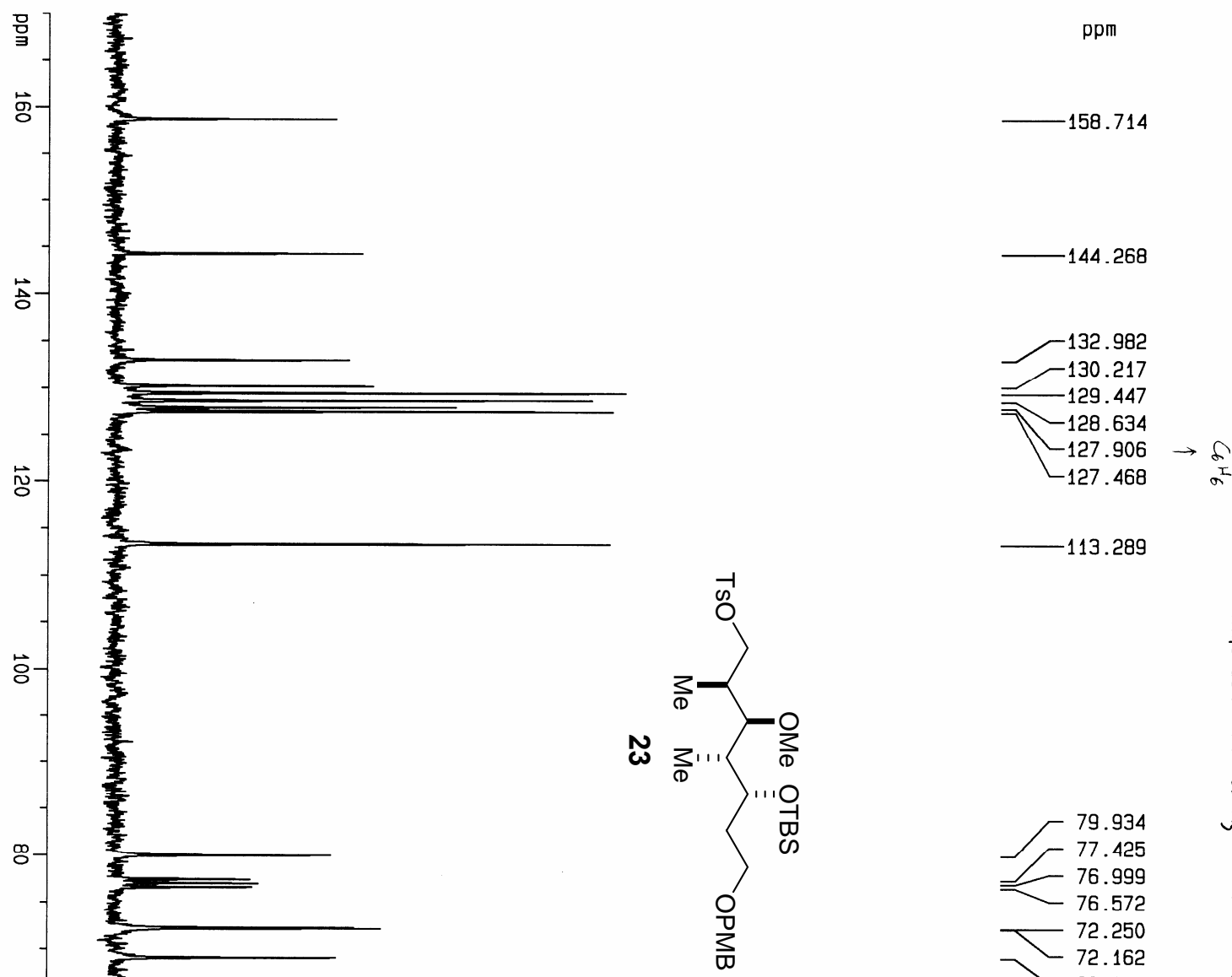

\&
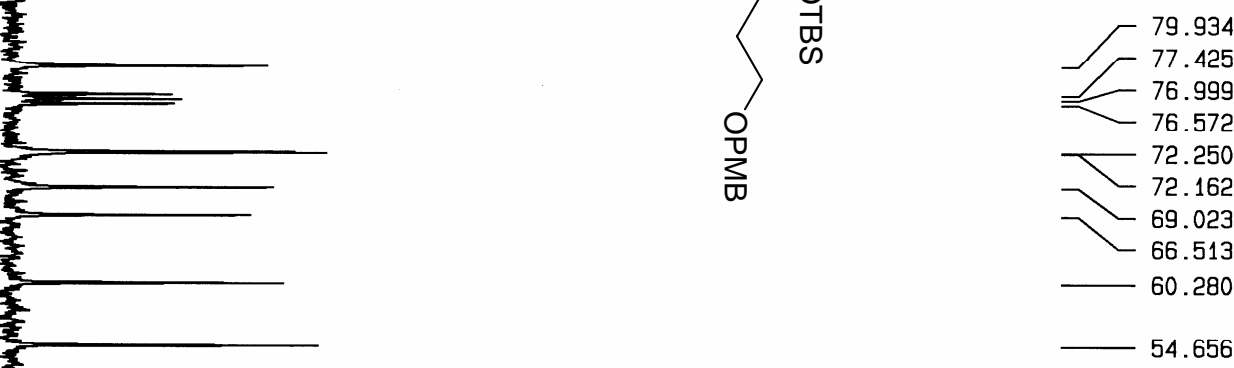

8
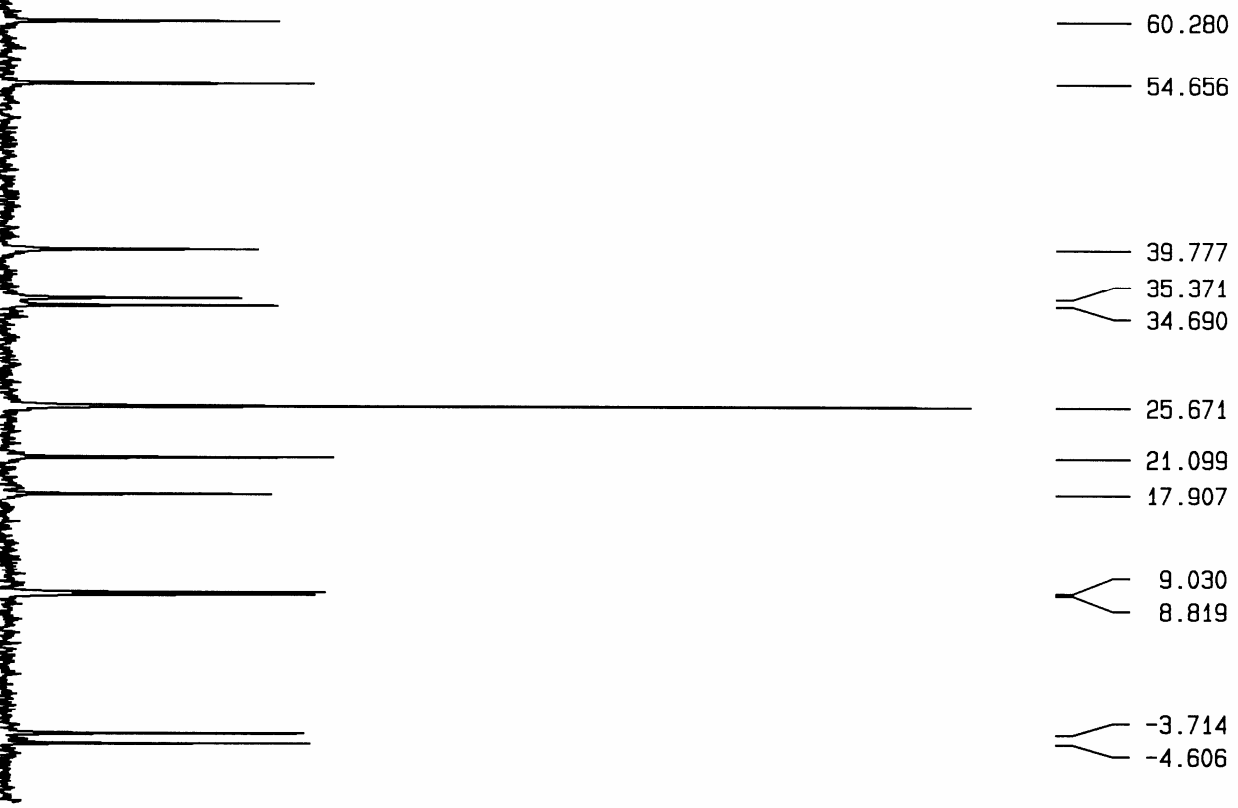

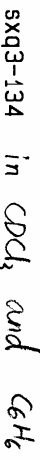

$\Delta$

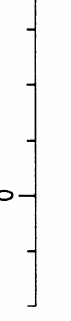




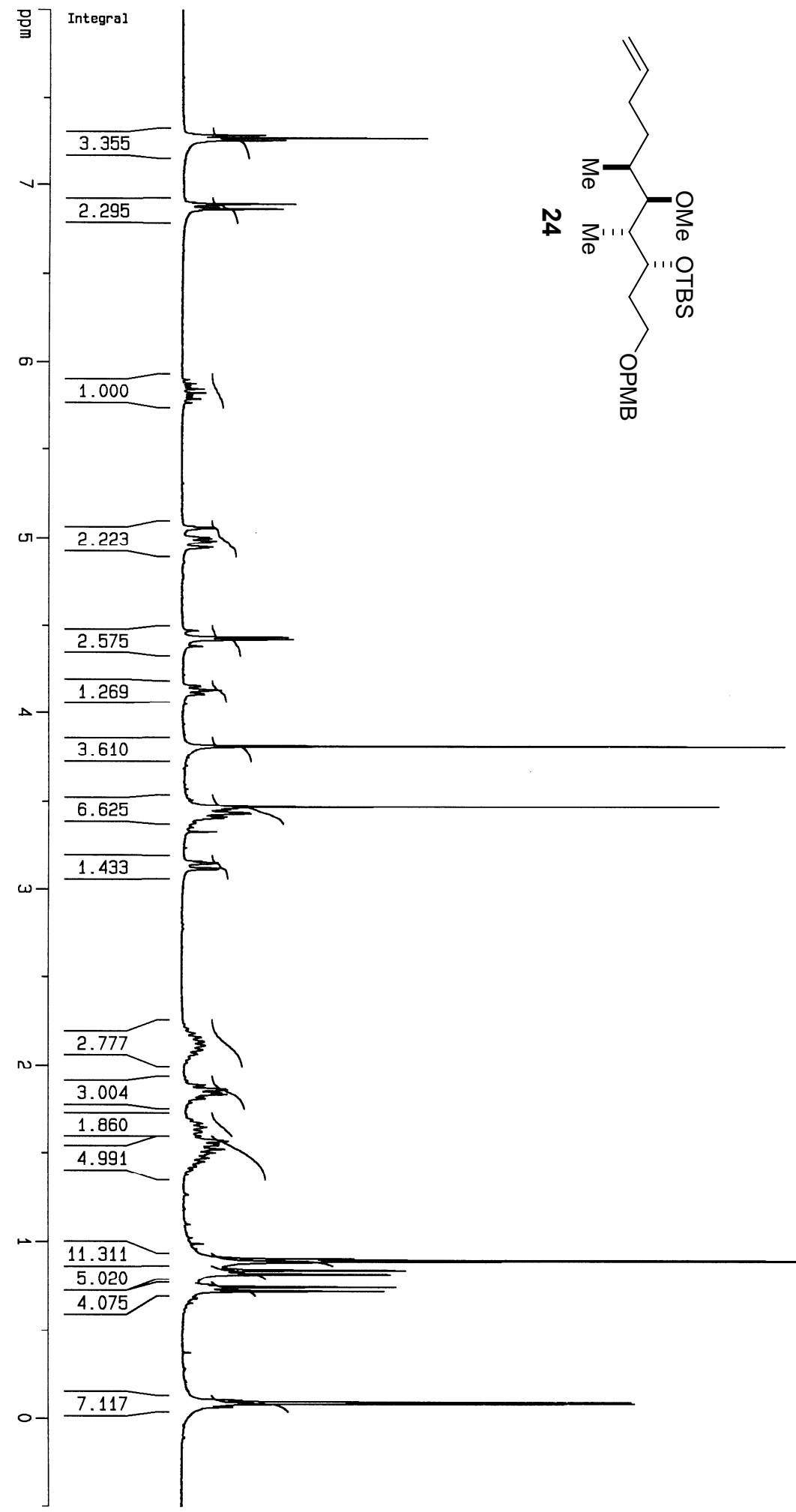

$\mathrm{ppm}$

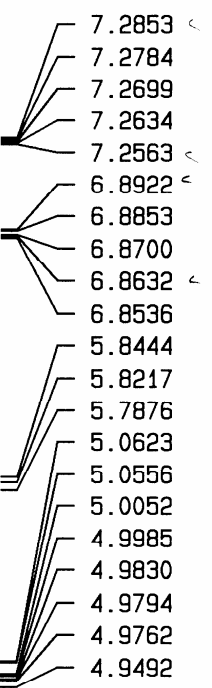

4.4337

F 4.4337

(

$\left[\begin{array}{l}4.1313 \\ 3.8123\end{array}\right.$

3.4768

$\sqrt{-3.4699}$

- 3.4592

- 3.4509

]-3.4342

$-3.4284$

$-3.4115$

3.4042

$-3.3273$

7.1515

- 3.1460

$-3.1200$

ᄂ 3.1145

Г 1.8644

F 1.8584

1.8437

- 1.8341

$\left[\begin{array}{l}1.5708 \\ 1.5654\end{array}\right.$

$-1.5514$

- 1.5420

$-1.5203$

$-1.5007$

$-1.4706$

$-0.9055$

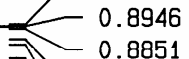

Ð 0.8851

$-0.8193$

$-0.7598$

$-0.7459$

$\simeq 0.7229$

$\sim 0.1044$

$-0.0907$

$-0.0831$

$-0.0633$ 

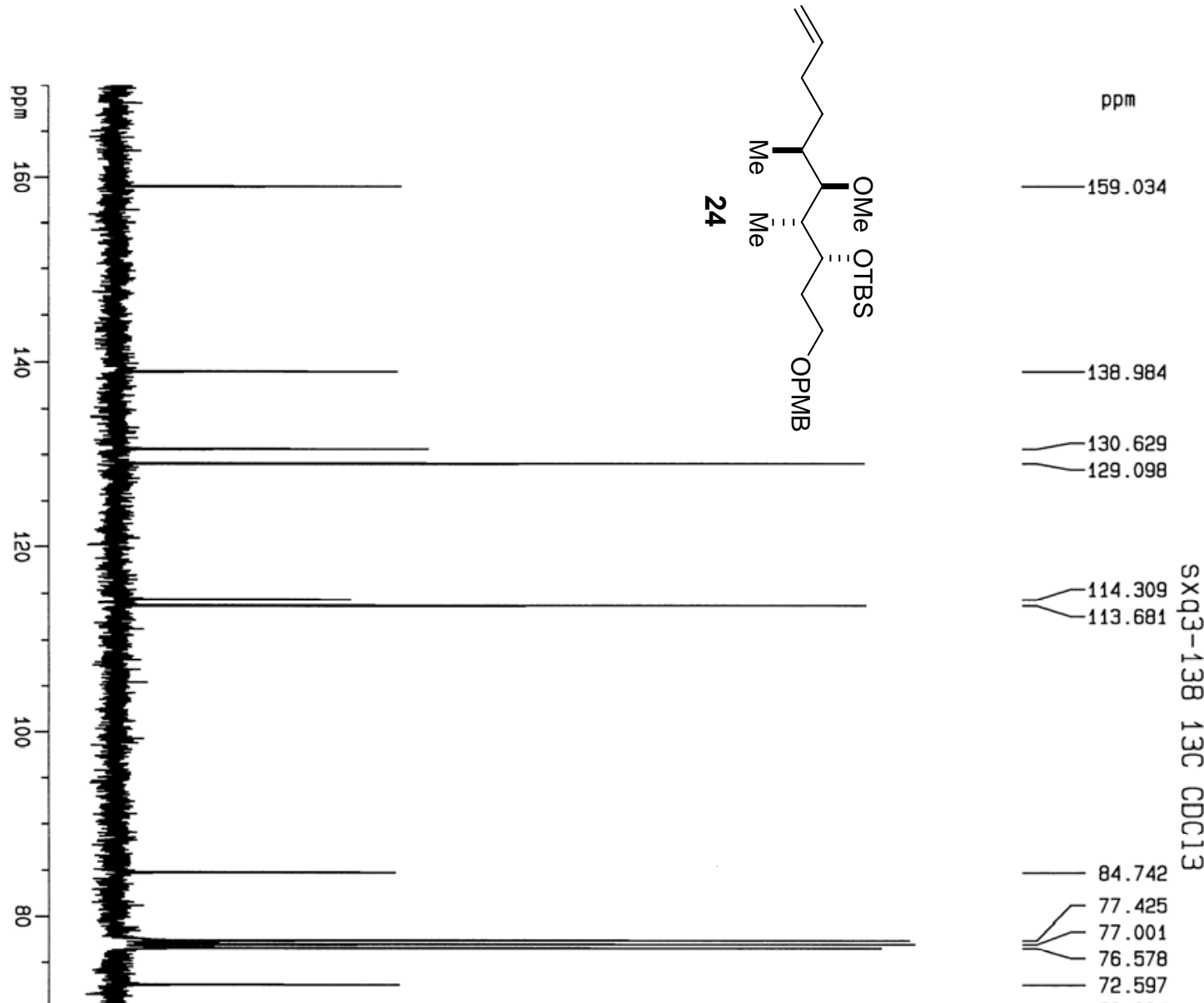

$\overrightarrow{\mathrm{\omega}}$

음

:

of

ô-

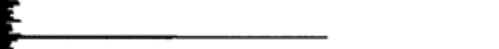

$-40.707$

35.938

34.388

31.964

25.994

18. 296

12.774

9.475

o-

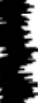




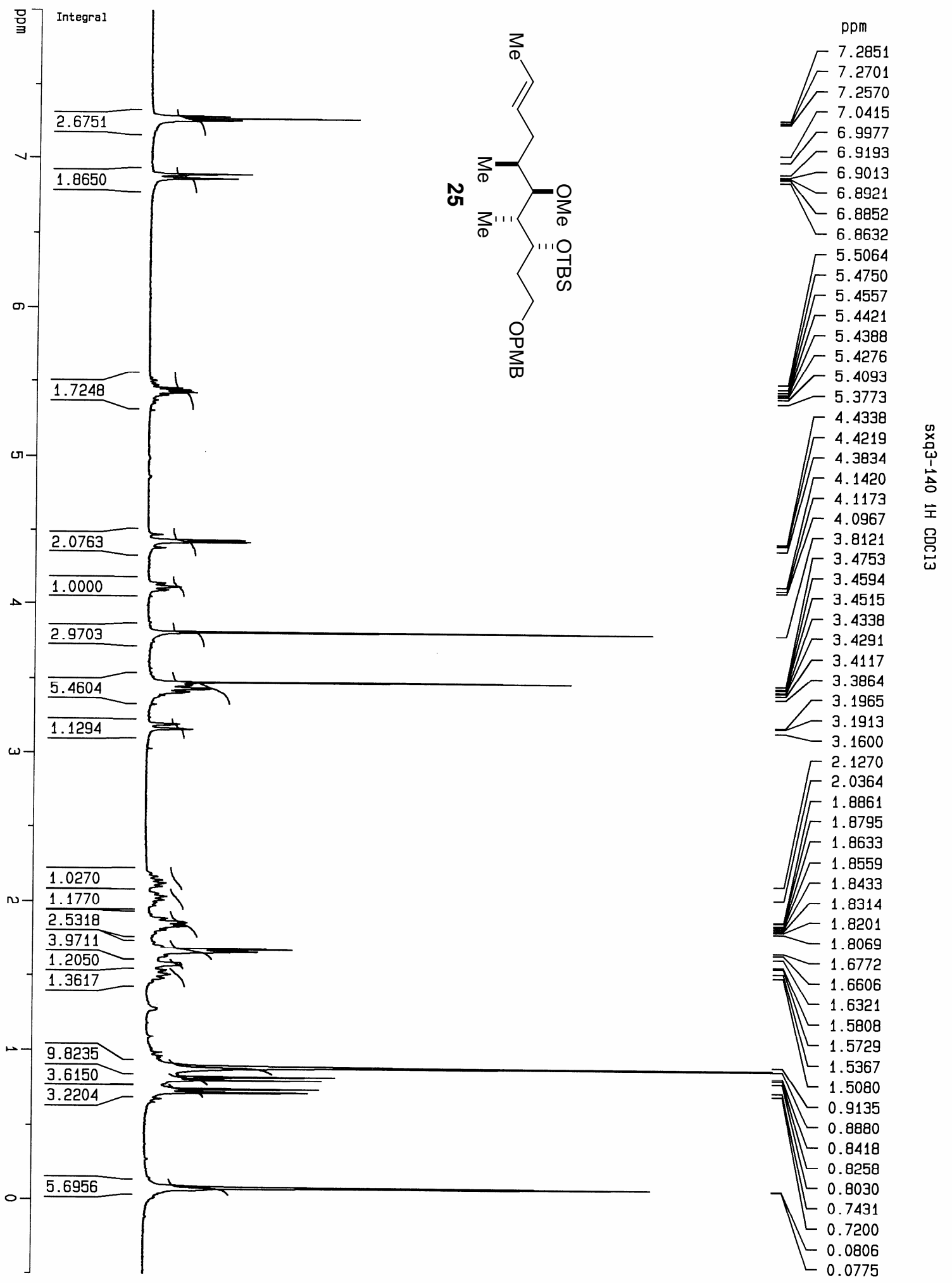



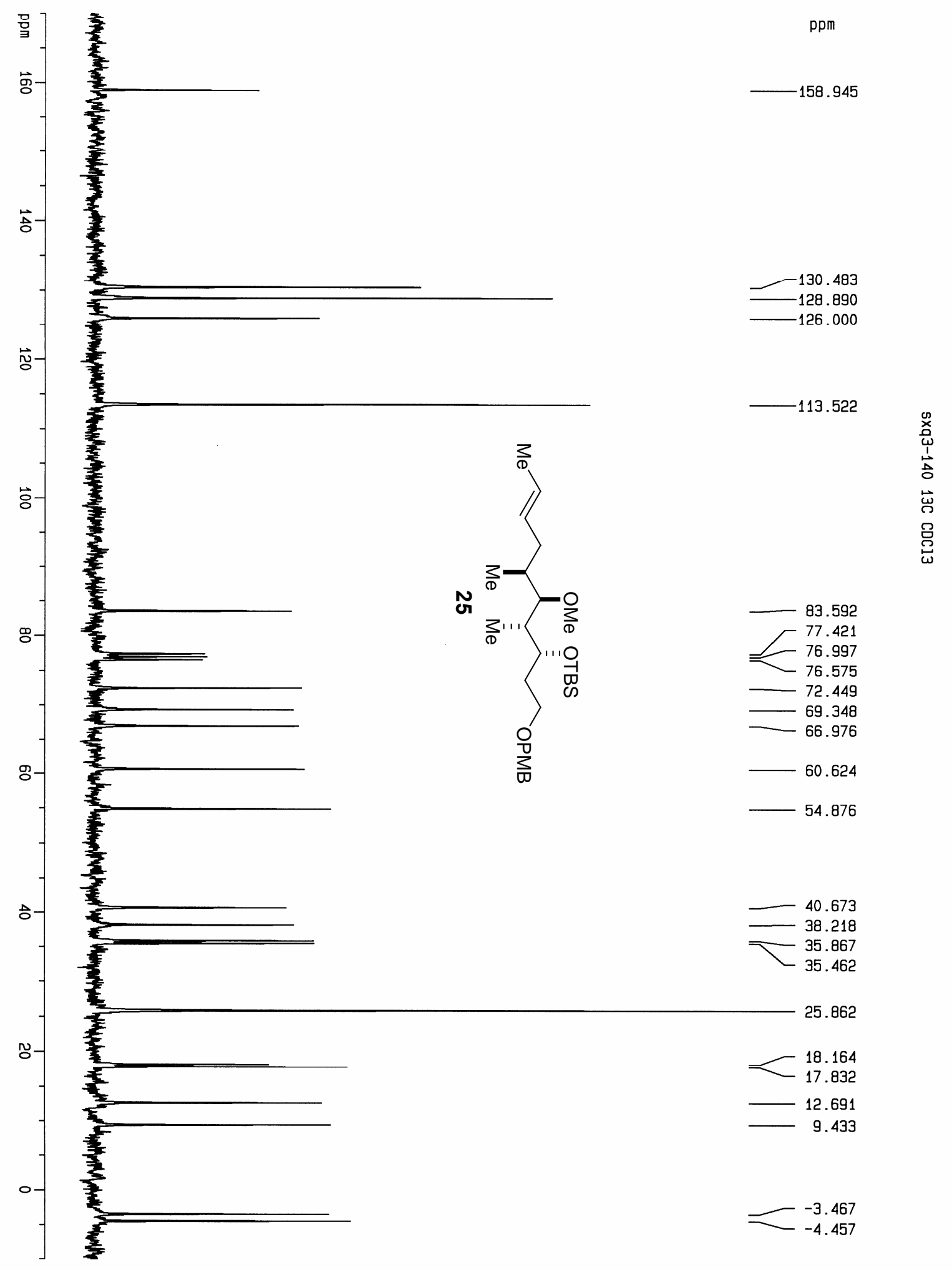


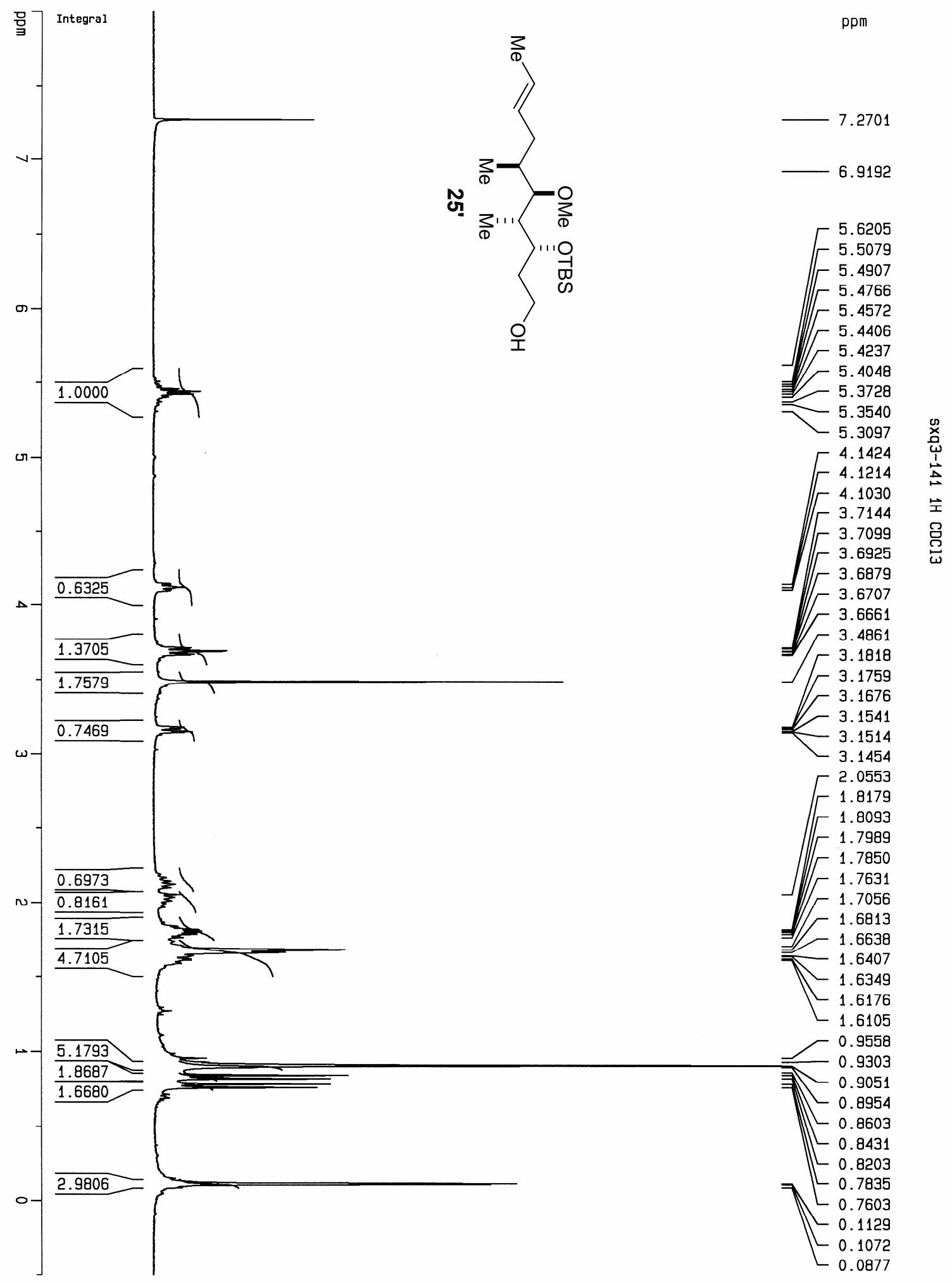




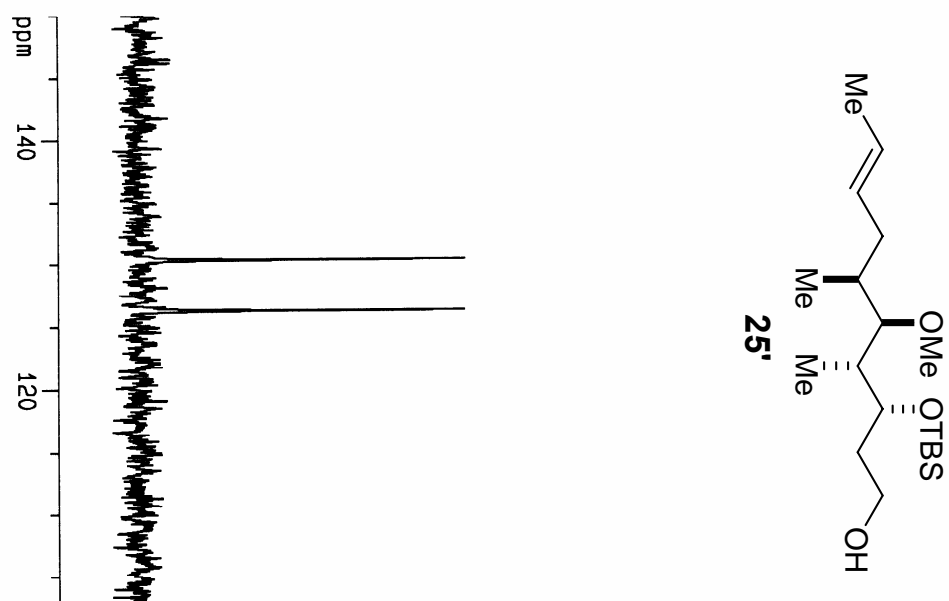

$\mathrm{ppm}$

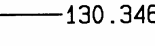

$-126.291$

을

甲

章
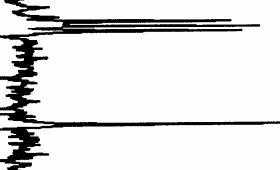

69.167

g

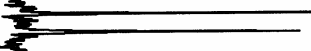

$-60.831$

59.367

응

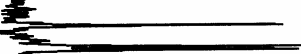

$-40.167$

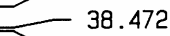

38.228

$\sim 35.458$

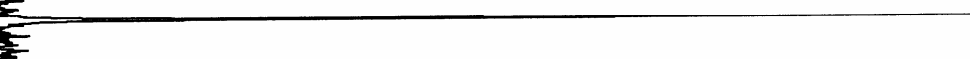

25.895

잉

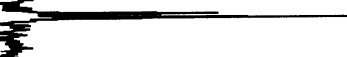

18.208

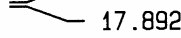

12.644

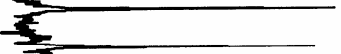

$-9.606$

o-

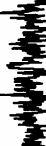

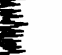




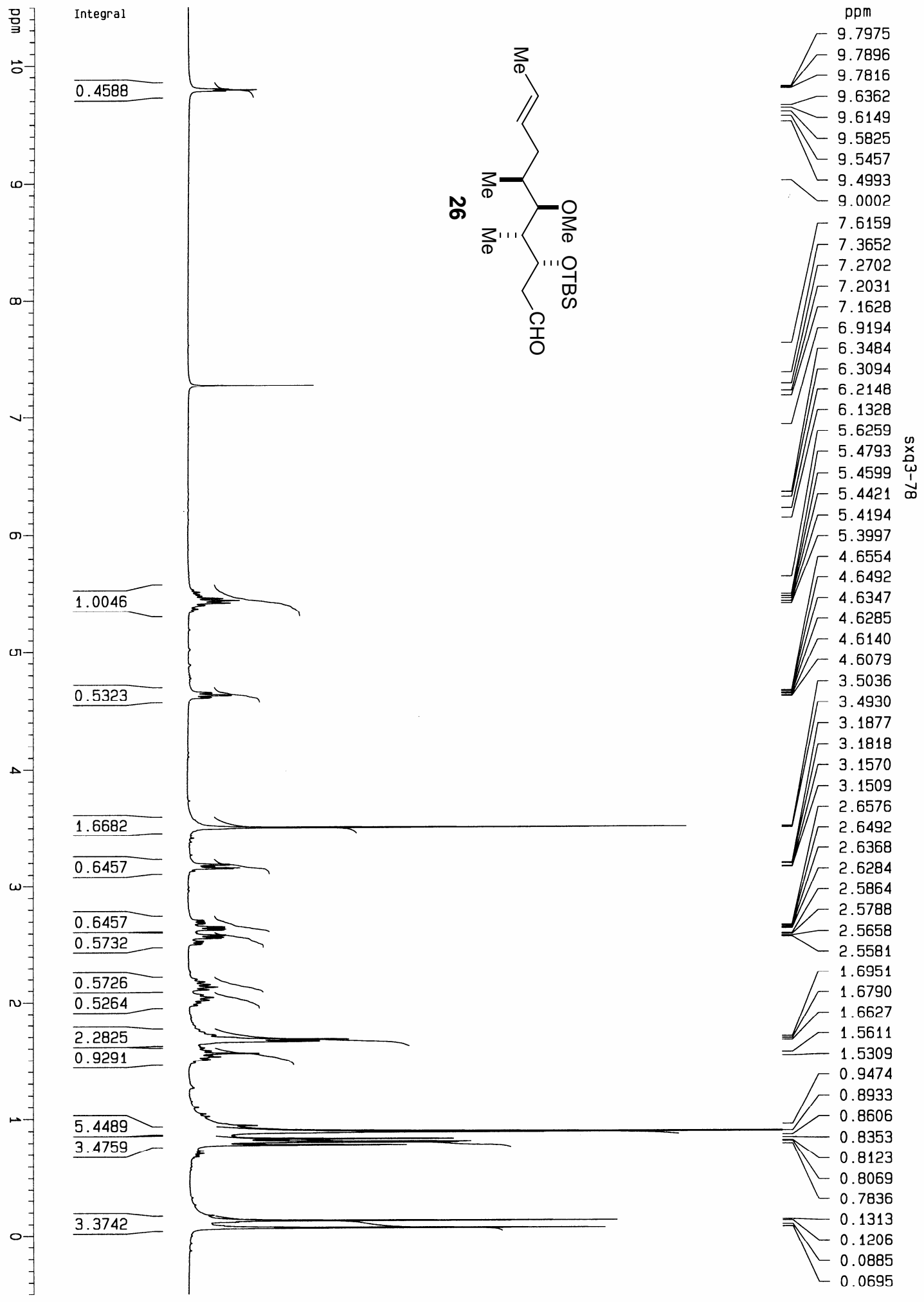



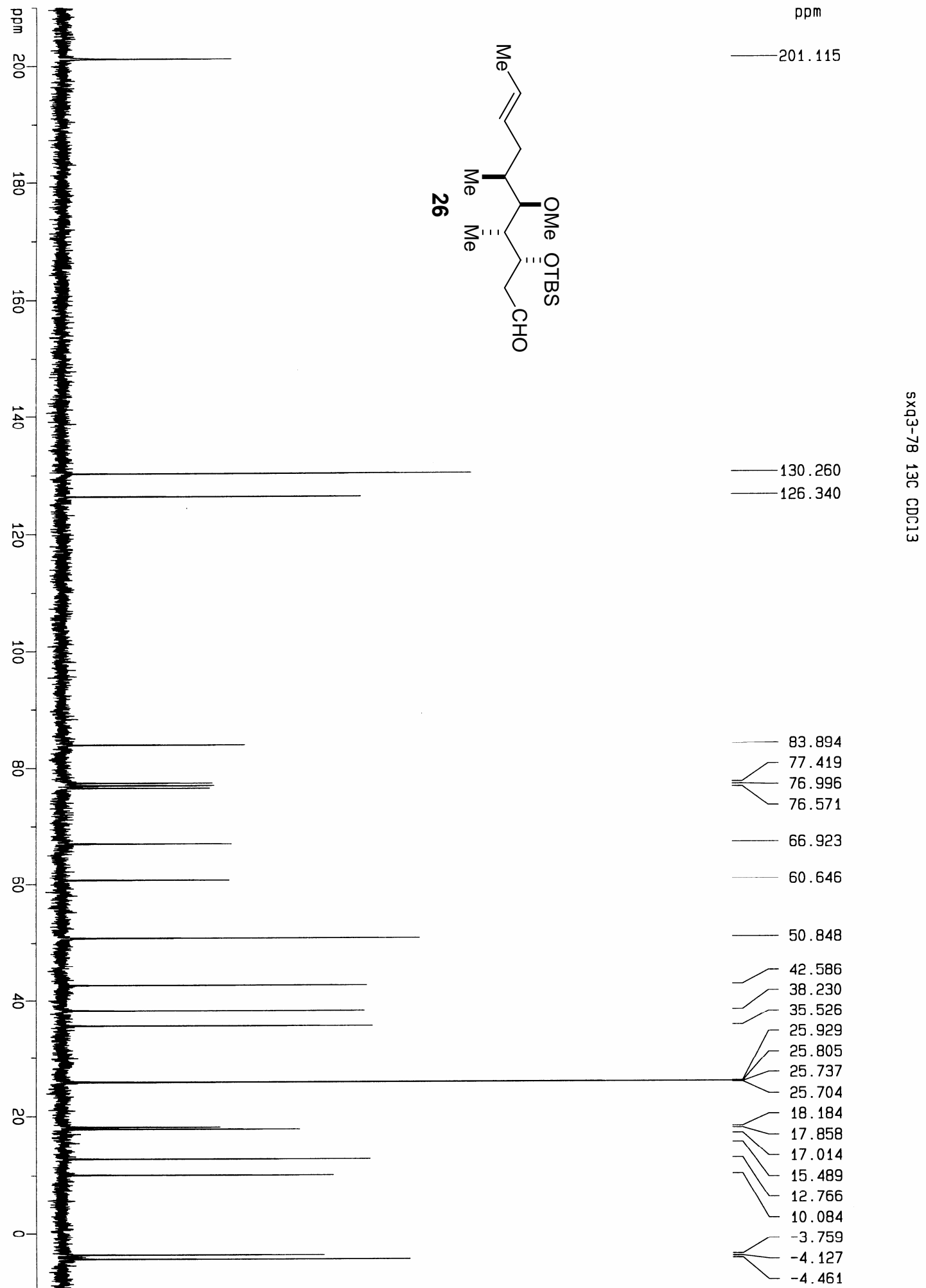

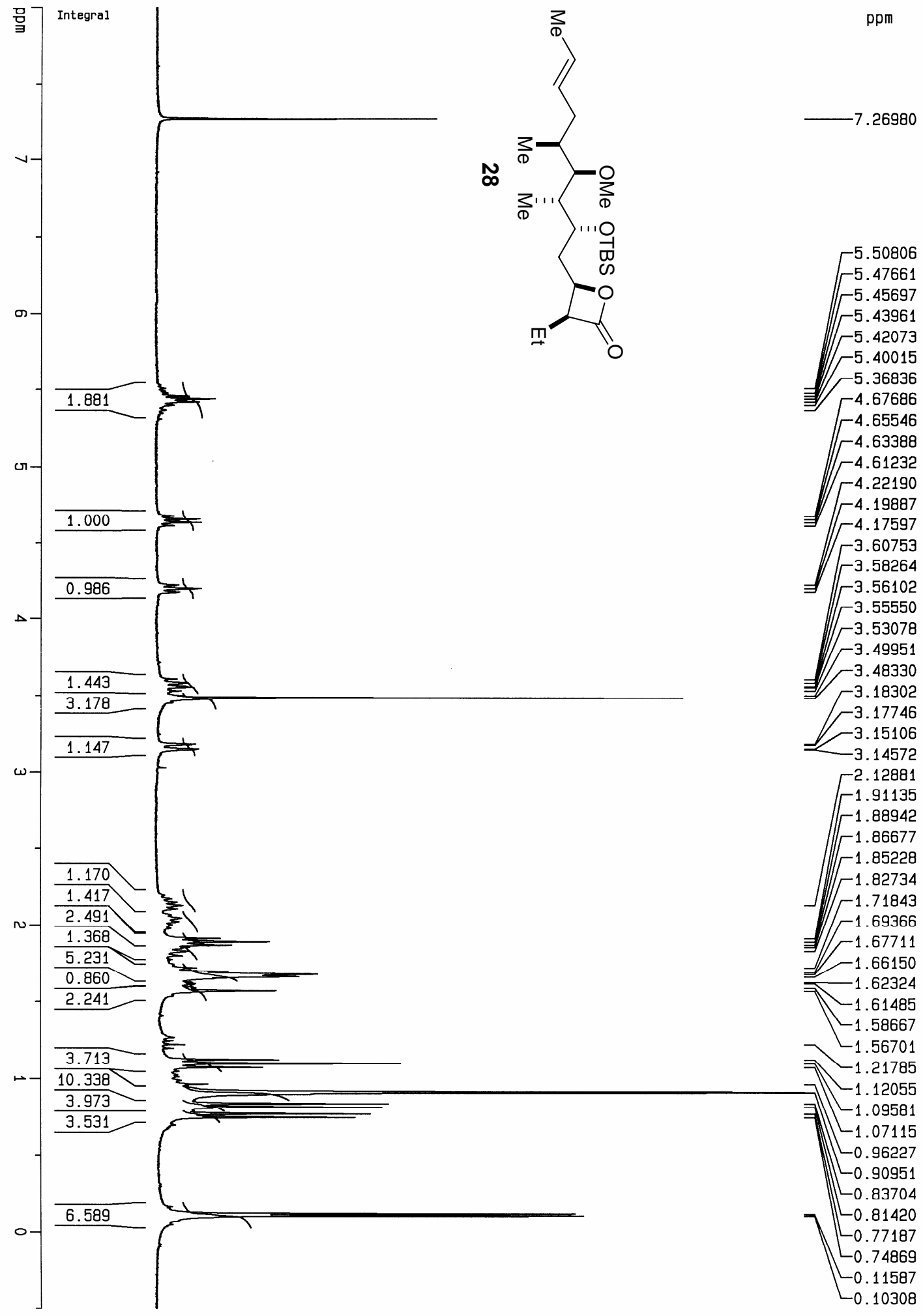

1.91135

$-1.88942$

$\Gamma^{-1.86677}$

$\Gamma^{-1.85228}$

$\Gamma^{1.82734}$

$-1.71843$

$\Gamma^{1.69366}$

$-1.66150$

$-1.62324$

$-1.61485$

$-1.58667$

$-1.56701$

$-1.12055$

$-1.09581$

$-1.07115$

0.90951

0.83704

$\left[\begin{array}{r}0.81420 \\ 0.77187\end{array}\right.$

$-0.74869$

$-0.10308$ 

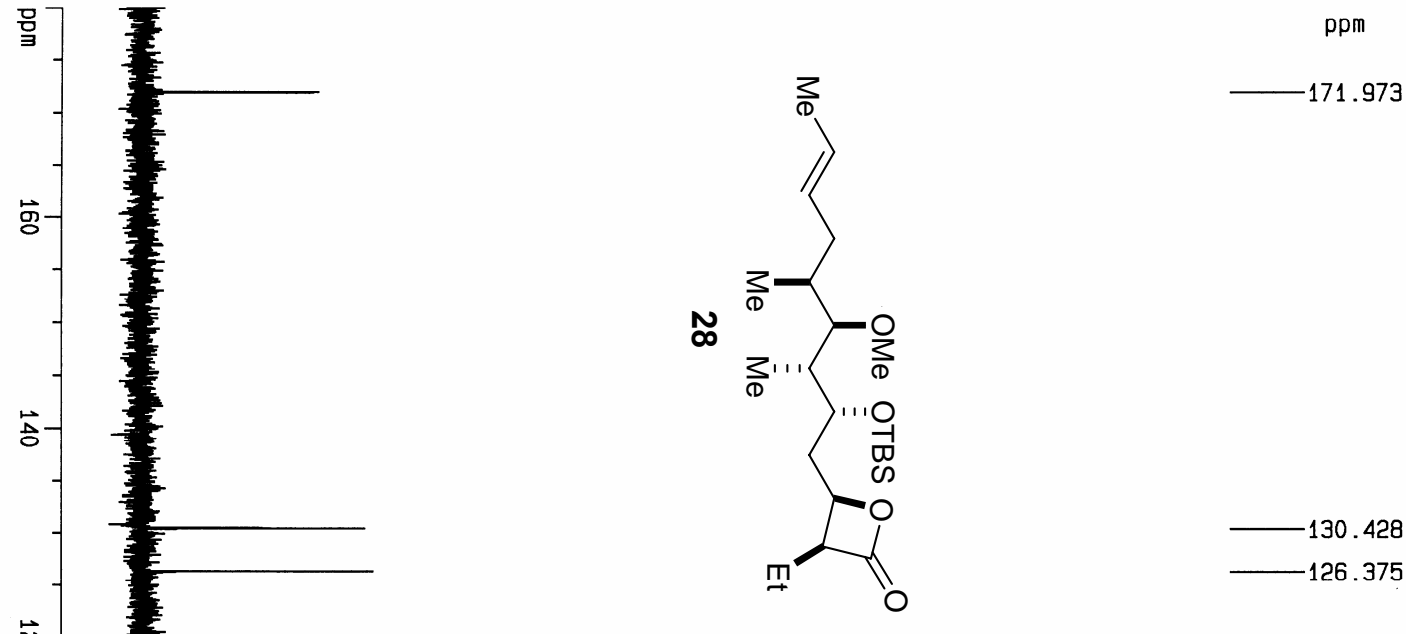

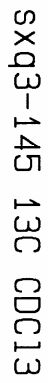

$\overrightarrow{8}$
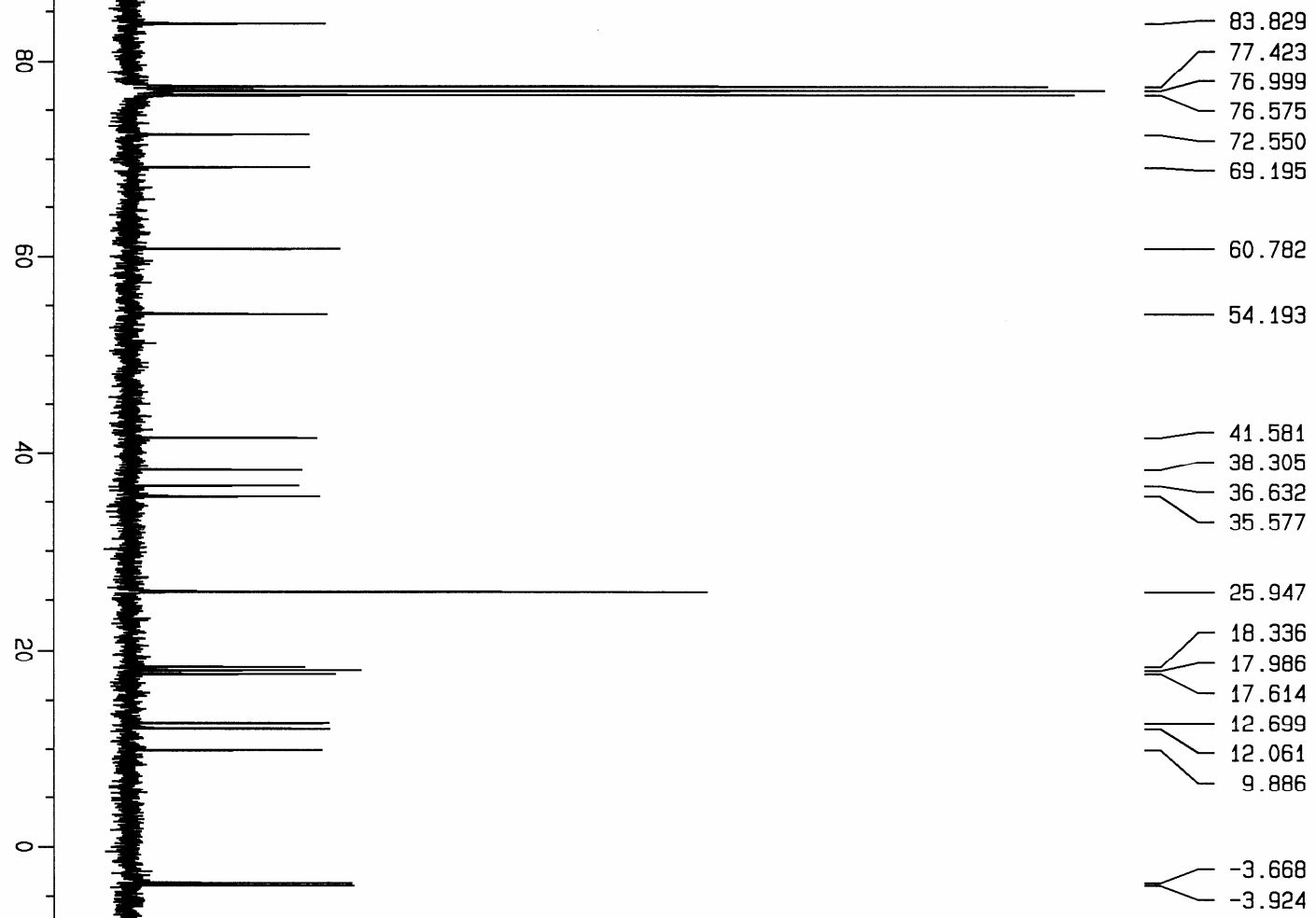


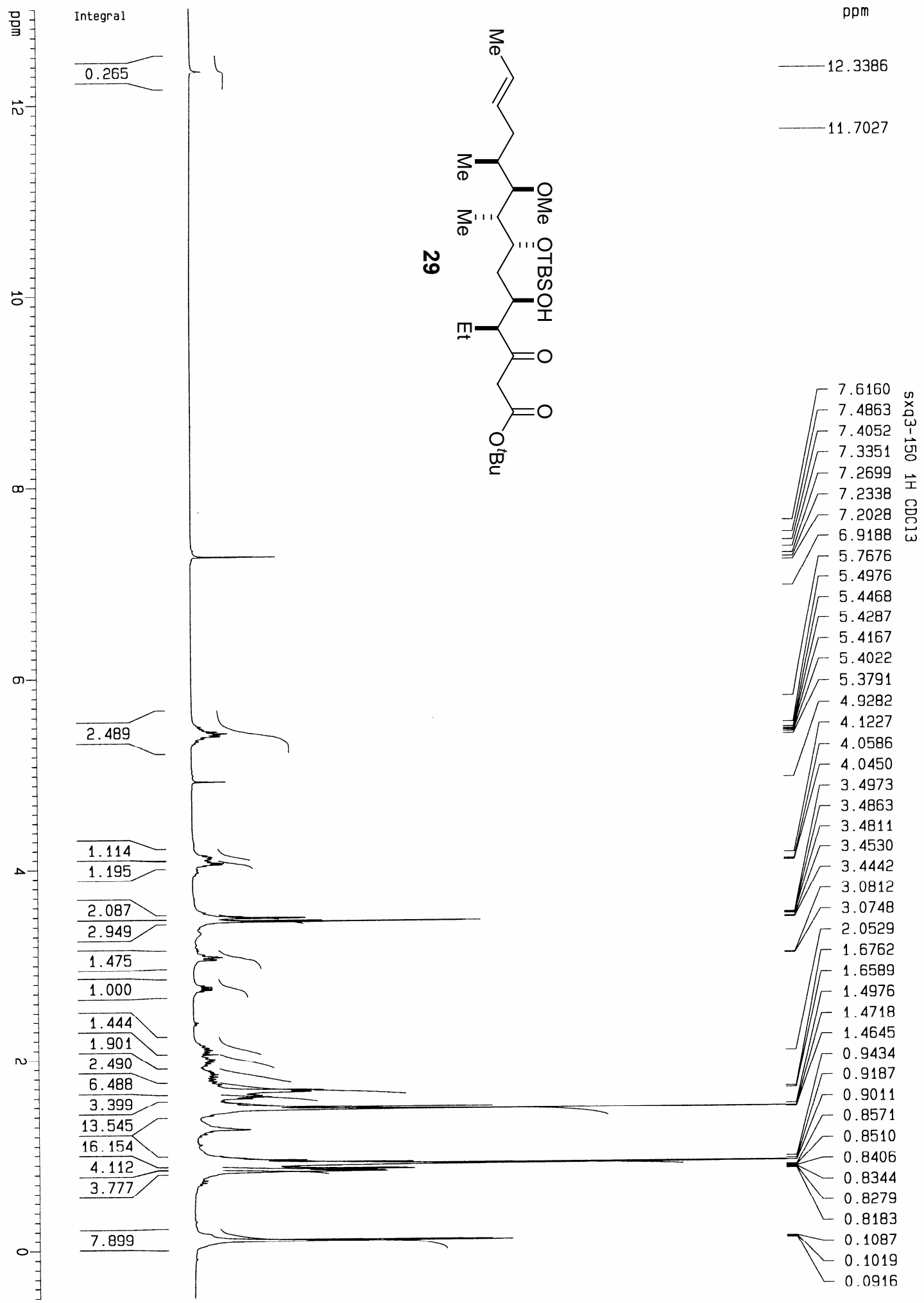




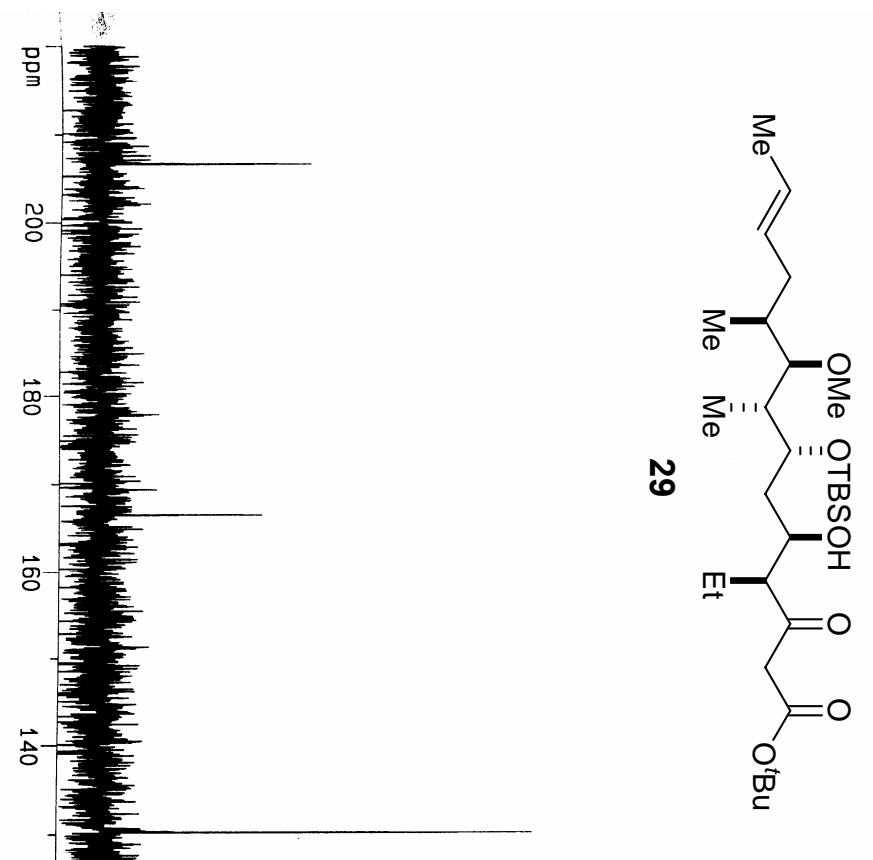

ppm

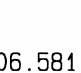

崩

男-
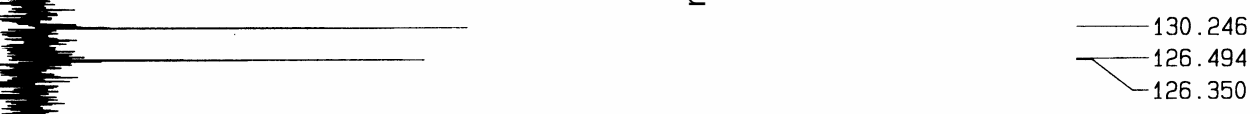

177.872

$-169.363$

$-166.475$

용

of

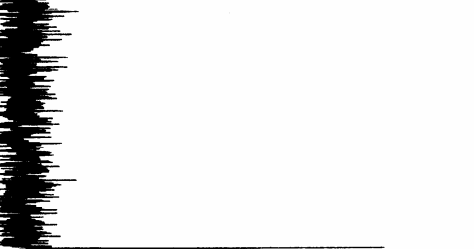

$-81.720$

$-77.424$

77.001

76.579

F 71.560

69.304

- 65.515

- 60.164

F 58.985

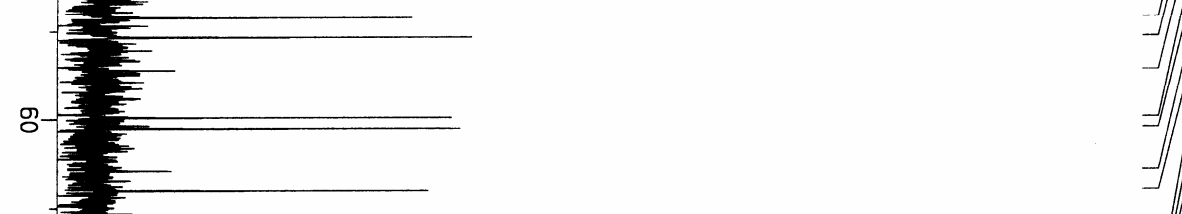

54.265

- 52.006

- 40.713

- 39.169

] 38.593

] 35.506

31.576

$\begin{array}{r}29.685 \\ \hline\end{array}$

- 28.322

- 28.115

- 28.021

$-25.957$

ᄃ 19.805

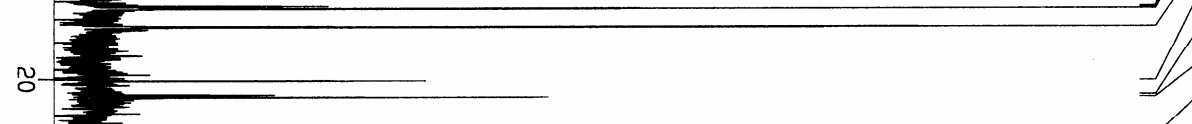

18. 199

17.940

13.398

$-13.240$

$-12.176$

- 12.009

11.475

11.080

$-3.843$

$-4.311$ 

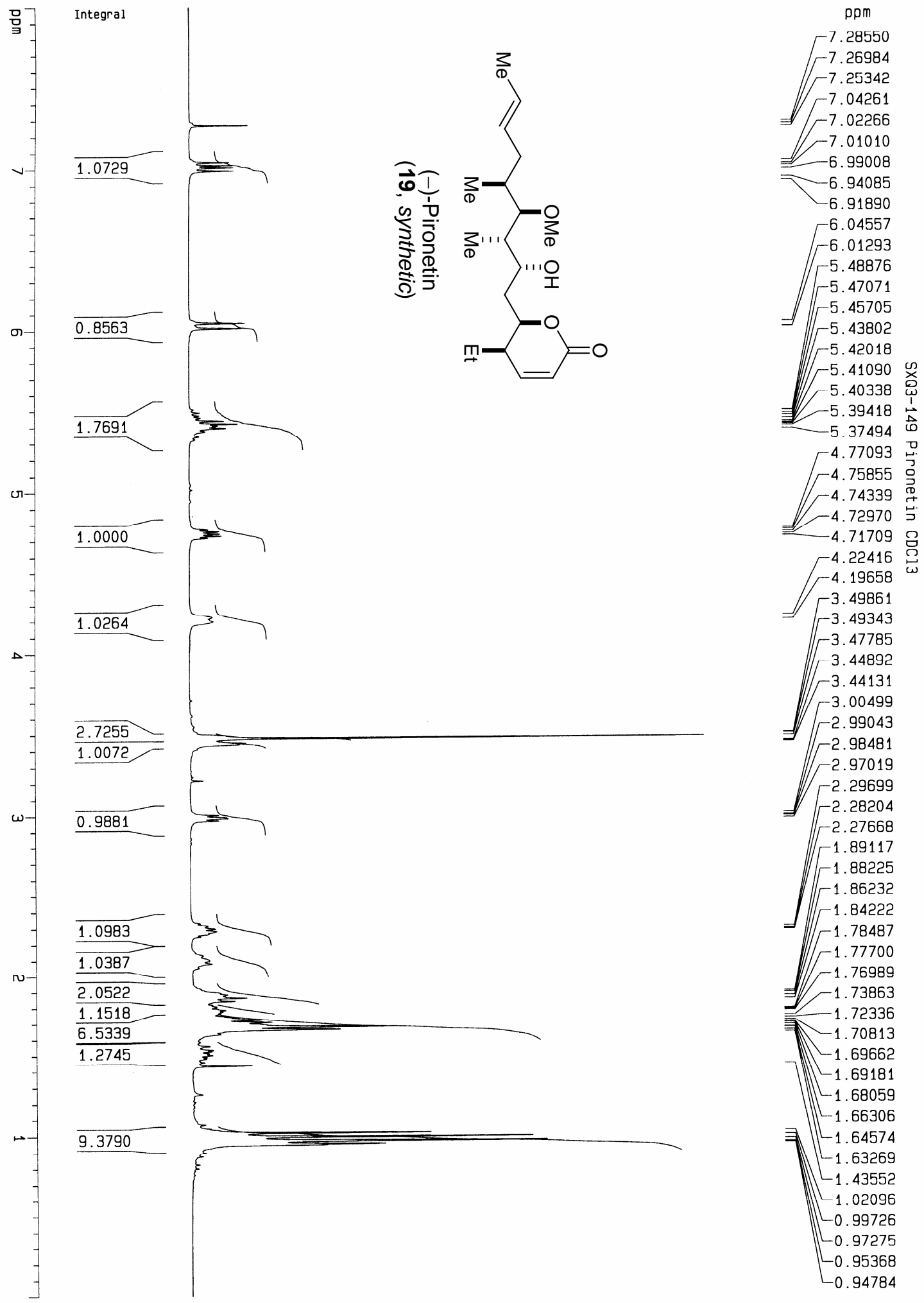


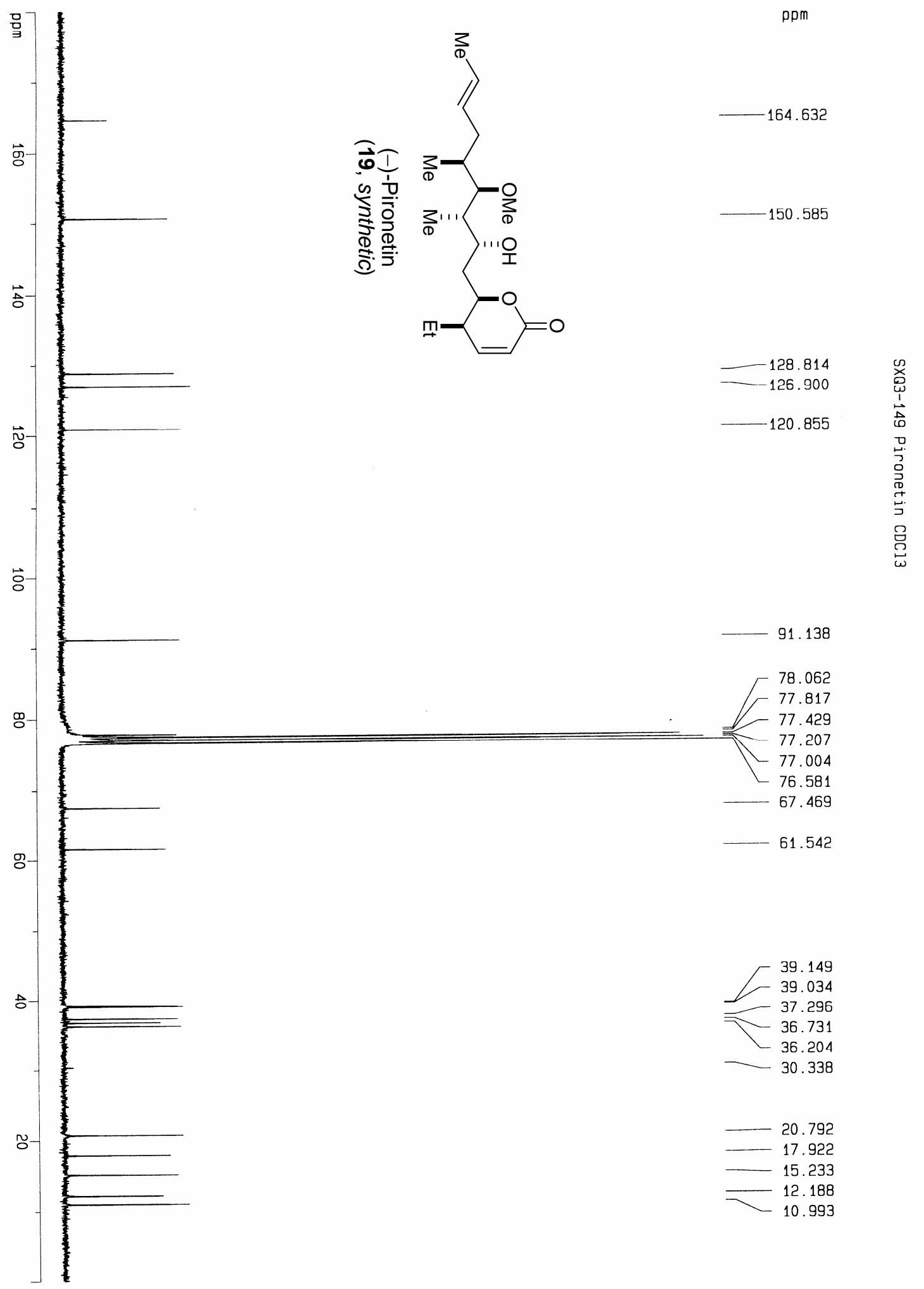

